\title{
A Pauson-Khand Approach to the Synthesis of Ingenol
}

\author{
Jeffrey D. Winkler, Esther C. Y. Lee, and LaToya I. Nevels \\ Department of Chemistry, University of Pennsylvania, Philadelphia, PA 19104 \\ winkler@sas.upenn.edu
}

\section{Supplementary Information}

Table of Contents

Experimental Section

${ }^{1} \mathrm{H}$ NMR Spectrum of 8

${ }^{13} \mathrm{C}$ NMR Spectrum of 8

IR Spectrum of $\mathbf{8}$

${ }^{1} \mathrm{H}$ NMR Spectrum of 9

${ }^{13} \mathrm{C}$ NMR Spectrum of 9

IR Spectrum of 9

${ }^{1} \mathrm{H}$ NMR Spectrum of $\mathbf{1 0}$

${ }^{13} \mathrm{C}$ NMR Spectrum of $\mathbf{1 0}$

IR Spectrum of $\mathbf{1 0}$

${ }^{1} \mathrm{H}$ NMR Spectrum of $\mathbf{1 1}$

${ }^{13} \mathrm{C}$ NMR Spectrum of $\mathbf{1 1}$

IR Spectrum of 11

${ }^{1} \mathrm{H}$ NMR Spectrum of $\mathbf{1 4}$

${ }^{13} \mathrm{C}$ NMR Spectrum of $\mathbf{1 4}$

IR Spectrum of 14

${ }^{1} \mathrm{H}$ NMR Spectrum of $\mathbf{1 5}$

${ }^{13} \mathrm{C}$ NMR Spectrum of $\mathbf{1 5}$

IR Spectrum of $\mathbf{1 5}$

${ }^{1} \mathrm{H}$ NMR Spectrum of $\mathbf{1 6}$

${ }^{13} \mathrm{C}$ NMR Spectrum of $\mathbf{1 6}$
S3-S10

S11

S12

S13

S14

S15

S16

S17

S18

S19

S20

S21

S22

S23

S24

S25

S26

S27

S28

S29

S30 
IR Spectrum of $\mathbf{1 6} \quad$ S31

${ }^{1} \mathrm{H}$ NMR Spectrum of $\mathbf{1 7} \quad$ S32

${ }^{13}$ C NMR Spectrum of $\mathbf{1 7} \quad$ S33

IR Spectrum of $\mathbf{1 7} \quad$ S34

${ }^{1} \mathrm{H}$ NMR Spectrum of $\mathbf{1 8} \quad$ S35

${ }^{13} \mathrm{C}$ NMR Spectrum of $\mathbf{1 8} \quad$ S36

$\begin{array}{ll}\text { IR Spectrum of } \mathbf{1 8} & \text { S37 }\end{array}$

${ }^{1} \mathrm{H}$ NMR Spectrum of $20 \quad$ S38

${ }^{13}$ C NMR Spectrum of $20 \quad$ S39

IR Spectrum of $20 \quad S 40$

${ }^{1} \mathrm{H}$ NMR Spectrum of $\mathbf{2 1}$ S41

${ }^{13} \mathrm{C}$ NMR Spectrum of 21

IR Spectrum of $21 \quad$ S43

${ }^{1} \mathrm{H}$ NMR Spectrum of $22 \quad \mathrm{~S} 44$

${ }^{13} \mathrm{C}$ NMR Spectrum of $22 \quad \mathrm{~S} 45$

IR Spectrum of $22 \quad$ S46

${ }^{1} \mathrm{H}$ NMR Spectrum of $\mathbf{2 3} \quad$ S47

${ }^{13} \mathrm{C}$ NMR Spectrum of $\mathbf{2 3} \quad \mathrm{S} 48$

IR Spectrum of $\mathbf{2 3} \quad$ S49

X-ray Structure Determination of $\mathbf{1 8}$ S50-S57

X-ray Structure Determination of $22 \quad$ S58-S66

X-ray Structure Determination of $\mathbf{2 3} \quad$ S67-75 


\title{
Supporting Information
}

\section{A Pauson-Khand Approach to the Synthesis of Ingenol}

\author{
Jeffrey D. Winkler, Esther C. Y. Lee, and LaToya I. Nevels \\ Department of Chemistry, University of Pennsylvania, Philadelphia, PA 19104
}

\section{EXPERIMENTAL DETAILS}

\section{General Methods}

Solvents used for extraction and purification were HPLC grade from Fisher. Unless otherwise indicated, all reactions were run under an inert atmosphere of Argon. Anhyrous tetrahydrofuran, methylene chloride, benzene, and acetonitrile were obtained via passage through an activated alumina column ${ }^{1}$. Acetonitrile used for photoreactions was taken from the Grubbs system and pre-irradiated for 4 hours with a 450 Watt Hanovia mercury lamp at $0{ }^{\circ} \mathrm{C}$ followed by distillation. Acetone used for photoreactions was freshly distilled from HPLC grade Fisher each time prior to use. A triple-jacketed Pyrex ${ }^{\mathrm{TM}}$ photoreactor was used for photochemical reactions. Commercial reagents were used as received. Deuterated solvents were obtained from Cambridge Isotope Labs. Merck pre-coated silica gel plates $\left(250 \mu \mathrm{M}, 60 \mathrm{~F}_{254}\right)$ were used for analytical TLC. Spots were visualized using $254 \mathrm{~nm}$ ultraviolet light, with either anisaldehyde or potassium permangenate stains as visualizing agents. Chromatographic purifications were performed on Sorbent Technologies silica gel (particle size 32-63 microns). ${ }^{1} \mathrm{H}$ and ${ }^{13} \mathrm{C}$ NMR spectra were recorded on $500 \mathrm{MHz}$ and $125 \mathrm{MHz}$, respectively, in $\mathrm{CDCl}_{3}$ on a Bruker AM-500 or DRX-500 spectrometer. Chemical shifts are reported relative to internal chloroform $\left(\delta 7.26\right.$ for ${ }^{1} \mathrm{H}, \delta 77.0$ for $\left.{ }^{13} \mathrm{C}\right)$. Infrared spectra were recorded on a $\mathrm{NaCl}$ plate using a Perkin-Elmer 1600 series Fourier transform spectrometer. High resolution mass spectra were obtained by Dr. Rakesh Kohli at the University of Pennsylvania Mass Spectrometry Service Center on an Autospec high resolution doublefocusing electrospray ionization/chemical ionization spectrometer with either DEC 11/73 or OPUS software data system. Single-crystal X-ray diffraction structure determination was performed by Dr. Pat Caroll at the University of Pennsylvania. Melting points were obtained on a Thomas Hoover capillary melting point apparatus and are uncorrected.

\footnotetext{
${ }^{1}$ Pangborn, A. B.; Giardello, M. A.; Grubbs, R. H.; Rosen, R. K.; Timmers, F. J. Organometallics 1996, 15, 1518.
} 


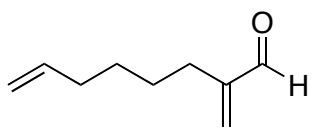

7

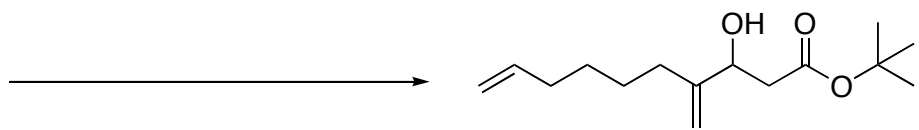

8

$\beta$-hydroxy ester 8. To a solution of diisopropylamine $(5.8 \mathrm{~mL}, 41.2 \mathrm{mmol})$ in anhydrous tetrahydrofuran $(137.0 \mathrm{~mL}, 0.3 \mathrm{M})$ at $-78{ }^{\circ} \mathrm{C}$ was treated with $n$-butyllithium $(17.0 \mathrm{~mL}$, $39.1 \mathrm{mmol}, 2.3 \mathrm{M}$ in hexanes) drop-wise and allowed to stir for $1.2 \mathrm{~h}$. tert-butylacetate $(5.6 \mathrm{~mL}, 41.2 \mathrm{mmol})$ was then added to the reaction mixture neat. The reaction mixture was stirred for $1 \mathrm{~h}$ followed by the addition of $7(2.85 \mathrm{~g}, 20.6 \mathrm{mmol})$ in anhydrous tetrahydrofuran $(10.0 \mathrm{~mL}, 3.0 \mathrm{M})$. The mixture was stirred for $10 \mathrm{~min}$, quenched with saturated $\mathrm{NH}_{4} \mathrm{Cl}$, and extracted with diethyl ether. The combined organic extracts were dried over $\mathrm{MgSO}_{4}$, filtered, and concentrated which afforded $\beta$-hydroxy ester $8(5.1 \mathrm{~g}$, 97\%) as an oil: ${ }^{1} \mathrm{H}$ NMR $\left(500 \mathrm{MHz}, \mathrm{CDCl}_{3}\right) \delta$ 5.83-5.75 (dddd, $J=16.9,10.2,6.6,6.6$ $\mathrm{Hz}, 1 \mathrm{H}), 5.07(\mathrm{~s}, 1 \mathrm{H}), 5.00-4.96(\mathrm{~m}, 1 \mathrm{H}), 4.94-4.91(\mathrm{~m}, 1 \mathrm{H}), 4.86(\mathrm{~s}, 1 \mathrm{H}), 4.42-4.39(\mathrm{~m}$, $1 \mathrm{H}), 3.04(\mathrm{~d}, J=4.1 \mathrm{~Hz}, 1 \mathrm{H}), 2.53-2.49(\mathrm{dd}, J=16.1,3.5 \mathrm{~Hz}, 1 \mathrm{H}), 2.46-2.41(\mathrm{dd}, J=$ $16.1,8.8 \mathrm{~Hz}, 1 \mathrm{H}), 2.12-1.96(\mathrm{~m}, 4 \mathrm{H}), 1.50-1.37(\mathrm{~m}, 13 \mathrm{H}) ;{ }^{13} \mathrm{C} \mathrm{NMR}\left(125 \mathrm{MHz}, \mathrm{CDCl}_{3}\right) \delta$ $172.2,149.8,138.8,114.4,109.9,81.4,70.9,41.3,33.6,31.8,28.7,28.1$, 27.4; FTIR (thin film) 3452, 3077, 2978, 2930, 2858, 2362, 2342, 1730, 1641, 1457, 1393, 1368, 1256, $1151 \mathrm{~cm}^{-1}$. Exact mass calculated for $\mathrm{C}_{15} \mathrm{H}_{23} \mathrm{O}_{3}+\mathrm{Na}$ (CI) 277.1779, found 277.1792 .

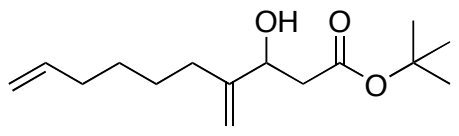

8

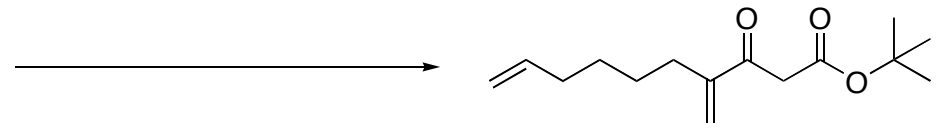

9

$\beta$-ketoester 9. To a flask containing $\beta$-hydroxyester 8 (3.9 $\mathrm{g}, 15.3 \mathrm{mmol})$ was added ethyl acetate $(190.0 \mathrm{~mL}, 0.08 \mathrm{M})$. Mangenese dioxide $(73.2 \mathrm{~g}, 841.0 \mathrm{mmol})$ was added as a solid over 3 days. The reaction was filtered through celite and concentrated to afford $\beta$-ketoester 9 (3.3 g, 86\%) as an oil: ${ }^{1} \mathrm{H}$ NMR $\left(500 \mathrm{MHz}, \mathrm{CDCl}_{3}\right) \delta 5.97(\mathrm{~s}, 1 \mathrm{H}), 5.82(\mathrm{~s}$, $1 \mathrm{H}), 5.80(\mathrm{~m}, 1 \mathrm{H}), 4.99(\mathrm{dd}, J=17.1,1.9 \mathrm{~Hz}, 1 \mathrm{H}), 4.93(\mathrm{dd}, J=10.2,2.0 \mathrm{~Hz}, 1 \mathrm{H}), 3.61$ $(\mathrm{s}, 2 \mathrm{H}), 2.28(\mathrm{t}, J=6.5 \mathrm{~Hz}, 2 \mathrm{H}), 2.05(\mathrm{q}, J=7.0 \mathrm{~Hz}, 2 \mathrm{H}), 1.45(\mathrm{~s}, 9 \mathrm{H}), 1.40(\mathrm{~m}, 4 \mathrm{H}) ;{ }^{13} \mathrm{C}$ NMR $\left(125 \mathrm{MHz}, \mathrm{CDCl}_{3}\right) \delta 194.3,166.9,148.6,138.7,125.4,114.4,81.8,46.8,33.5$, 30.5, 28.6, 27.9(3), 27.8; FTIR (thin film) 3076, 2978, 2930, 2859, 1736, 1682, 1640, $1590,1456,1410,1367,1305,1256,1150,1074,995,955,911,339 \mathrm{~cm}^{-1}$. Exact mass calculated for $\mathrm{C}_{15} \mathrm{H}_{24} \mathrm{O}_{3}+\mathrm{H}(\mathrm{CI})$ 253.1803, found 253.1816. 


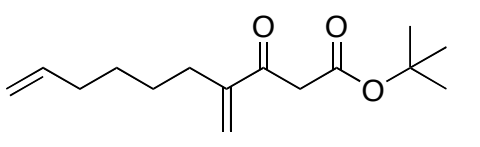

9

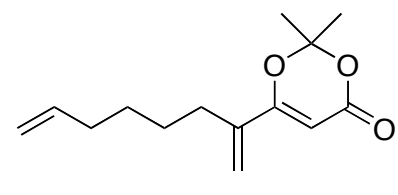

10

Dioxenone 10. A solution of $\beta$-ketoester $9(3.0 \mathrm{~g}, 11.9 \mathrm{mmol})$ in freshly distilled acetone $(52.0 \mathrm{~mL}, 0.23 \mathrm{M})$ was cooled to $-78{ }^{\circ} \mathrm{C}$. Trifluoroacetic anhydride $(10.4 \mathrm{~mL}, 1.14 \mathrm{M})$, trifluoroacetic acid $(52.0 \mathrm{~mL}, 0.23 \mathrm{M})$, and acetic anhydride $(8.1 \mathrm{~mL}, 1.47 \mathrm{M})$ were added dropwise, in that order. The solution was stirred for $12 \mathrm{~h}$, gradually warming to room temperature. The reaction was slowly added to a cold saturated solution of $\mathrm{NaHCO}_{3}$ and extracted with ethyl acetate. The combined organic layers were dried over $\mathrm{MgSO}_{4}$, filtered, concentrated, and purified by silica gel chromatography $(10 \%$ ether/hexane) to yield Dioxenone $10(2.1 \mathrm{~g}, 75 \%)$ as a yellow oil: ${ }^{1} \mathrm{H}$ NMR (500 $\mathrm{MHz}$, $\left.\mathrm{CDCl}_{3}\right) \delta 5.80-5.72(\mathrm{~m}, 1 \mathrm{H}), 5.79(\mathrm{~s}, 1 \mathrm{H}), 5.48(\mathrm{~s}, 1 \mathrm{H}), 5.34(\mathrm{~s}, 1 \mathrm{H}), 5.00-4.96(\mathrm{ddt}, J=$ $17.2,1.7,1.5 \mathrm{~Hz}, 1 \mathrm{H}), 4.95-4.92(\mathrm{~m}, 1 \mathrm{H}), 2.23-2.20(\mathrm{t}, J=7.4 \mathrm{~Hz}, 2 \mathrm{H}), 2.13-2.01(\mathrm{~m}$, 2H), 1.68 (s, 6H), 1.57-1.37 (m, 4H); $\left.{ }^{13} \mathrm{C} \mathrm{NMR} \mathrm{(125} \mathrm{MHz,} \mathrm{CDCl}_{3}\right) \delta 164.5,162.0,140.2$, 138.4, 120.2, 114.7, 106.2, 92.6, 33.4, 31.4, 28.4, 27.8, 24.9; FTIR (thin film) 2298, 2931, 2858, 1729, 1632, 1591, 1461, 1439, 1390, 1376, 1348, 1281, 1253, 1205, 1004 $\mathrm{cm}^{-1}$. Exact mass calculated for $\mathrm{C}_{14} \mathrm{H}_{20} \mathrm{O}_{3}+\mathrm{Na}(\mathrm{CI}) 259.1310$, found 259.1315.

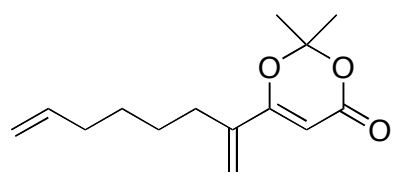

10

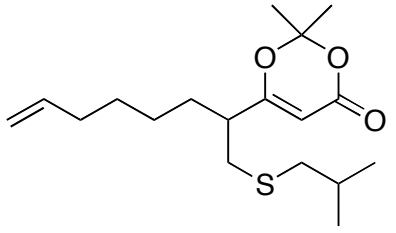

14

Sulfide Dioxenone 14. To a flame-dried flask containing dioxenone 10 (5.3 g, 22.4 $\mathrm{mmol})$ and dry dichloromethane $(450.0 \mathrm{~mL}, 0.05 \mathrm{M})$ was added triethylamine $(37.5 \mathrm{~mL}$, $269.0 \mathrm{mmol})$ and 2-methyl-1-propane thiol $(13.6 \mathrm{~mL}, 125.4 \mathrm{mmol})$ at room temperature. The reaction was stirred under argon at room temperature for $24 \mathrm{~h}$. The reaction was quenched with pH 7 buffer and extracted with methylene chloride. The combined organics were washed with brine, dried over $\mathrm{MgSO}_{4}$, filtered, and concentrated. Purification by silica gel chromatography (10\% ether/hexane) afforded sulfide 14 (7.2 g, $98 \%$ ) as an oil: ${ }^{1} \mathrm{H}$ NMR $\left(500 \mathrm{MHz}, \mathrm{CDCl}_{3}\right) \delta 5.76$ (dddd, $J=16.9,10.2,6.7,6.7 \mathrm{~Hz}$, $1 \mathrm{H}), 5.26(\mathrm{~s}, 1 \mathrm{H}), 4.98(\mathrm{dd}, J=17.1,1.9 \mathrm{~Hz}, 1 \mathrm{H}), 4.93(\mathrm{dd}, J=10.2,2.0 \mathrm{~Hz}, 1 \mathrm{H}), 2.64$ $(\mathrm{dd}, J=12.9,8.7 \mathrm{~Hz}, 1 \mathrm{H}), 2.55(\mathrm{dd}, J=12.9,5.9 \mathrm{~Hz}, 1 \mathrm{H}), 2.37(\mathrm{~d}, J=6.9 \mathrm{~Hz}, 2 \mathrm{H}), 2.34$ (m, 1H), $2.02(\mathrm{ddd}, J=6.8,6.8,6.8 \mathrm{~Hz}, 2 \mathrm{H}), 1.74(\mathrm{~m}, 1 \mathrm{H}), 1.69(\mathrm{~s}, 3 \mathrm{H}), 1.67(\mathrm{~s}, 3 \mathrm{H})$, $1.55(\mathrm{~m}, 2 \mathrm{H}), 1.37(\mathrm{~m}, 2 \mathrm{H}), 1.30(\mathrm{~m}, 2 \mathrm{H}), 0.96(\mathrm{~d}, J=6.6 \mathrm{~Hz}, 6 \mathrm{H}) ;{ }^{13} \mathrm{C}$ NMR $(125 \mathrm{MHz}$, $\left.\mathrm{CDCl}_{3}\right) \delta 172.2,161.1,138.4,114.6,106.5,94.5,44.7,42.0,34.9,33.4,31.4,28.5,28.5$, 26.5, 25.4, 24.8, 22.0, 21.9; FTIR (thin film) 3075, 2930, 2864, 1734, 1633, 1461, 1384, $1271,1250,1204,1014,902,857,810 \mathrm{~cm}^{-1}$. Exact mass calculated for $\mathrm{C}_{18} \mathrm{H}_{30} \mathrm{O}_{3} \mathrm{~S}+\mathrm{Na}$ (ES) 349.1814, found 349.1801. 


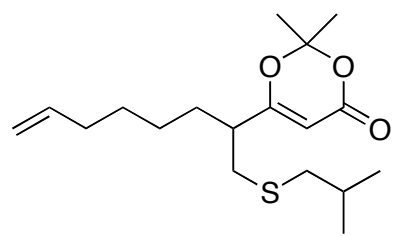

14

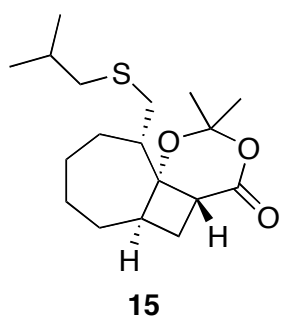

15

Sulfide photoadduct 15. In a $60 \mathrm{~mL}$ oven-dried Pyrex ${ }^{\mathrm{TM}}$ photoreactor, was added sulfide $14(60 \mathrm{mg}, 0.18 \mathrm{mmol})$ in pre-irradiated and distilled acetonitrile $(54 \mathrm{~mL}, 3.3 \mathrm{M})$ and freshly distilled acetone $(6.0 \mathrm{~mL}, 0.3 \mathrm{M})$. The mixture was purged with argon at $0{ }^{\circ} \mathrm{C}$ for $1 \mathrm{~h}$. Using a $450 \mathrm{~W}$ Mercury medium pressure lamp, the reaction was irradiated for 60 min at $0{ }^{\circ} \mathrm{C}$. The reaction was concentrated and purified by silica gel chromatography (1\% acetone/hexane) to afford sulfoxide $15(19 \mathrm{mg}, 31 \%)$ as an oil: ${ }^{1} \mathrm{H}$ NMR $(500 \mathrm{MHz}$, $\left.\mathrm{CDCl}_{3}\right) \delta 3.02(\mathrm{dd}, J=11.5,5.2 \mathrm{~Hz}, 1 \mathrm{H}), 2.69(\mathrm{~m}, 1 \mathrm{H}), 2.42(\mathrm{~m}, 1 \mathrm{H}), 2.37(\mathrm{dd}, J=6.6$, $3.9 \mathrm{~Hz}, 2 \mathrm{H}), 2.30(\mathrm{~m}, 1 \mathrm{H}), 2.26(\mathrm{~m}, 1 \mathrm{H}), 1.93$ (ddd, $J=11.9,11.9,8.0 \mathrm{~Hz}, 1 \mathrm{H}), 1.83(\mathrm{~m}$, $4 \mathrm{H}), 1.77(\mathrm{~m}, 1 \mathrm{H}), 1.74(\mathrm{~s}, 3 \mathrm{H}), 1.52(\mathrm{~s}, 3 \mathrm{H}), 1.32(\mathrm{~m}, 5 \mathrm{H}), 0.99(\mathrm{~d}, J=6.6 \mathrm{~Hz}, 6 \mathrm{H}) ;{ }^{13} \mathrm{C}$ NMR $\left(125 \mathrm{MHz} \mathrm{CDCl}_{3}\right) \delta 171.7,106.0,82.3,46.8,45.3,42.2,36.2$, 34.6, 33.3, 30.7, 30.0, 29.5, 28.7, 28.6, 27.5, 25.9, 22.2, 22.0; FTIR (thin film) 2924, 2854, 1741, 1460, $1384,1374,1308,1276,1200,1143,1092,1018,1001,988,930 \mathrm{~cm}^{-1}$. Exact mass calculated for $\mathrm{C}_{18} \mathrm{H}_{30} \mathrm{O}_{3} \mathrm{~S}+\mathrm{Na}(\mathrm{ES}) 349.1814$, found 349.1801 .

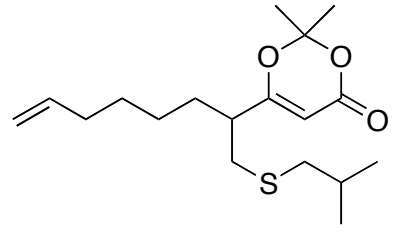

14

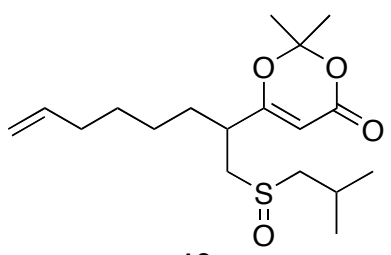

16

Sulfoxide Dioxenone 16. To a flask containing sulfide dioxenone 14 (1.26 g, $3.8 \mathrm{mmol})$ and dry methylene chloride $(140.0 \mathrm{~mL}, 0.025 \mathrm{M})$ at $-78{ }^{\circ} \mathrm{C}$ was added a solution of $68 \%$ $m$-CPBA $(1.1 \mathrm{~g}, 4.2 \mathrm{mmol})$ in dry methylene chloride $(40.0 \mathrm{~mL}, 0.1 \mathrm{M})$. The reaction was stirred for $2 \mathrm{~h}$ at $-78{ }^{\circ} \mathrm{C}$. The reaction was quenched with saturated $\mathrm{NaHCO}_{3}$ solution and extracted with methylene chloride. The combined organics were washed with saturated $\mathrm{NaHCO}_{3}$, dried over $\mathrm{MgSO}_{4}$, filtered, and concentrated. Purification by silica gel chromatography (5-10\% ethyl acetate/hexane) afforded sulfoxide 16 (1.26 g, 97\%) as an oil: ${ }^{1} \mathrm{H}$ NMR (500 MHz, $\left.\mathrm{CDCl}_{3}\right) \delta 5.70(\mathrm{~m}, 2 \mathrm{H}), 5.36$ (s, 1H, major), 5.27 (s, $1 \mathrm{H}$, minor), $4.94(\mathrm{~d}, J=17.1 \mathrm{~Hz}, 2 \mathrm{H}), 4.90(\mathrm{~d}, J=10.1 \mathrm{~Hz}, 2 \mathrm{H}), 2.87-2.60(\mathrm{~m}, 8 \mathrm{H}), 2.35$ $(\mathrm{m}, 2 \mathrm{H}), 2.17(\mathrm{~m}, 2 \mathrm{H}), 1.98(\mathrm{~m}, 4 \mathrm{H}), 1.76-1.50(\mathrm{~m}, 16 \mathrm{H}), 1.34(\mathrm{~m}, 8 \mathrm{H}), 1.03(\mathrm{~m}, 12 \mathrm{H})$; ${ }^{13} \mathrm{C} \mathrm{NMR}\left(125 \mathrm{MHz}, \mathrm{CDCl}_{3}\right) \delta 170.7,170.0,160.5,160.3,138.1,138.1,114.7$ (2), 106.6, 106.6, 95.3, 94.2, 62.8, 62.4, 55.3, 55.2, 38.9, 38.5, 33.1 (2), 32.1, 30.5, 28.3, 26.1, 26.0, 25.2, 25.0, 24.9, 24.8, 23.9, 23.8, 22.7, 22.6, 21.6, 21.5; FTIR (thin film) 3435, 3076, 2994, 2958, 2933, 2867, 1730, 1633, 1462, 1387, 1272, 1204, 1075, 1021, 903, 856, 813 $\mathrm{cm}^{-1}$. Exact mass calculated for $\mathrm{C}_{18} \mathrm{H}_{30} \mathrm{O}_{4} \mathrm{~S}+\mathrm{Na}(\mathrm{ES}) 365.1762$, found 365.1745 . 


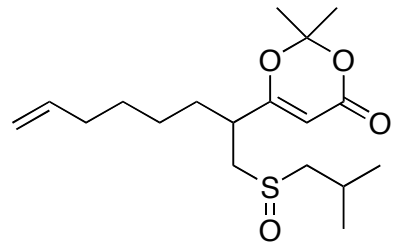

16

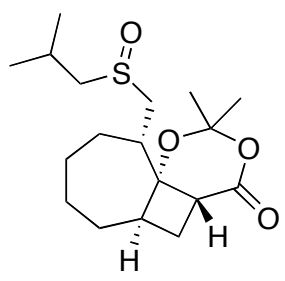

17

Sulfoxide photoadduct 17. In an oven-dried 1L quartz photoreactor with Pyrex ${ }^{\mathrm{TM}}$ insert was added a solution of sulfoxide $16(1.0 \mathrm{~g}, 2.9 \mathrm{mmol})$, in pre-irradiated and distilled acetonitrile $(261.0 \mathrm{~mL}, 11.1 \mathrm{mM})$, and freshly distilled acetone $(29.0 \mathrm{~mL}, 100.0 \mathrm{mM})$. The solution was purged with argon at $0{ }^{\circ} \mathrm{C}$ for $1 \mathrm{~h}$. Using a $450 \mathrm{~W}$ Mercury medium pressure lamp, the reaction was irradiated for $75 \mathrm{~min}$ at $0{ }^{\circ} \mathrm{C}$. The reaction was concentrated to afford sulfoxide cycloadduct $17(1.0 \mathrm{~g}, 100 \%)$ as an oil: ${ }^{1} \mathrm{H}$ NMR (500 $\left.\mathrm{MHz} \mathrm{CDCl}_{3}\right) \delta 3.05$ (dd, $J=11.7,5.5 \mathrm{~Hz}, 1 \mathrm{H}$, major), $3.00(11.7,5.5 \mathrm{~Hz}, 1 \mathrm{H}$, minor), $2.71(\mathrm{~m}, 2 \mathrm{H}), 2.65(\mathrm{dd}, J=12.8,5.0 \mathrm{~Hz}, 2 \mathrm{H}$, minor), $2.61(\mathrm{dd}, J=12.7,4.9 \mathrm{~Hz}, 2 \mathrm{H}$, major), $2.54(\mathrm{~d}, J=6.2 \mathrm{~Hz}, 2 \mathrm{H}), 2.35-2.28(\mathrm{~m}, 5 \mathrm{H}), 2.22(\mathrm{~m}, 2 \mathrm{H}), 2.15(\mathrm{~m}, 2 \mathrm{H}), 1.96(\mathrm{~m}$, $2 \mathrm{H}), 1.82(\mathrm{~m}, 1 \mathrm{H}), 1.75(\mathrm{~m}, 8 \mathrm{H}), 1.71(\mathrm{~m}, 6 \mathrm{H}), 1.54(\mathrm{~m}, 1 \mathrm{H}), 1.51(\mathrm{~s}, 3 \mathrm{H}), 1.48(\mathrm{~s}, 3 \mathrm{H})$, $1.42(\mathrm{~m}, 4 \mathrm{H}), 1.22(\mathrm{~m}, 3 \mathrm{H}), 1.05(\mathrm{~m}, 6 \mathrm{H}), 1.01(\mathrm{~m}, 4 \mathrm{H}) ;{ }^{13} \mathrm{C} \mathrm{NMR}\left(125 \mathrm{MHz}, \mathrm{CDCl}_{3}\right)$ $\delta 170.9,170.8,106.0,105.8,82.6,82.6,62.2$, 62.5, 55.4, 46.1 (2), 40.9, 39.4, 35.9, 35.5, $32.9,32.5,30.5,30.4,30.4,29.3,29.2,29.1,28.8,28.4,27.3,27.1,25.1,25.0,23.9,23.8$, 22.7, 22.6, 21.6, 21.5; FTIR (thin film) 3425, 2954, 2926, 2868, 1738, 1459, 1380, 1308, $1278,1200,1142,1097,1072,1024,931,884,785,731 \mathrm{~cm}^{-1}$. Exact mass calculated for $\mathrm{C}_{18} \mathrm{H}_{30} \mathrm{O}_{4} \mathrm{~S}+\mathrm{Na}(\mathrm{ES}) 365.1762$, found 365.1772.

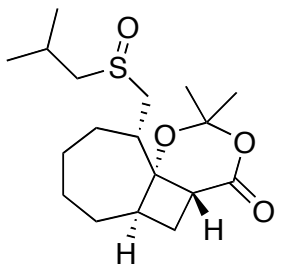

17

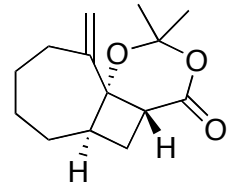

11

Unsaturated photoadduct 11. In an oven-dried flask, containing sulfoxide cylcoadduct 17 (25 mg, $0.073 \mathrm{mmol}$ ) was added quinoline $(1.0 \mathrm{~mL}, 0.07 \mathrm{M})$ at room temperature. The reaction was heated to $1600^{\circ} \mathrm{C}$ for $6 \mathrm{~h}$. The reaction was quenched with $\mathrm{pH} 7$ buffer and extracted with methylene chloride. The combined organic extracts were dried with $\mathrm{MgSO}_{4}$, filtered, and concentrated. Purification by silica gel chromatography (5\% ethyl acetate/hexane) afforded unsaturated cycloadduct $11(10 \mathrm{mg}, 53 \%)$ as a white solid: $\mathrm{mp}$ 74-76 ${ }^{\circ} \mathrm{C} ;{ }^{1} \mathrm{H}$ NMR $\left(500 \mathrm{MHz}, \mathrm{CDCl}_{3}\right) \delta 5.10(\mathrm{~s}, 1 \mathrm{H}), 4.99(\mathrm{~s}, 1 \mathrm{H}), 3.36-3.33$ (dd, $J=$ $11.1,3.1 \mathrm{~Hz}, 1 \mathrm{H}), 2.48-2.38(\mathrm{~m}, 3 \mathrm{H}), 2.29-2.25(\mathrm{~m}, 1 \mathrm{H}), 1.88-1.82(\mathrm{~m}, 1 \mathrm{H}), 1.82-1.66$ $(\mathrm{m}, 3 \mathrm{H}), 1.55(\mathrm{~s}, 3 \mathrm{H}), 1.41(\mathrm{~s}, 3 \mathrm{H}), 1.31-1.19(\mathrm{~m}, 2 \mathrm{H}), 1.17-1.08(\mathrm{~m}, 1 \mathrm{H}) ;{ }^{13} \mathrm{C} \mathrm{NMR}(125$ $\left.\mathrm{MHz} \mathrm{CDCl}_{3}\right) \delta 172.4,149.8,110.8,107.2,82.1,45.8,33.5,32.5,32.4,31.6,29.5,27.3$, 26.9, 25.9; FTIR (thin film) 2295, 2923, 2852, 1740, 1642, 1450, 1378, 1316, 1289, $1242,1184,1131,1097,1070,1036 \mathrm{~cm}^{-1}$. Exact mass calculated for $\mathrm{C}_{14} \mathrm{H}_{30} \mathrm{O}_{3}+\mathrm{H}(\mathrm{CI})$ 237.1491 , found 237.1502 . 

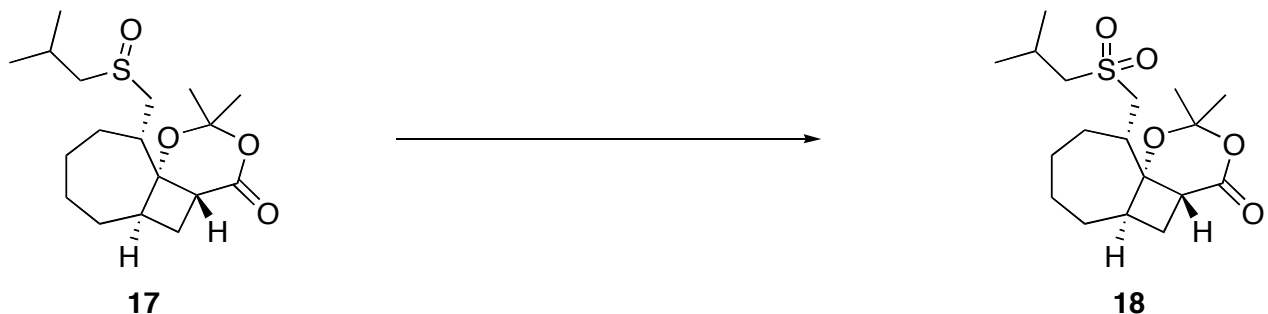

Sulfone photoadduct 18. To a solution of sulfoxide $17(67.0 \mathrm{mg}, 0.19 \mathrm{mmol})$ in dry methylene chloride $(10.0 \mathrm{~mL}, 0.02 \mathrm{M})$ at room temperature was added a solution of $\mathrm{m}$ CPBA (149.0 mg, $0.59 \mathrm{mmol}, 68 \%)$ in dry methylene chloride $(1.0 \mathrm{~mL})$. The reaction was stirred at room temperature for $30 \mathrm{~min}$. The reaction was quenched with saturated $\mathrm{NaHCO}_{3}$ and extracted with methylene chloride. The combined organic layers were dried with $\mathrm{MgSO}_{4}$, filtered, and concentrated. Purification by silica gel chromatography (10\% ethyl acetate/hexane) afforded sulfone $\mathbf{1 8}(51 \mathrm{mg}, 72 \%)$ as a crystalline solide: $\mathrm{mp}$ $109^{\circ}-112^{\circ} \mathrm{C}$; ${ }^{1} \mathrm{H}$ NMR $\left(500 \mathrm{MHz}, \mathrm{CDCl}_{3}\right) \delta 3.02(\mathrm{dd}, J=5.6,11.9 \mathrm{~Hz}, 1 \mathrm{H}), 2.85(\mathrm{~d}, J=$ $6.6 \mathrm{~Hz}, 2 \mathrm{H}), 2.82(\mathrm{~m}, 1 \mathrm{H}), 2.75(\mathrm{~m}, 1 \mathrm{H}), 2.63(\mathrm{~d}, J=13.8 \mathrm{~Hz}, 1 \mathrm{H}), 2.52(\mathrm{t}, J=9.4 \mathrm{~Hz}$, $1 \mathrm{H}), 2.33$ (dddd, $J=13.4,13.4,6.7,6.7 \mathrm{~Hz}, 1 \mathrm{H}), 2.25$ (ddd, $J=13.0,10.4,5.7 \mathrm{~Hz}, 1 \mathrm{H}$ ), $2.00(\mathrm{~m}, 1 \mathrm{H}), 1.95(\mathrm{~m}, 1 \mathrm{H}), 1.88(\mathrm{~m}, 1 \mathrm{H}), 1.79(\mathrm{~m}, 3 \mathrm{H}), 1.75(\mathrm{~s}, 3 \mathrm{H}), 1.52(\mathrm{~s}, 3 \mathrm{H}), 1.45$ $(\mathrm{m}, 2 \mathrm{H}), 1.24(\mathrm{~m}, 1 \mathrm{H}), 1.09(\mathrm{~d}, J=6.7 \mathrm{~Hz}, 6 \mathrm{H}) ;{ }^{13} \mathrm{C} \mathrm{NMR}\left(125 \mathrm{MHz}, \mathrm{CDCl}_{3}\right) \delta 170.7$, 106.1, 83.0, 61.8, 54.8, 45.6, 39.4, 35.5, 32.9, 30.5, 30.3, 29.3, 29.0, 27.4, 24.7, 23.6, 22.7, 22.7; FTIR (thin film) 2929, 2872, 2362, 2254, 1737, 1459, 1384, 1296, 1203, $1135,1110,1019,1004,931,888,829,784,733 \mathrm{~cm}^{-1}$. Exact mass calculated for $\mathrm{C}_{18} \mathrm{H}_{30} \mathrm{O}_{5} \mathrm{~S}+\mathrm{Na}$ (ES) 381.1711, found 381.1721.
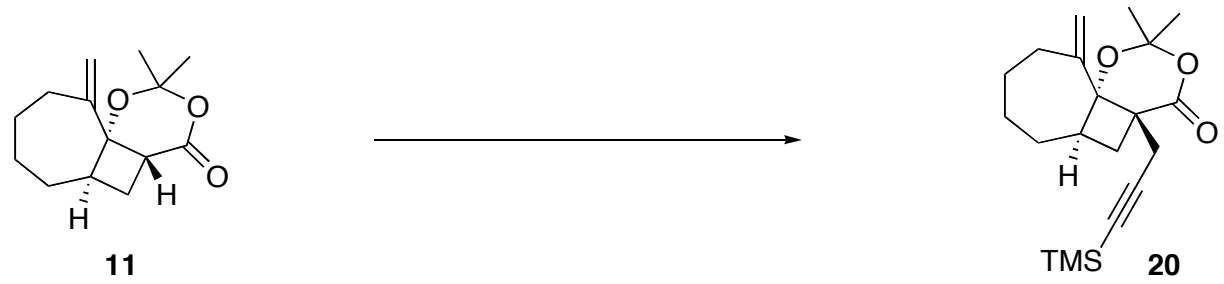

Alkyne 20. $n$-BuLi (1.8 mL of a $2.36 \mathrm{M}$ solution in hexanes, $4.2 \mathrm{mmol})$ was added to freshly distilled diisopropylamine $(0.63 \mathrm{~mL}, 4.6 \mathrm{mmol})$ in dry THF $(30 \mathrm{~mL}, 0.15 \mathrm{M})$ at $-78{ }^{\circ} \mathrm{C}$ and stirred for $2 \mathrm{~h}$ under argon. To the resulting LDA solution was added a solution of unsaturated cycloadduct $11(288.0 \mathrm{mg}, 1.2 \mathrm{mmol})$ in tetrahydrofuran $(4.6 \mathrm{~mL})$ and stirred for $20 \mathrm{~min}$. The pale yellow solution was treated with DMPU $(5.5 \mathrm{~mL})$ and stirred for $20 \mathrm{~min}$. followed by the addition of neat (3-bromo-1-propynyl)trimethyl silane $(1.1 \mathrm{~g}, 6.0 \mathrm{mmol})$. The resulting burgundy solution was gradually warmed to room temperature and stirred for $18 \mathrm{~h}$. The reaction mixture was cooled to $0{ }^{\circ} \mathrm{C}$, quenched with saturated $\mathrm{NH}_{4} \mathrm{Cl}$, and extracted with diethyl ether. The combined organic extracts were dried over $\mathrm{MgSO}_{4}$, filtered, and concentrated. Purification by silica gel chromatography (5\% Ether/hexane) afforded alkyne $\mathbf{2 0}(340 \mathrm{mg}, 82 \%)$ as a white solid: $\mathrm{mp} 80-82{ }^{\circ} \mathrm{C} ;{ }^{1} \mathrm{H}$ NMR $\left(500 \mathrm{MHz}, \mathrm{CDCl}_{3}\right) \delta 5.88(\mathrm{~s}, 1 \mathrm{H}), 5.18(\mathrm{~s}, 1 \mathrm{H}), 2.98(\mathrm{~d}, J=17.3 \mathrm{~Hz}, 1 \mathrm{H}), 2.86(\mathrm{~d}$, $J=17.3 \mathrm{~Hz}, 1 \mathrm{H}), 2.67-2.62(\mathrm{dd}, J=12.7,10.4 \mathrm{~Hz}, 1 \mathrm{H}), 2.28-2.25(\mathrm{~m}, 1 \mathrm{H}), 2.21-2.18(\mathrm{~m}$, 
$2 \mathrm{H}), 1.75-1.70(\mathrm{~m}, 4 \mathrm{H}), 1.63(\mathrm{~s}, 3 \mathrm{H}), 1.56(\mathrm{~s}, 3 \mathrm{H}), 1.50-1.43(\mathrm{~m}, 2 \mathrm{H}), 1.35-1.25(\mathrm{~m}, 1 \mathrm{H})$, 0.09 (s, 9H); ${ }^{13} \mathrm{C}$ NMR $\left(125 \mathrm{MHz}, \mathrm{CDCl}_{3}\right.$ ) $\delta 173.3,149.4,116.5,106.5,104.1,88.8,84.8$, 45.1, 43.9, 34.9, 34.3, 32.6, 32.1, 30.3, 29.1, 27.9, 27.7, -0.3; FTIR (thin film) 2926, 2854, 2177, 1738, 1518, 1444, 1388, 1377, 1320, 1282, 1250, 1178, 1106, $1045 \mathrm{~cm}^{-1}$. Exact mass calculated for $\mathrm{C}_{20} \mathrm{H}_{30} \mathrm{O}_{3} \mathrm{Si}+\mathrm{Na}$ (ES) 369.2953, found 369.1847.
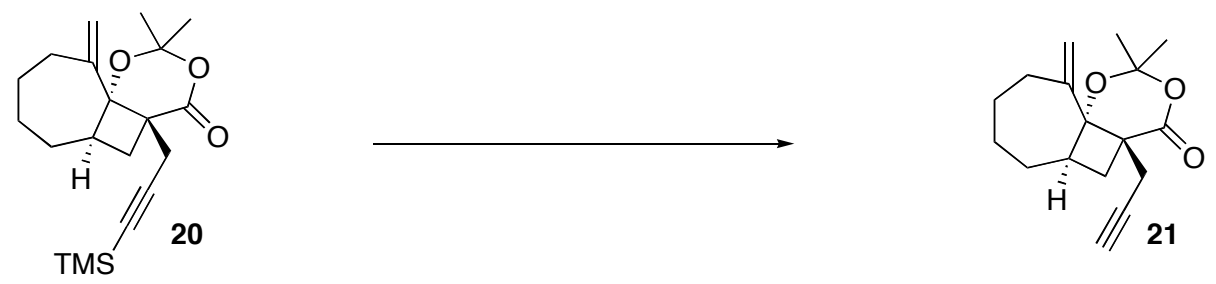

Enyne 21. A solution of alkyne $20(25.0 \mathrm{mg}, 0.07 \mathrm{mmol})$ in tetrahydrofuran $(2.0 \mathrm{~mL})$ was treated with tetrabutylammonium fluoride $(0.09 \mathrm{~mL}, 1.0 \mathrm{M}$ in THF) at room temperature and allowed to stir for $1.5 \mathrm{~h}$. Saturated $\mathrm{NH}_{4} \mathrm{Cl}$ was added and the reaction mixture was extracted with diethyl ether. The combined organic extracts were dried over $\mathrm{MgSO}_{4}$, filtered, and concentrated. Purification by silica gel chromatography (15\% Ether/Petroleum Ether) afforded enyne 21 (20.0 mg, $100 \%)$ as a white solid: mp 114-115 ${ }^{\circ} \mathrm{C},{ }^{1} \mathrm{H}$ NMR $\left(500 \mathrm{MHz}, \mathrm{CDCl}_{3}\right) \delta 5.69(\mathrm{~s}, 1 \mathrm{H}), 5.19(\mathrm{~s}, 1 \mathrm{H}), 2.97-2.93$ (dd, $J=17.2,2.7$ $\mathrm{Hz}, 1 \mathrm{H}), 2.87-2.80(\mathrm{dd}, J=17.2,2.5 \mathrm{~Hz}, 1 \mathrm{H}), 2.67-2.63(\mathrm{dd}, J=12.6,10.3 \mathrm{~Hz}, 1 \mathrm{H})$, 2.34-2.33 (m, $1 \mathrm{H}), 2.26-2.15(\mathrm{~m}, 2 \mathrm{H}), 2.14-2.13(\mathrm{dd}, J=2.6,2.6 \mathrm{~Hz}, 1 \mathrm{H}), 1.90-1.80(\mathrm{~m}$, $1 \mathrm{H}), 1.74-1.62(\mathrm{~m}, 2 \mathrm{H}), 1.60(\mathrm{~s}, 3 \mathrm{H}), 1.57(\mathrm{~s}, 3 \mathrm{H}), 1.54-1.44(\mathrm{~m}, 3 \mathrm{H}), 1.40-1.35(\mathrm{~m}, 1 \mathrm{H})$; ${ }^{13} \mathrm{C}$ NMR $\left(125 \mathrm{MHz}, \mathrm{CDCl}_{3}\right) \delta$ 173.2, 149.2, 116.8, 106.7, 84.9, 81.2, 72.0, 44.6, 44.2, 34.2 , 34.1, 32.7, 30.3, 29.7, 28.6, 27.9, 25.9; FTIR (thin film) 3274, 2994, 2923, 2852, 2359, 1733, 1684, 1636, 1456, 1443, 1388, 1378, 1321, 1284, 1260, 1234, 1178, 1106, $1038 \mathrm{~cm}^{-1}$. Exact mass calculated for $\mathrm{C}_{17} \mathrm{H}_{22} \mathrm{O}_{3}+\mathrm{H}(\mathrm{CI}) 275.1647$, found 275.1652.
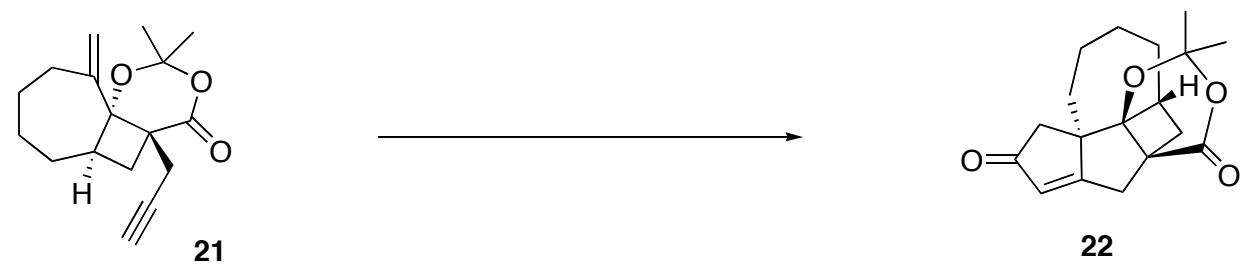

Cyclopentenone 22. A solution of enyne $21(30.0 \mathrm{mg}, 0.11 \mathrm{mmol})$ in toluene $(1.5 \mathrm{~mL}$, $0.73 \mathrm{M}$ ) was added to a flask containing 8.0 weight equivalence of flame-dried $4 \AA$ molecular sieves. Dicobaltoctacarbonyl $(45.0 \mathrm{mg}, 0.13 \mathrm{mmol})$ was added as a solid at room temperature and allowed to stir for $1.5 \mathrm{~h}$. The reaction mixture was cooled to -10 ${ }^{\circ} \mathrm{C}$ and a suspension of trimethylamine $\mathrm{N}$-oxide dihydrate $(75.0 \mathrm{mg}, 0.67 \mathrm{mmol})$ in 10.0 $\mathrm{mL}$ of toluene was added over an hour. The reaction was gradually warmed to room temperature and stirred under argon until the reaction was complete by TLC. The mixture was filtered through basic alumina and concentrated, and finally purified by silica gel chromatography (5\% ether/methylene chloride) to afford crystalline 
cyclopentanone $22(23.0 \mathrm{mg}, 69 \%)$ as a yellow solid: $\mathrm{mp} 148^{\circ}-152{ }^{\circ} \mathrm{C} ;{ }^{1} \mathrm{H}$ NMR (500 $\left.\mathrm{MHz}, \mathrm{CDCl}_{3}\right) \delta 5.79(\mathrm{~m}, 1 \mathrm{H}), 3.23-3.19(\mathrm{dd}, J=18.0,2.1 \mathrm{~Hz}, 1 \mathrm{H}), 2.99-2.93(\mathrm{~m}, 2 \mathrm{H})$, 2.92-2.88 (dd, $J=17.9,2.0 \mathrm{~Hz}, 1 \mathrm{H}), 2.51-2.47(\mathrm{dd}, J=11.5,11.5 \mathrm{~Hz}, 1 \mathrm{H}), 2.37(\mathrm{~d}, J=$ $17.7 \mathrm{~Hz}, 1 \mathrm{H}), 2.24-2.19(\mathrm{dd}, J=12.0,12.0 \mathrm{~Hz}, 1 \mathrm{H}), 2.04-1.99(\mathrm{~m}, 1 \mathrm{H}), 1.89-1.86(\mathrm{~m}$, $1 \mathrm{H}), 1.79-1.71(\mathrm{~m}, 1 \mathrm{H}), 1.67-1.63(\mathrm{~m}, 1 \mathrm{H}), 1.59(\mathrm{~s}, 3 \mathrm{H}), 1.56-1.45(\mathrm{~m}, 4 \mathrm{H}), 1.20(\mathrm{~s}, 3 \mathrm{H})$; ${ }^{13} \mathrm{C}$ NMR $\left(125 \mathrm{MHz}, \mathrm{CDCl}_{3}\right) \delta$ 209.6, 188.5, 174.0, 124.9, 106.9, 84.1, 62.1, 48.4, 45.7, 45.0, 36.4, 34.7, 31.2, 30.0, 29.7, 28.1, 27.8, 25.6; FTIR (thin film) 2993, 2927, 2855, 2359, 1733, 1701, 1628, 1471, 1439, 1408, 1389, 1377, 1316, 1296, 1255, 1203, 1183, $1152,1140,1126,1038 \mathrm{~cm}^{-1}$. Exact mass calculated for $\mathrm{C}_{18} \mathrm{H}_{22} \mathrm{O}_{3}+\mathrm{H}(\mathrm{CI}) 303.1596$, found 303.1601 .
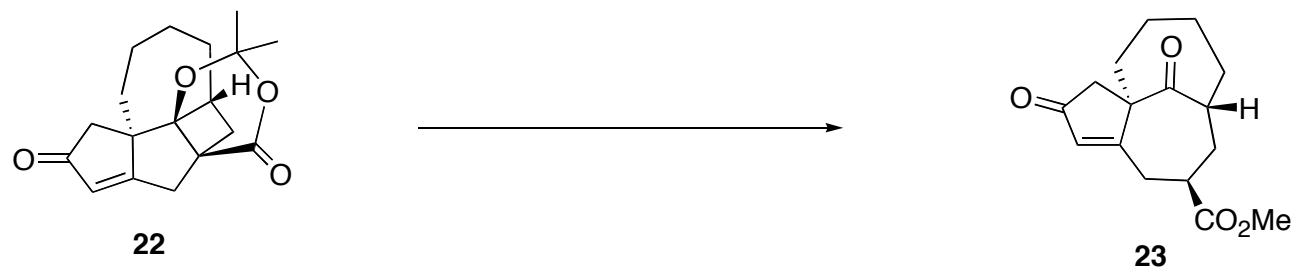

Isoingenane 23. A solution of cyclopentanone $22(4.0 \mathrm{mg}, 0.01 \mathrm{mmol})$ in anhydrous methanol $(0.25 \mathrm{~mL}, 0.05 \mathrm{M})$ was cooled to $0{ }^{\circ} \mathrm{C}$. Potassium carbonate $(1.6 \mathrm{mg}, 0.01$ $\mathrm{mmol})$ was added as a solid followed by anhydrous methanol $(0.2 \mathrm{~mL}, 0.05 \mathrm{M})$. The reaction was stirred at room temperature for $8 \mathrm{~h}$. The reaction was quenched with saturated $\mathrm{NH}_{4} \mathrm{Cl}$ and extracted with ethyl acetate. The combined organic layers were dried with $\mathrm{MgSO}_{4}$, filtered, and concentrated which afforded isoingenane $\mathbf{2 3}(2.0 \mathrm{mg}$, $55 \%)$ as crystalline solid: mp $116^{\circ}-120{ }^{\circ} \mathrm{C} ;{ }^{1} \mathrm{H}$ NMR $\left(500 \mathrm{MHz}, \mathrm{CDCl}_{3}\right) \delta 5.90(\mathrm{~s}, 1 \mathrm{H})$, $3.61(\mathrm{~s}, 3 \mathrm{H}), 3.60(\mathrm{~m}, 1 \mathrm{H}), 3.29(\mathrm{dd}, J=15.6,3.2 \mathrm{~Hz}, 1 \mathrm{H}), 3.20(\mathrm{~m}, 1 \mathrm{H}), 3.10(\mathrm{~m}, 1 \mathrm{H})$, $2.85(\mathrm{dd}, J=15.6,4.6 \mathrm{~Hz}, 1 \mathrm{H}), 2.43(\mathrm{~m}, 2 \mathrm{H}), 2.26(\mathrm{~m}, 1 \mathrm{H}), 1.96(\mathrm{~m}, 2 \mathrm{H}), 1.81(\mathrm{~m}, 5 \mathrm{H})$, $1.14(\mathrm{~m}, 1 \mathrm{H}) ;{ }^{13} \mathrm{C}$ NMR $\left(125 \mathrm{MHz}, \mathrm{CDCl}_{3}\right) \delta 209.0,207.1,176.3,172.6,132.2,65.7$, 51.9, 50.7, 46.9, 40.5, 35.7, 32.7, 32.0, 27.4, 25.7, 25.6; FTIR (thin film) 2926, 2855, $1732,1695,1609,1441,1196,1031 \mathrm{~cm}^{-1}$. Exact mass calculated for $\mathrm{C}_{16} \mathrm{H}_{20} \mathrm{O}_{4}+\mathrm{Na}(\mathrm{ES})$ 299.1259 , found 299.1269 . 


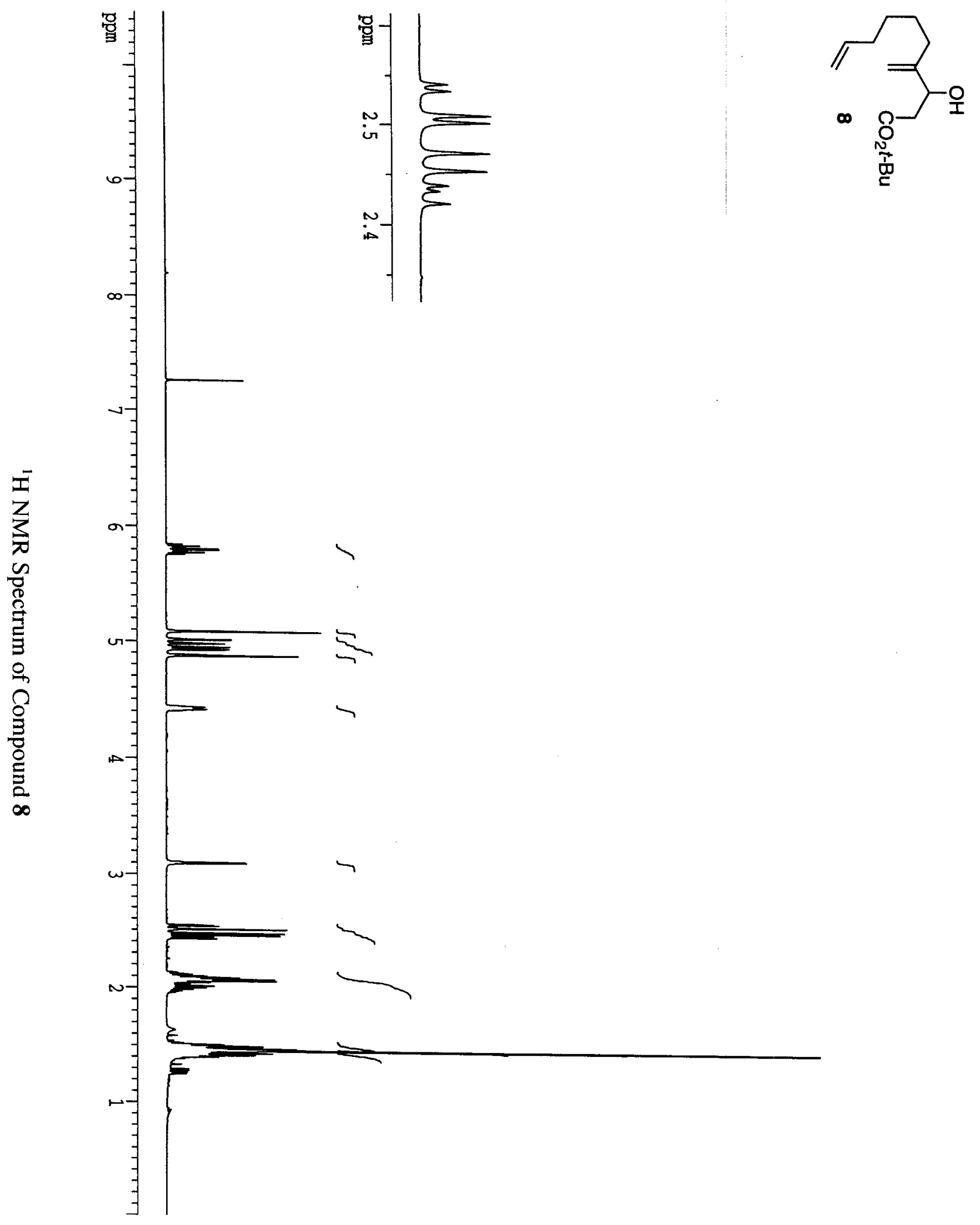




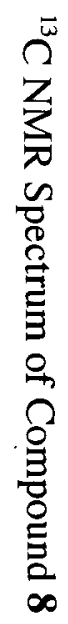
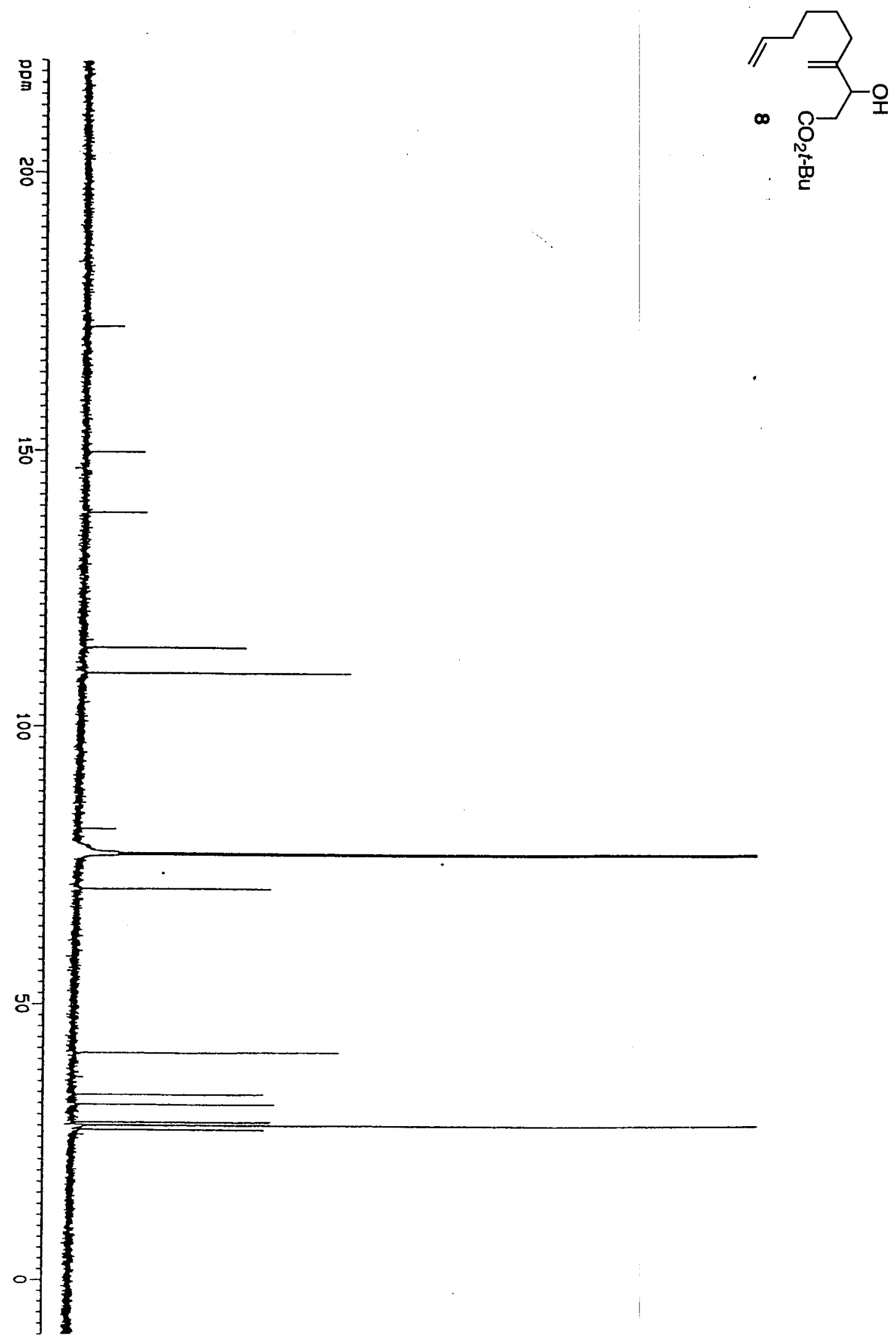

S-12 


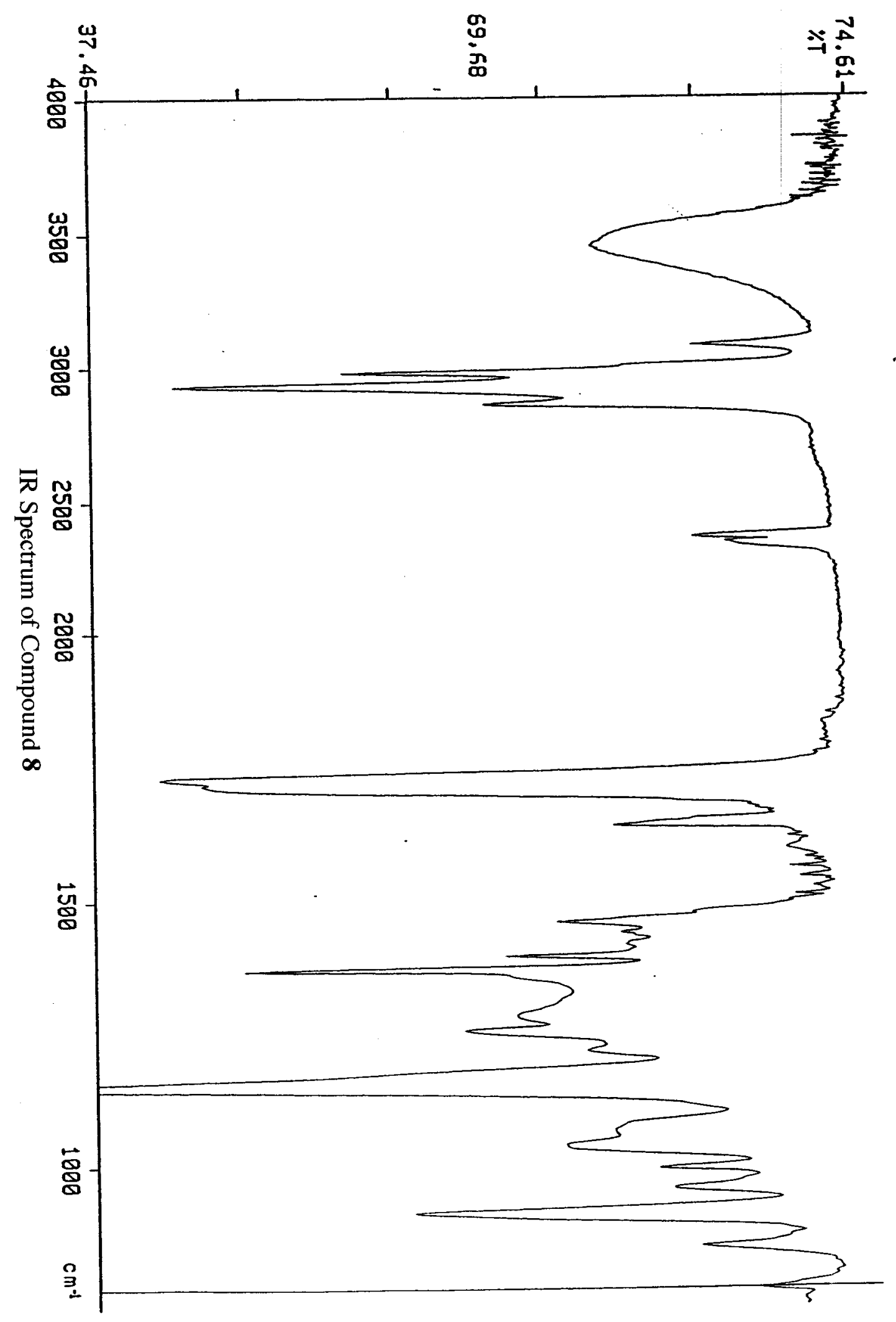

S-13 


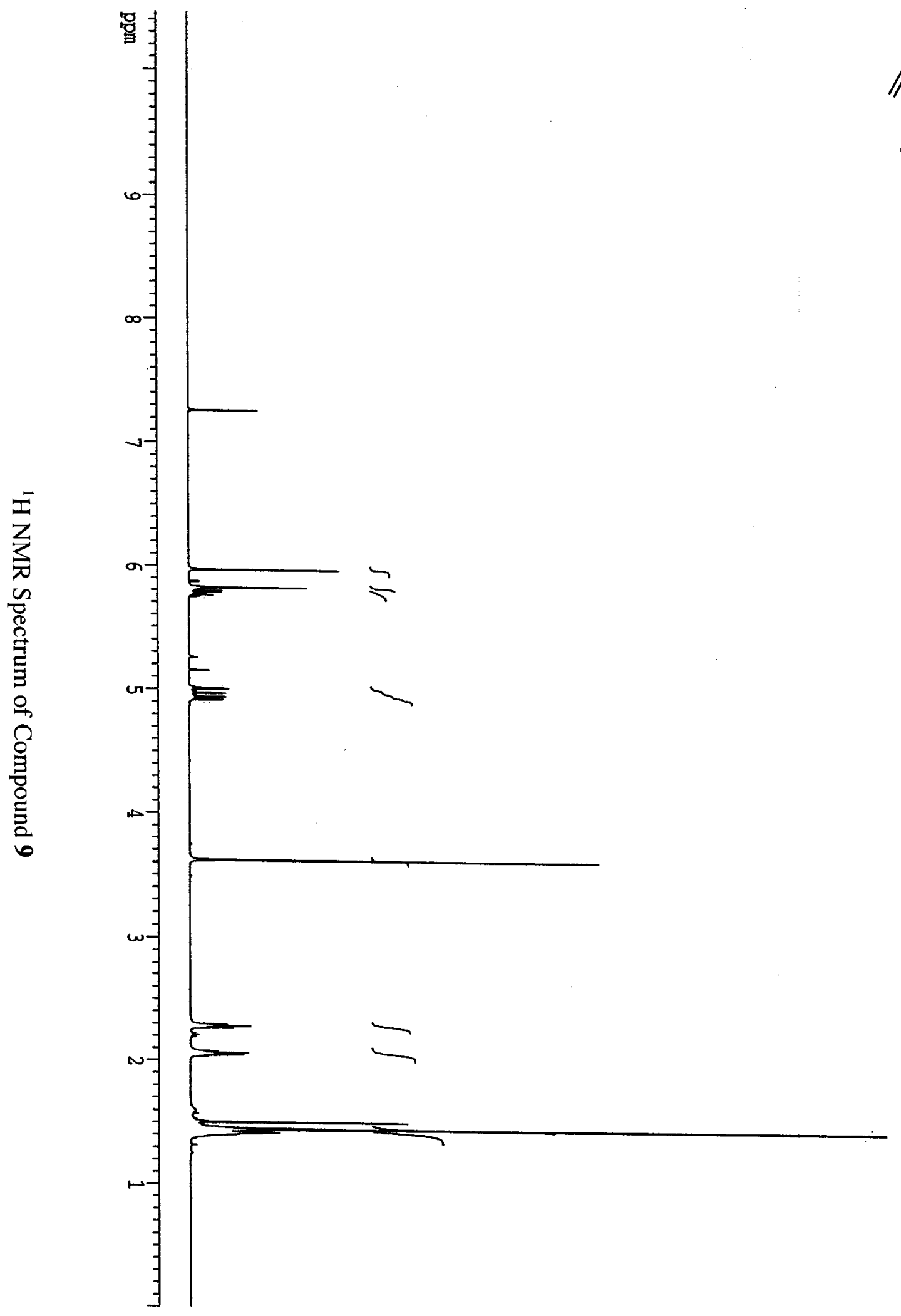

S-14 


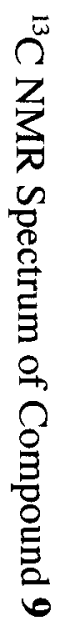

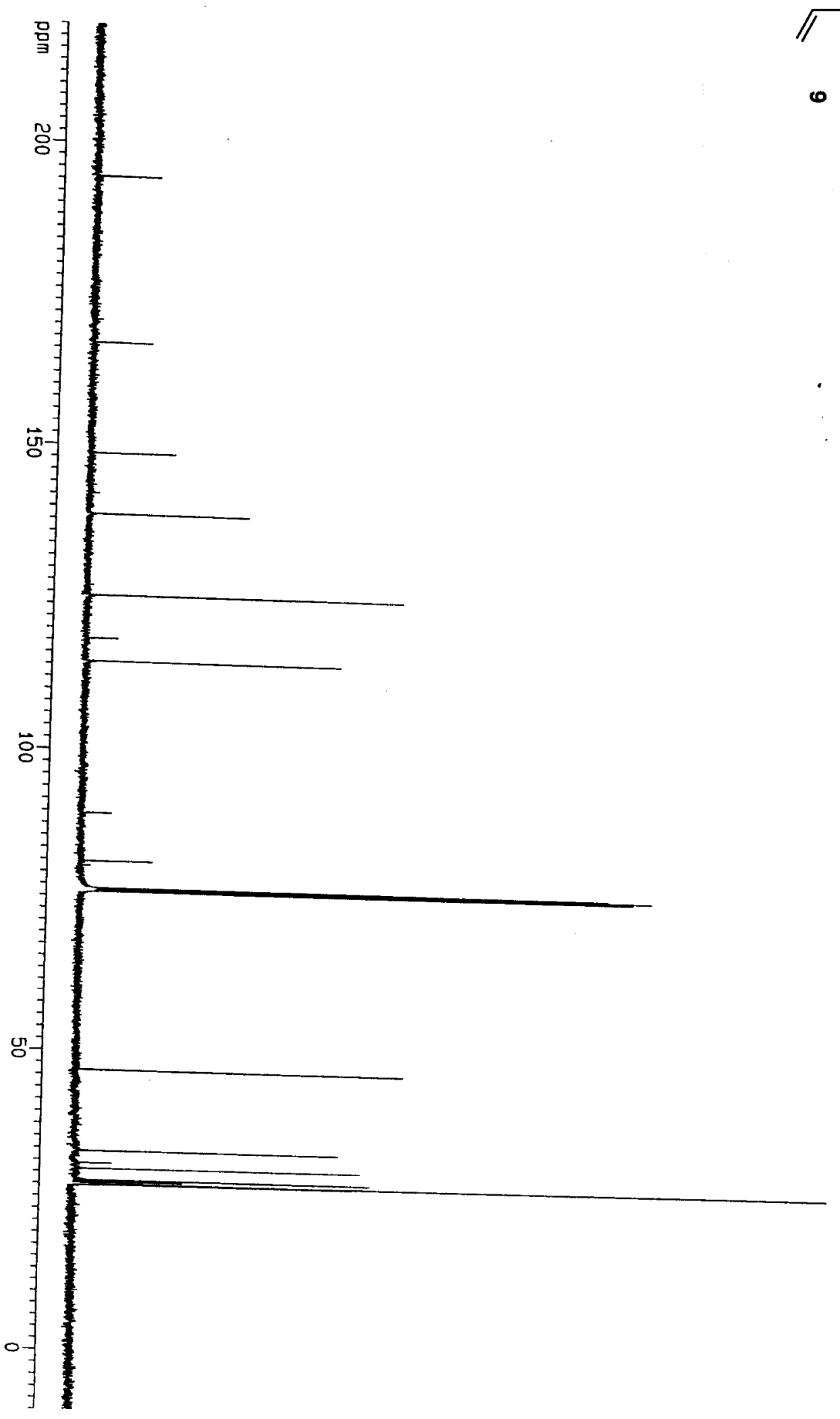

S-15 


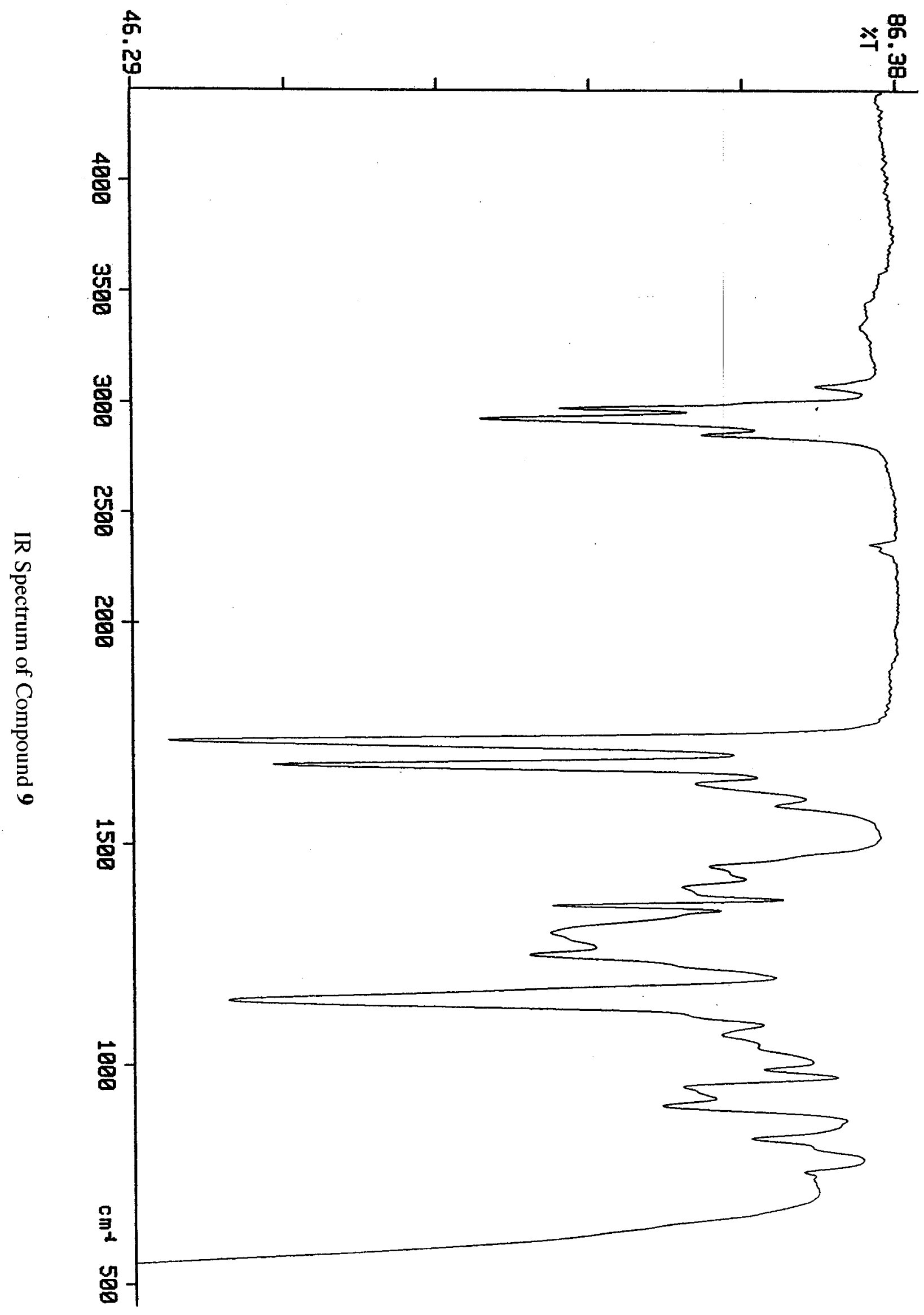

S-16 


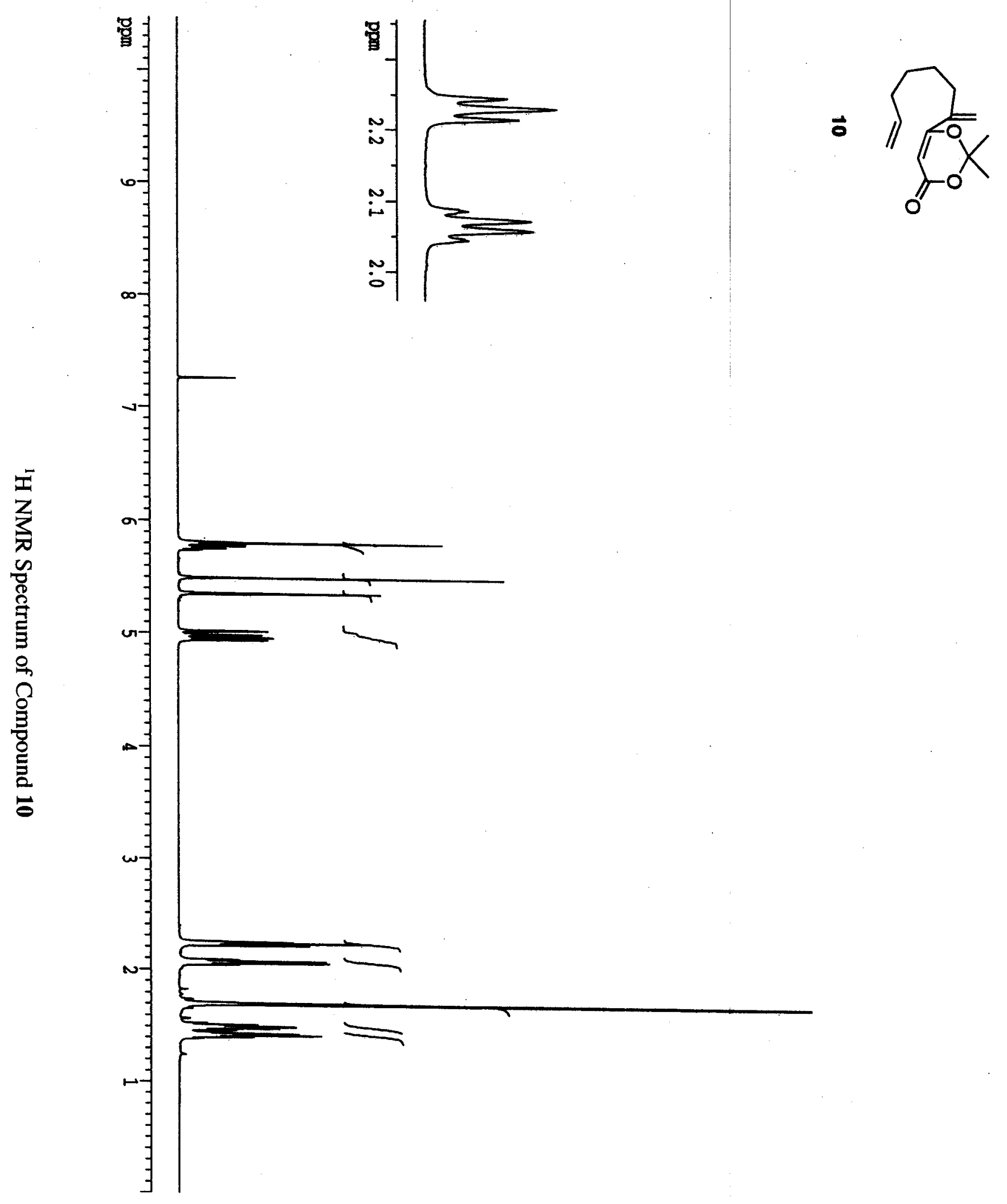




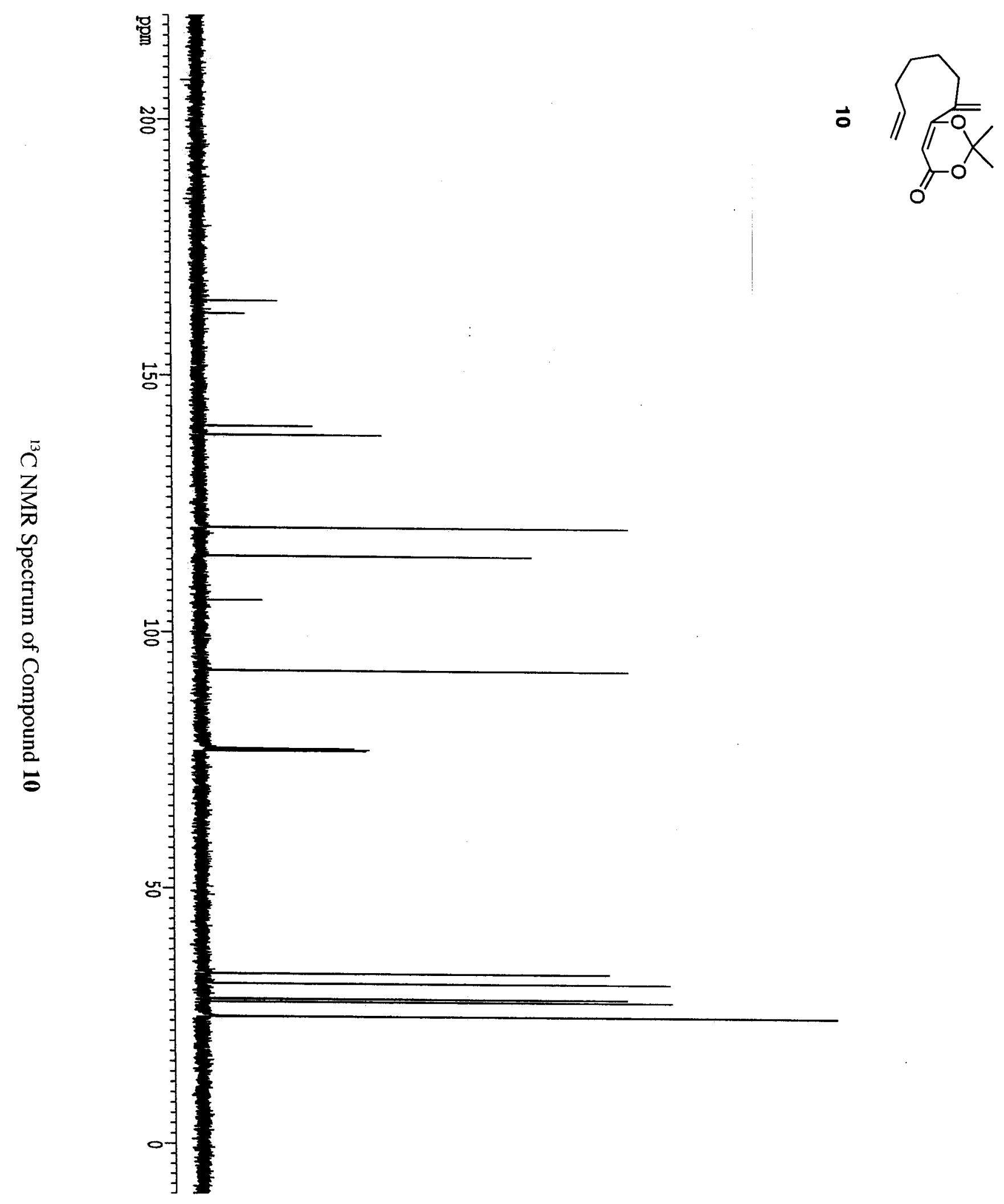




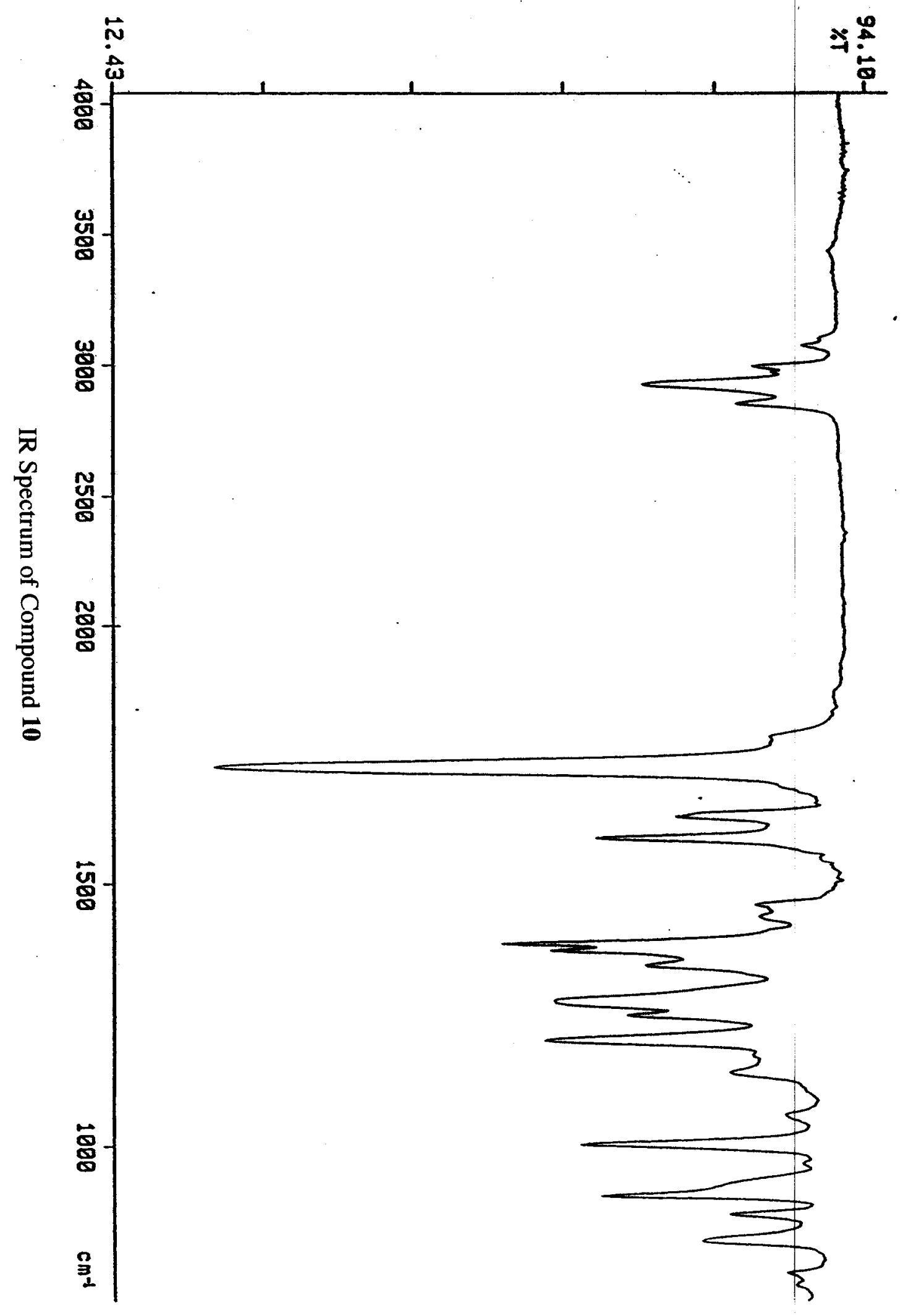

S-19 


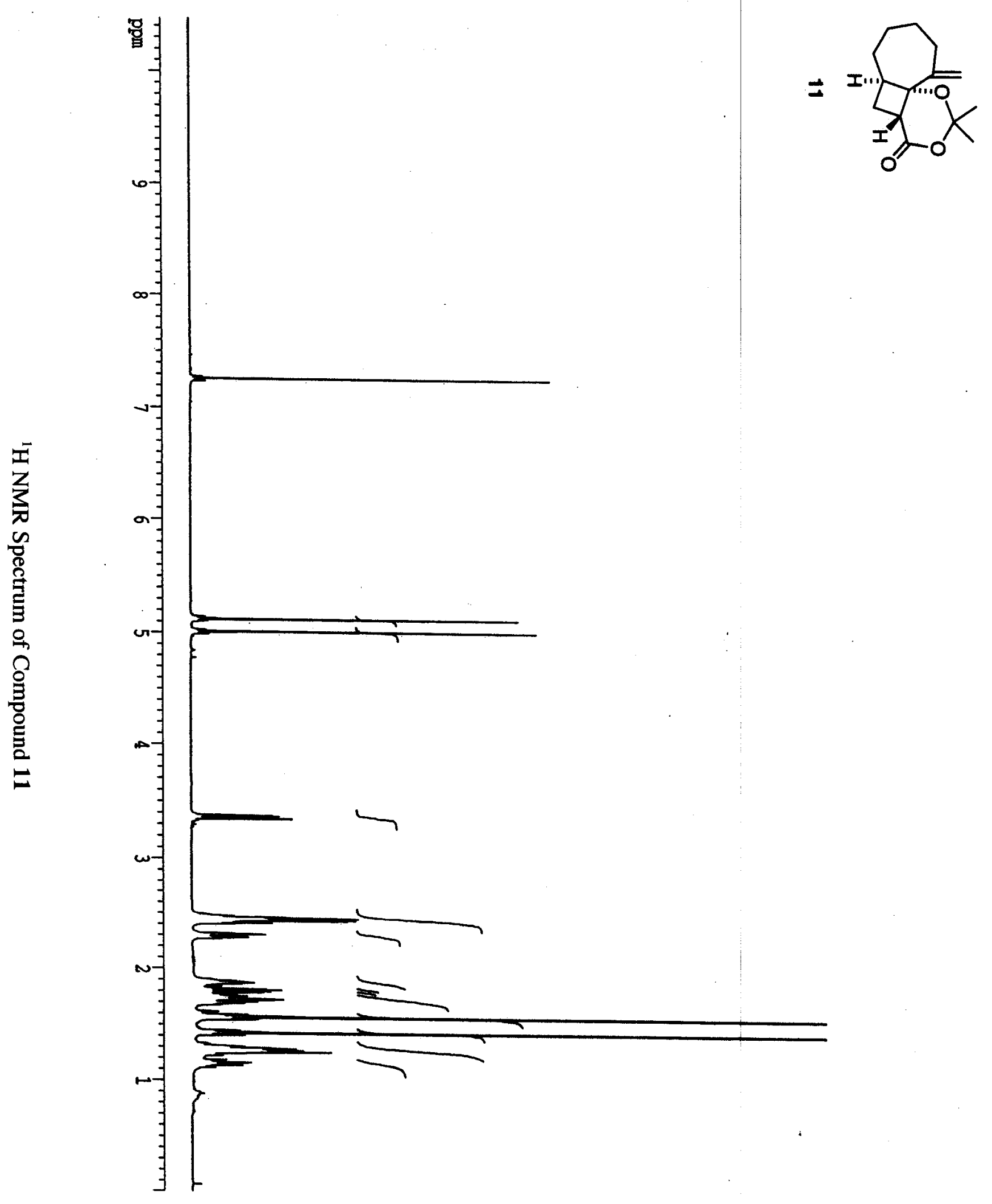




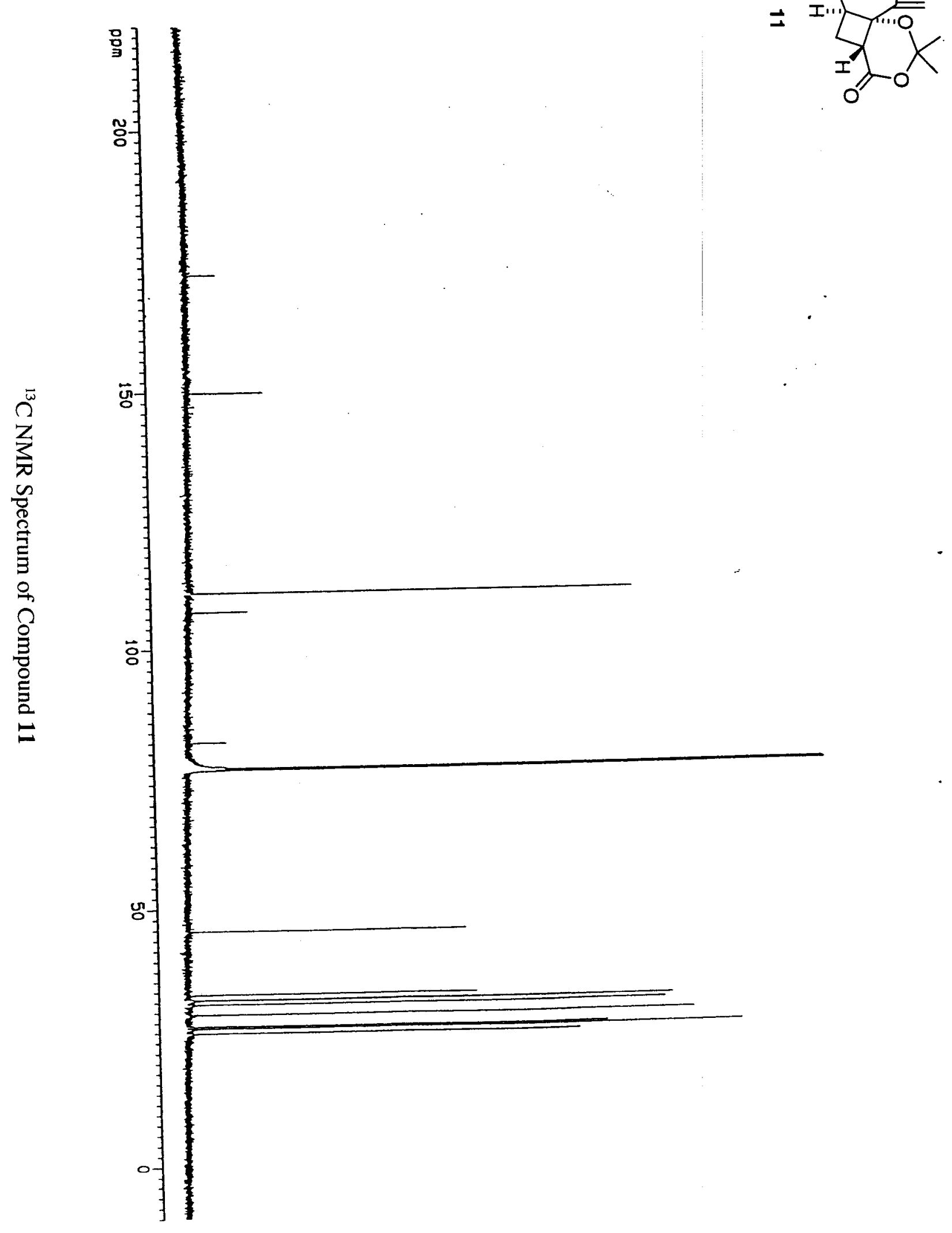

S-21 


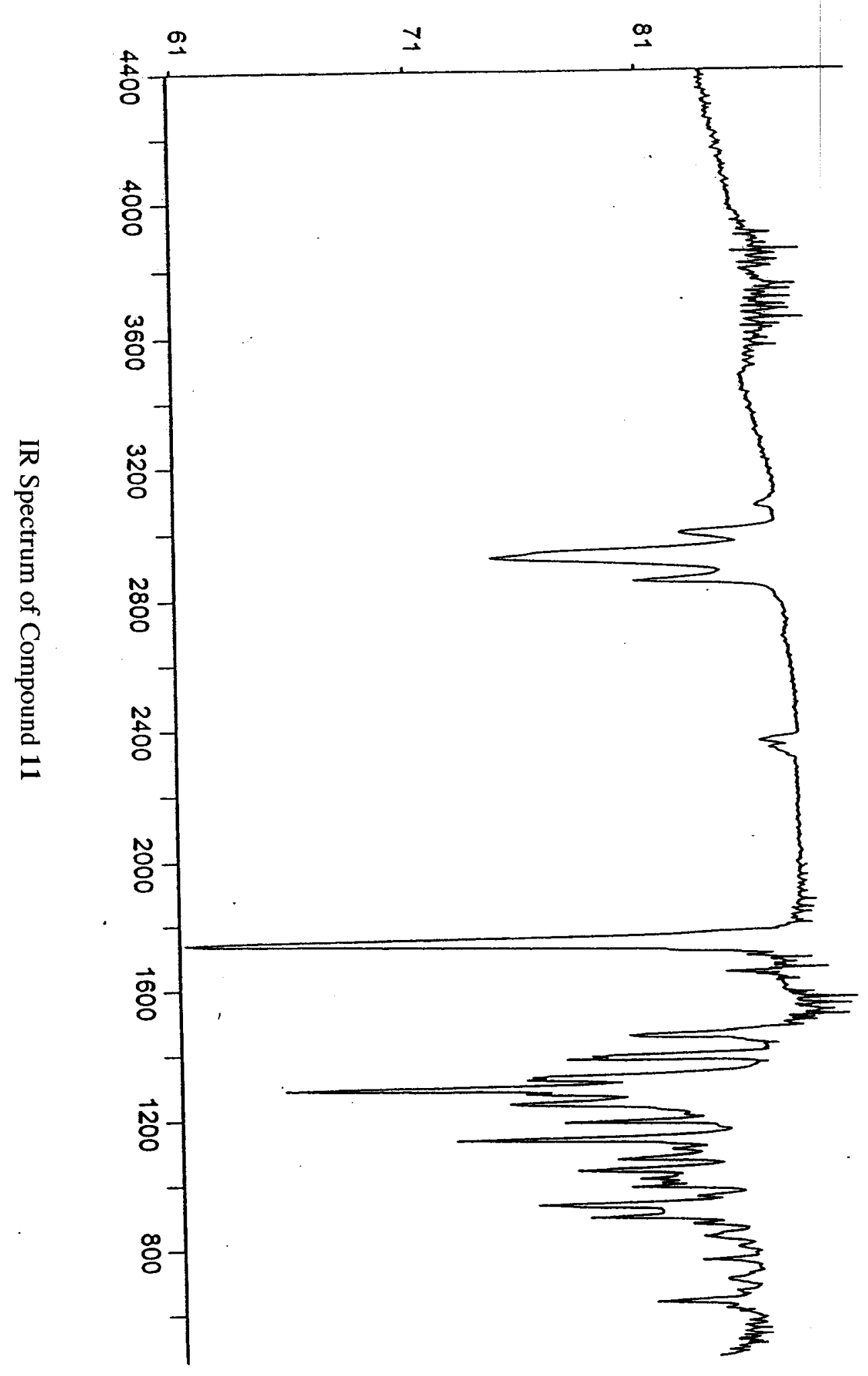

S-22 


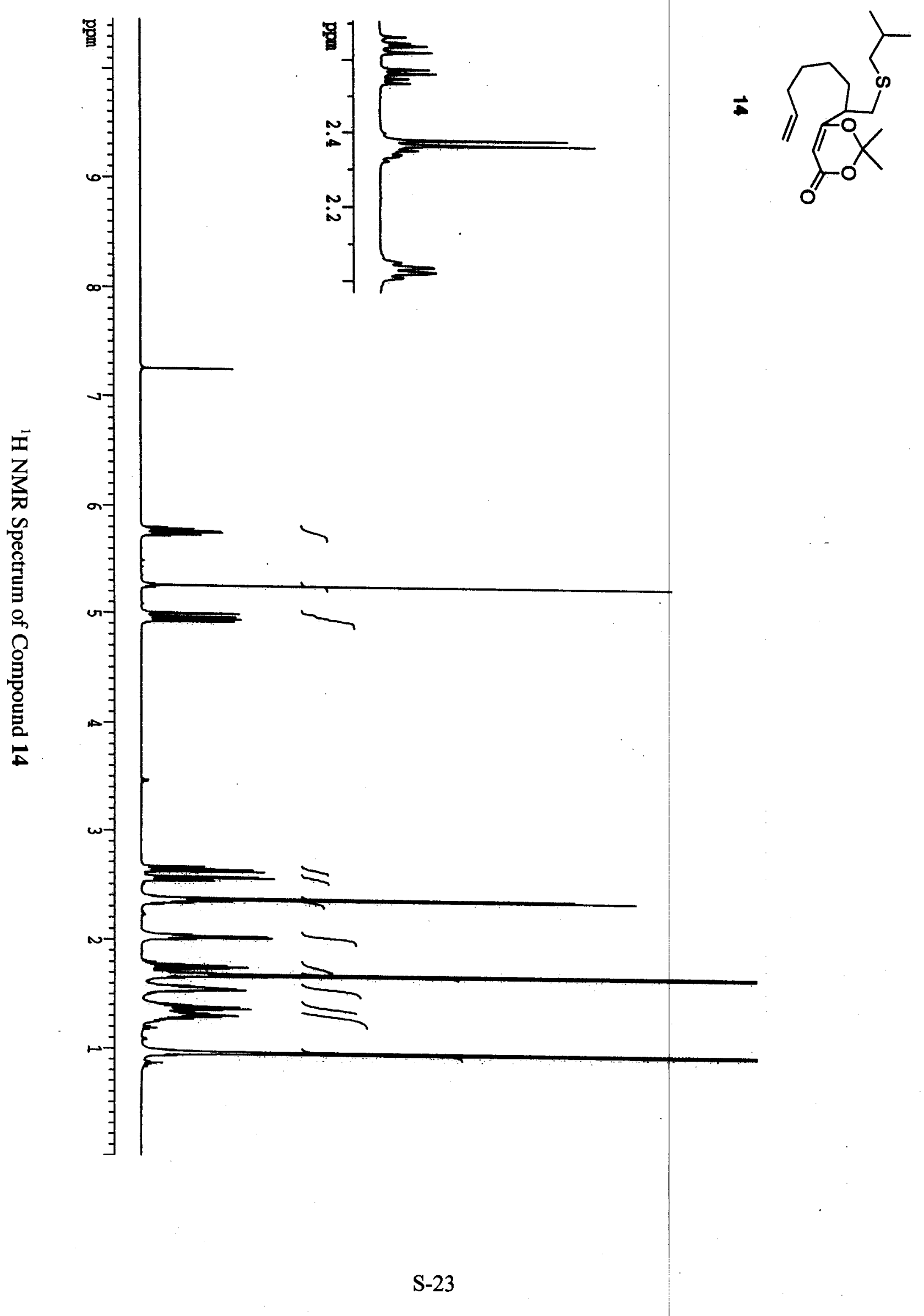




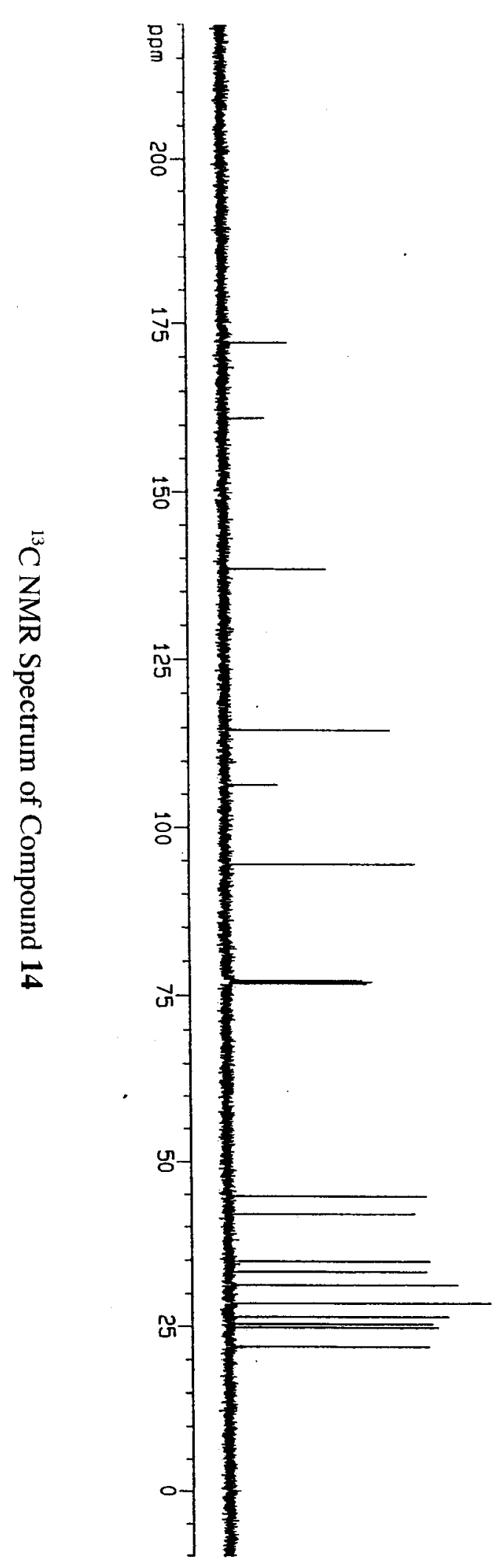




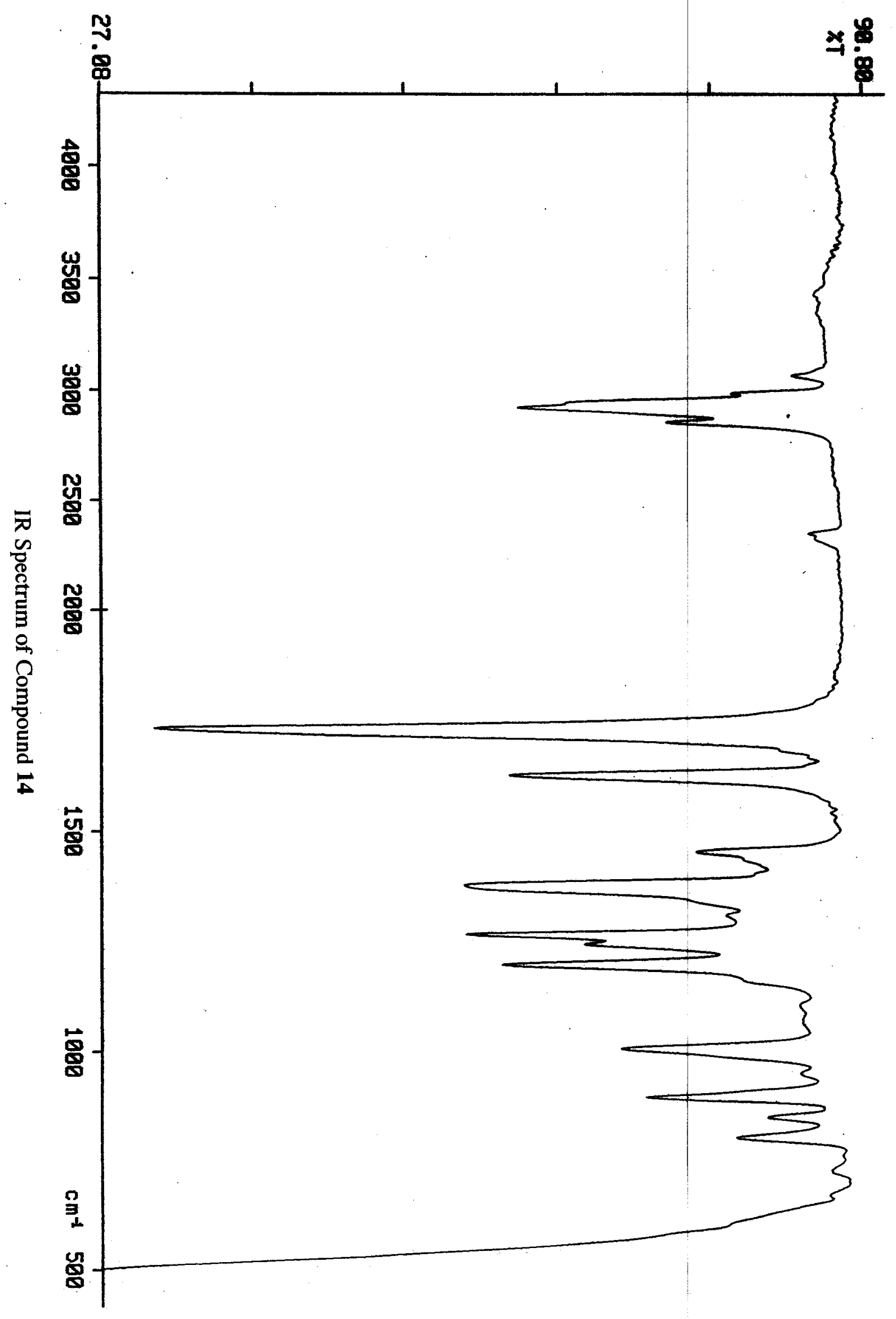

S-25 


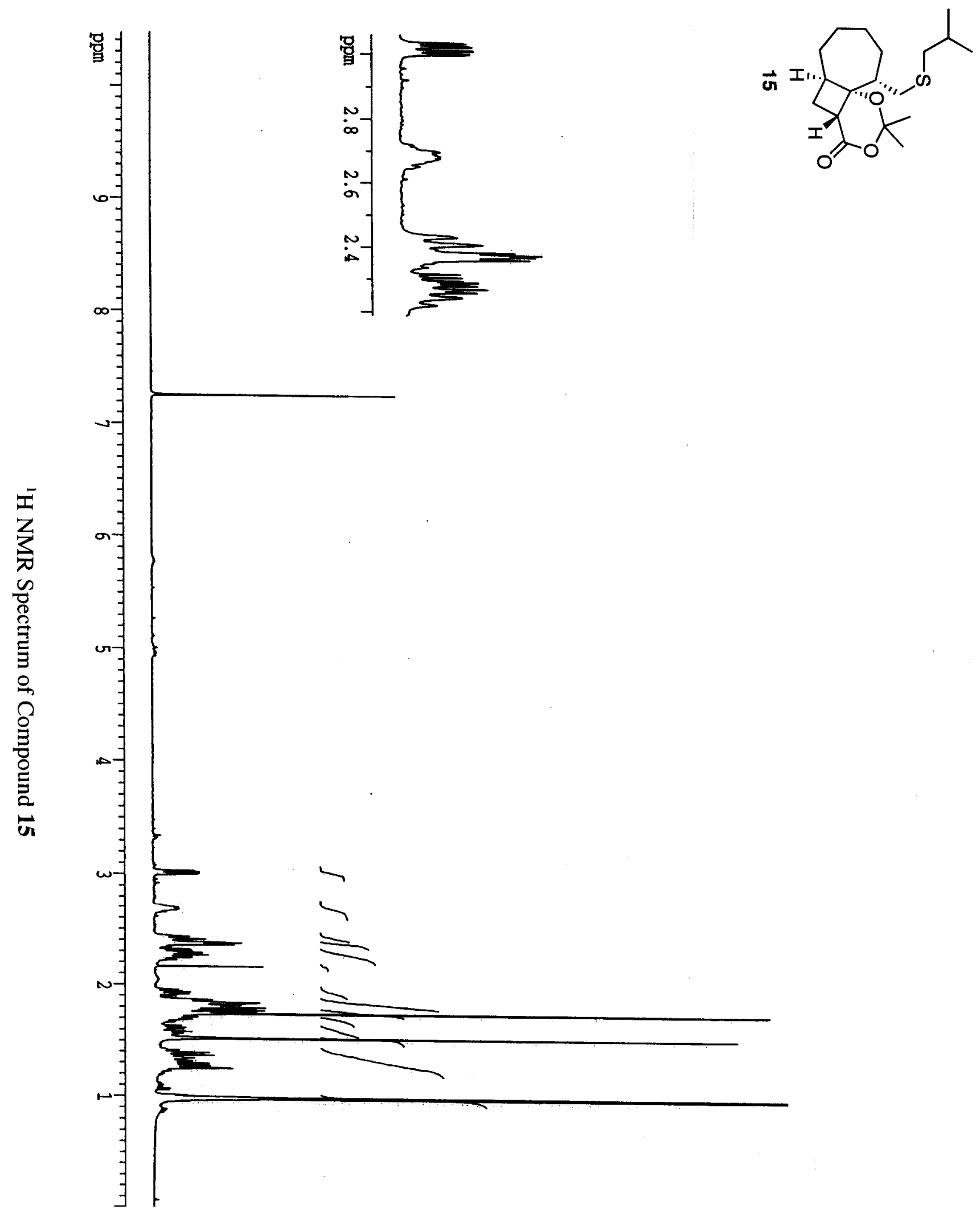



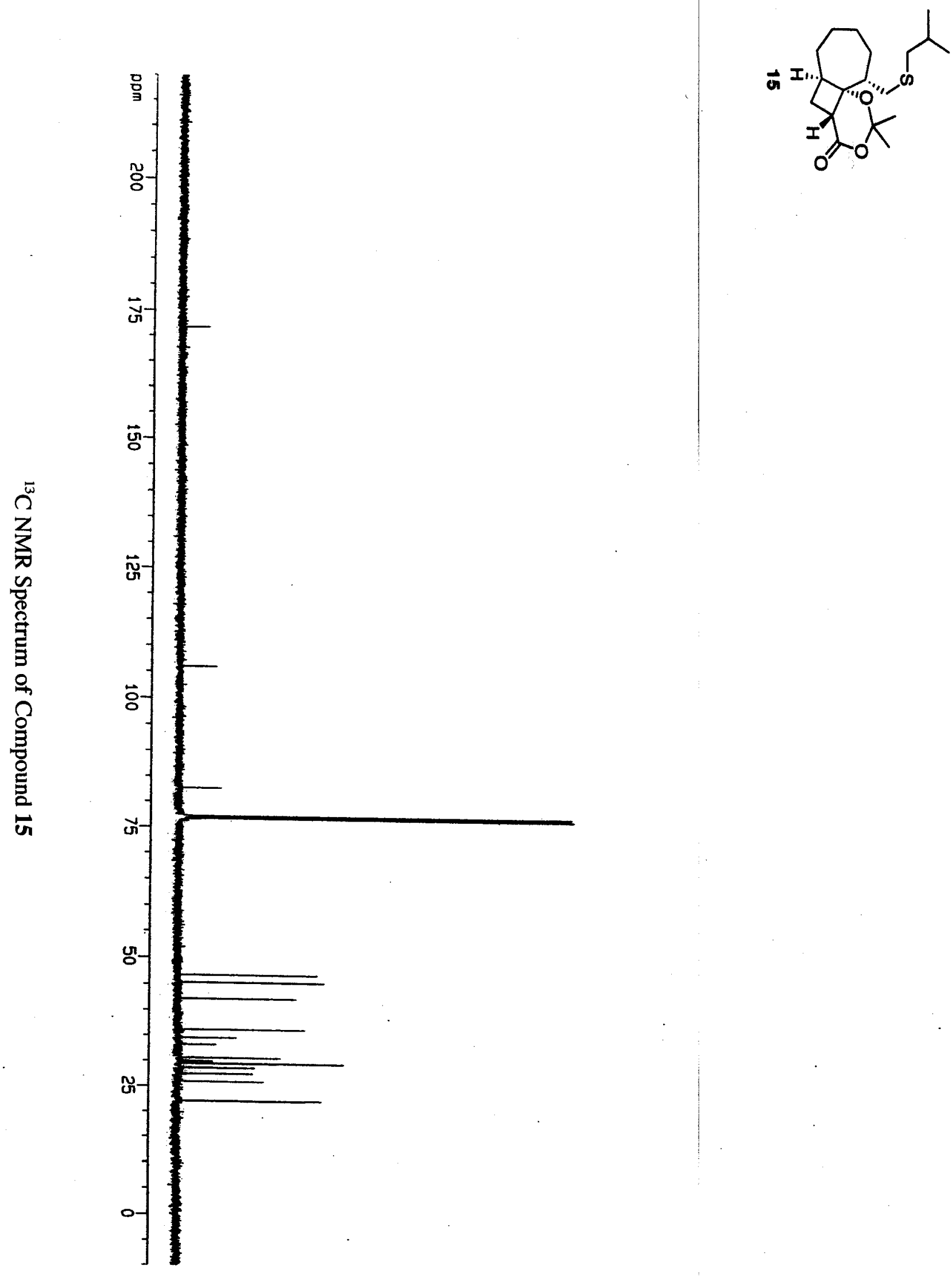


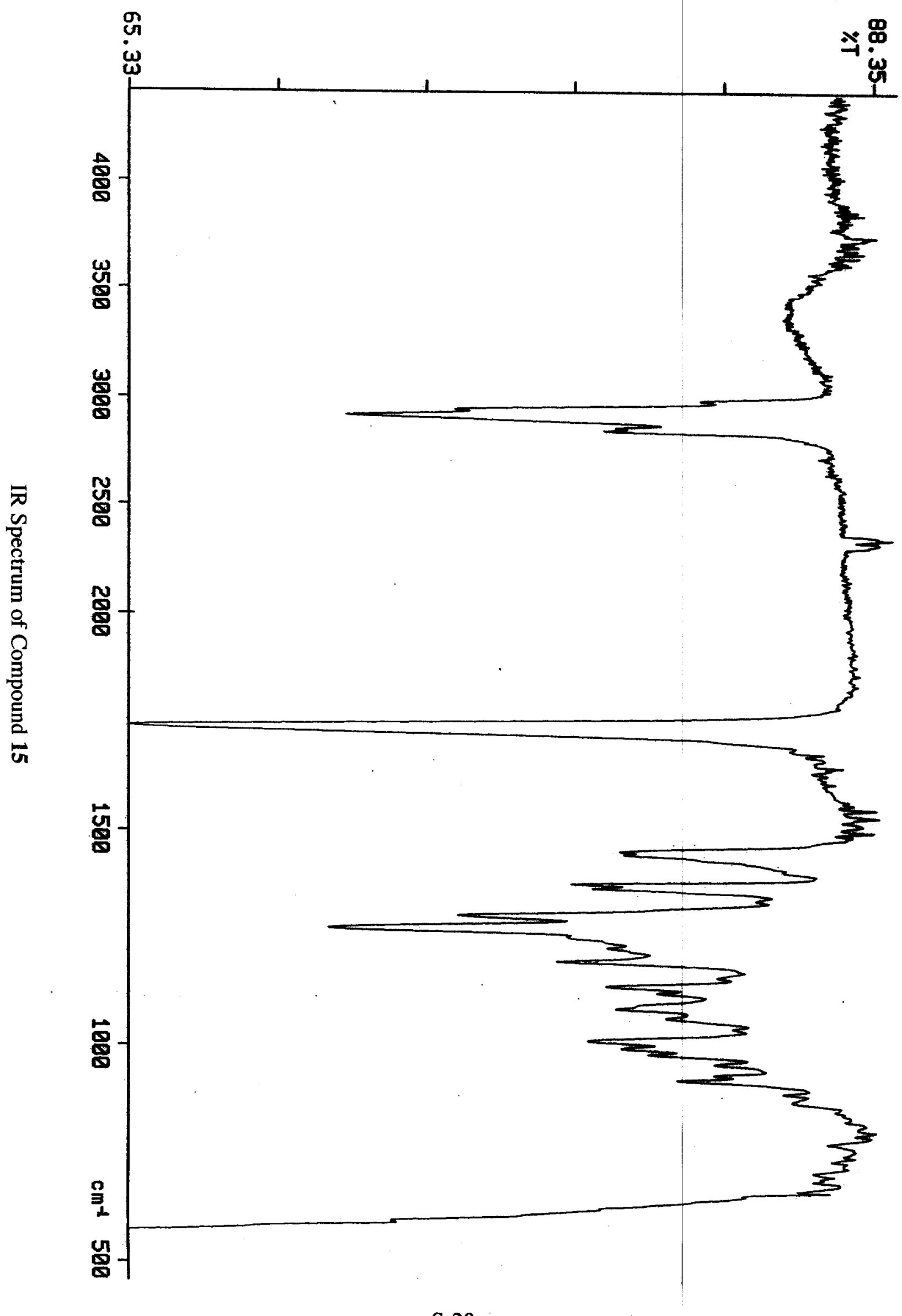




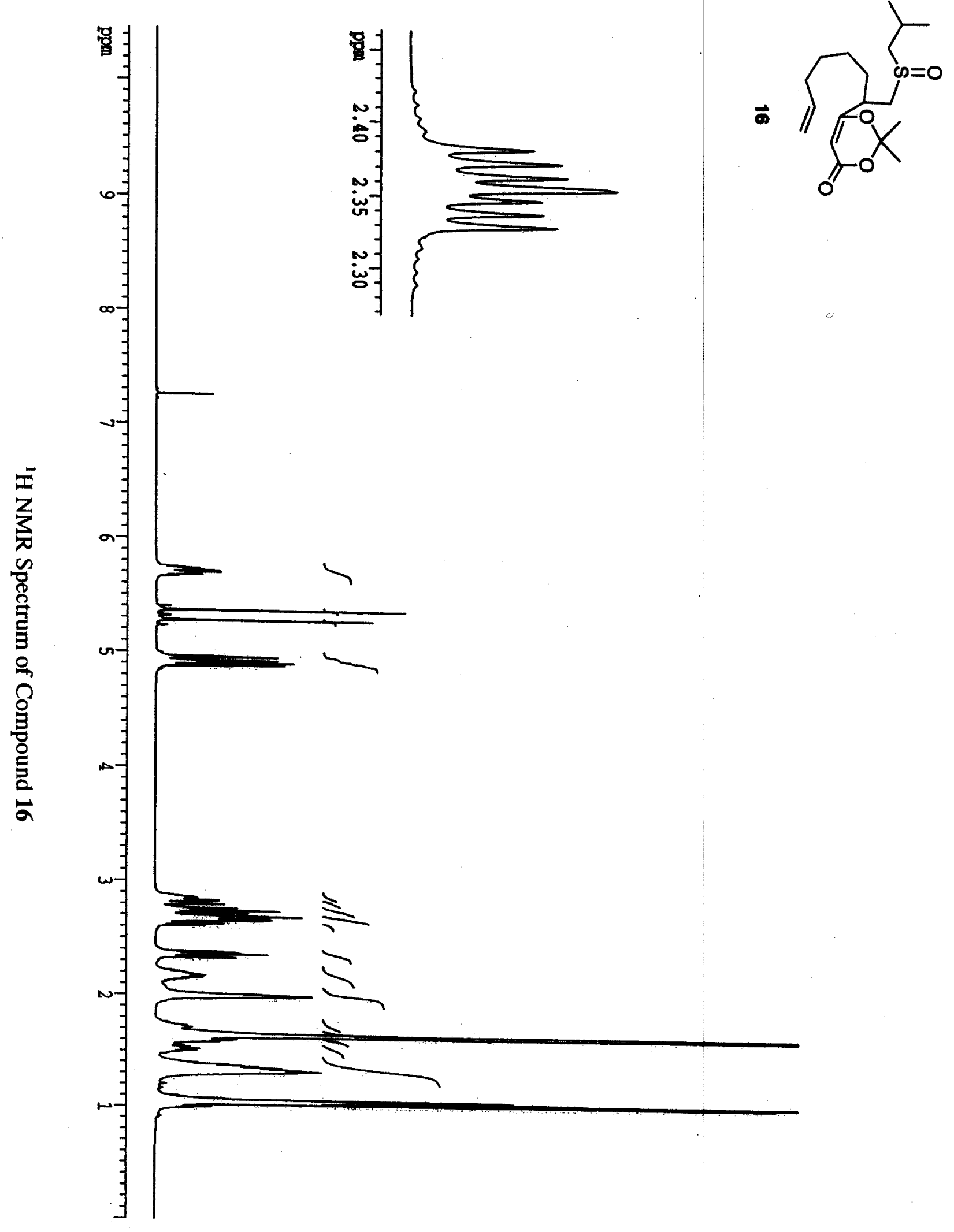



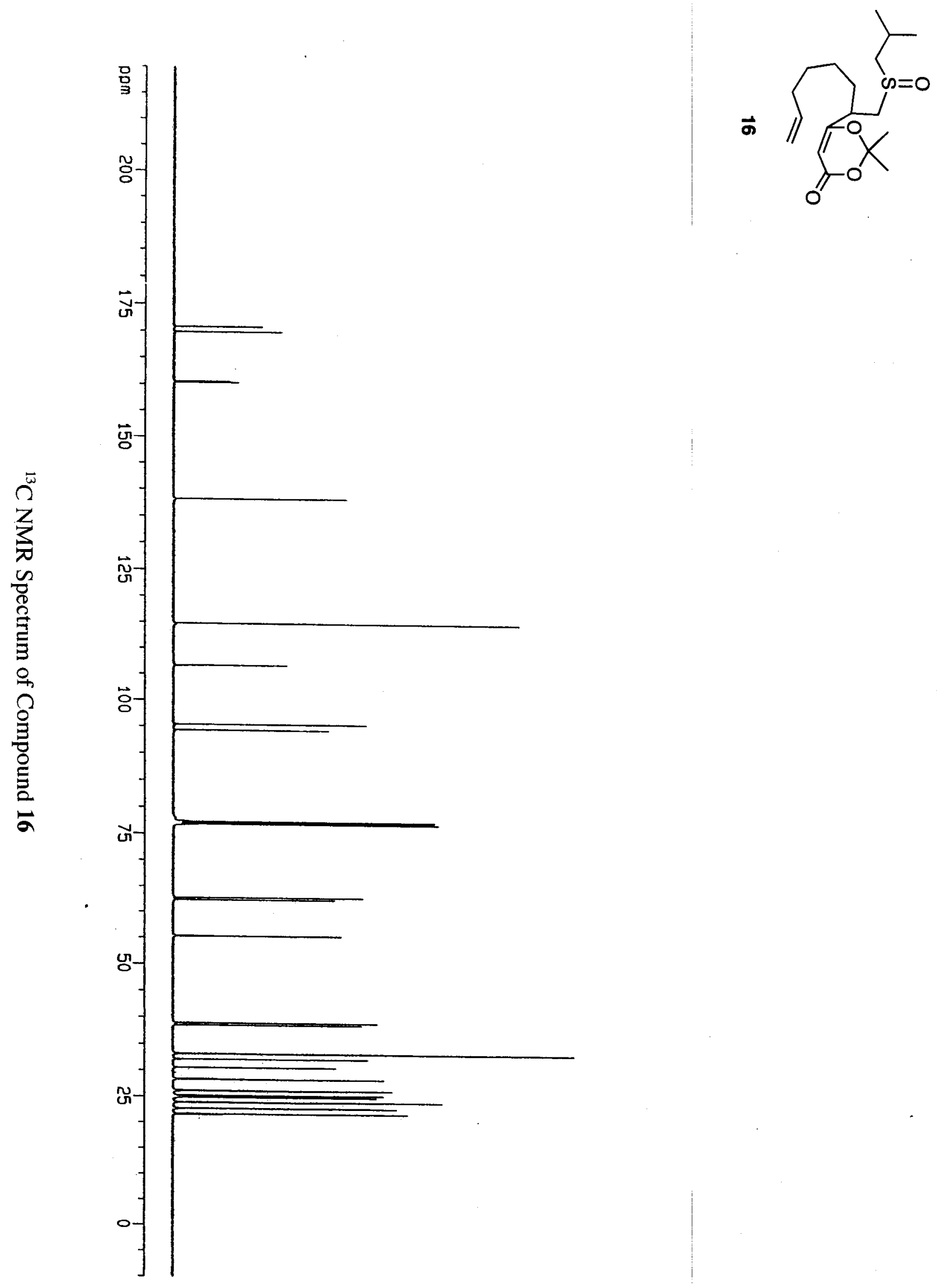


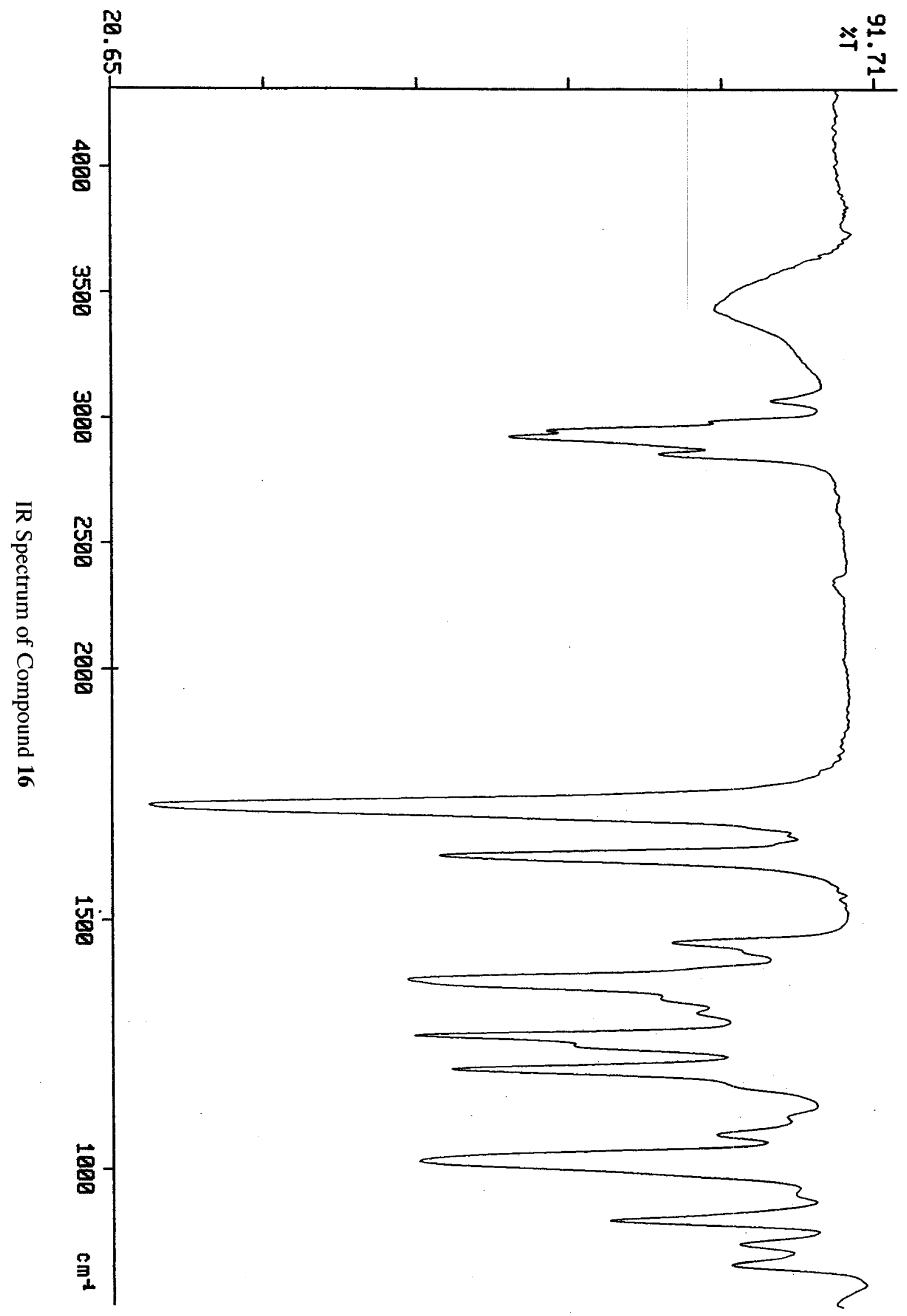

S-31 


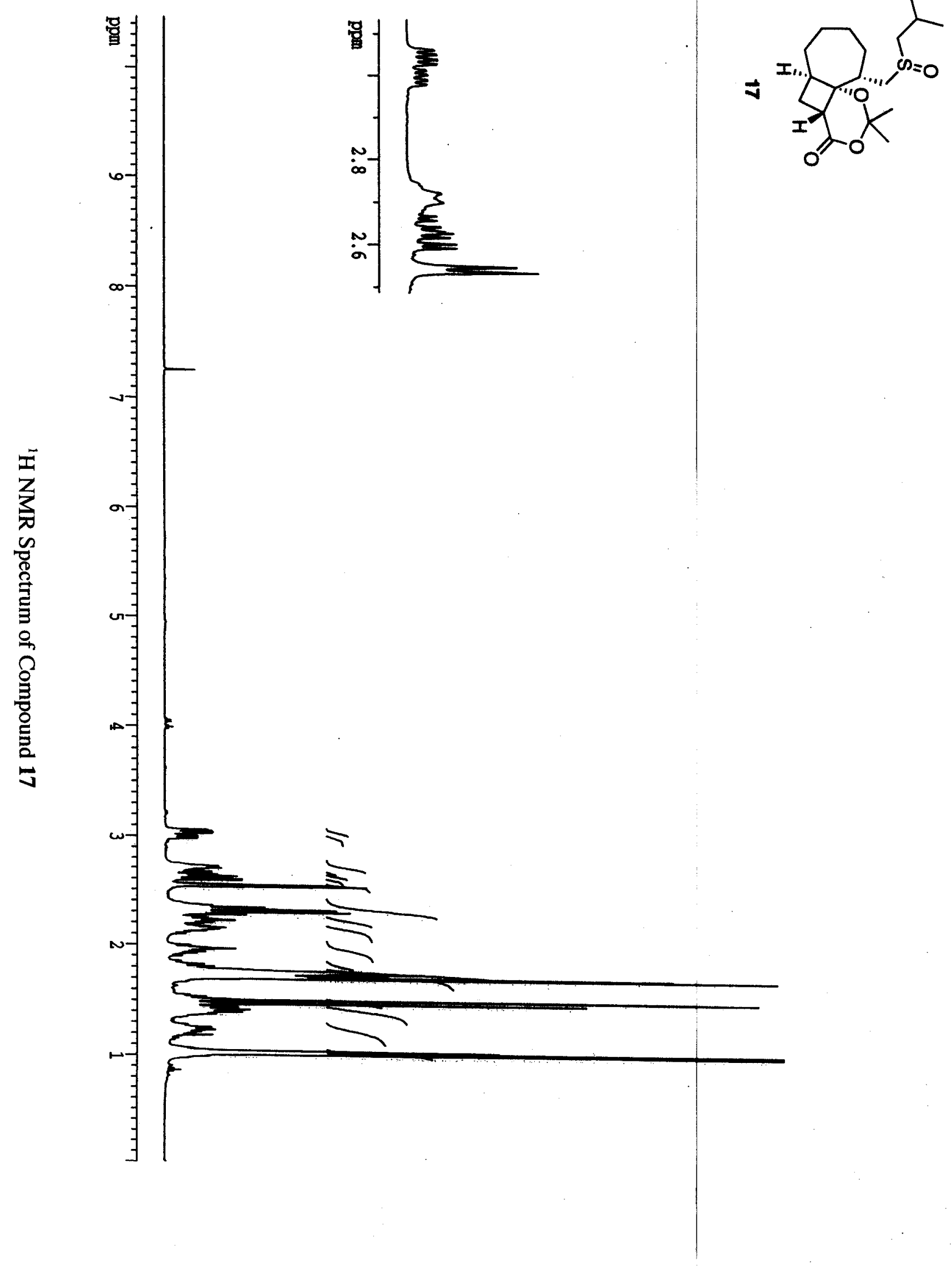




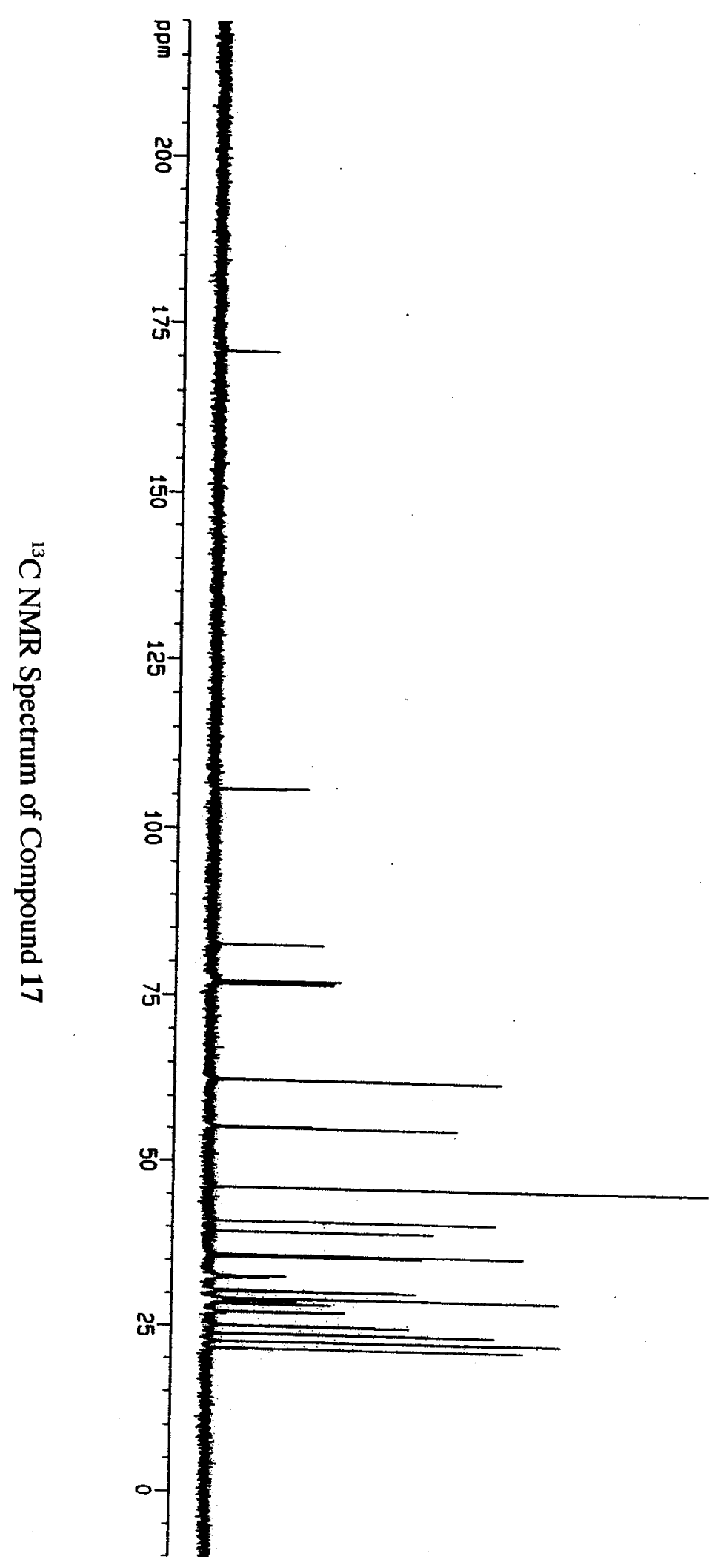




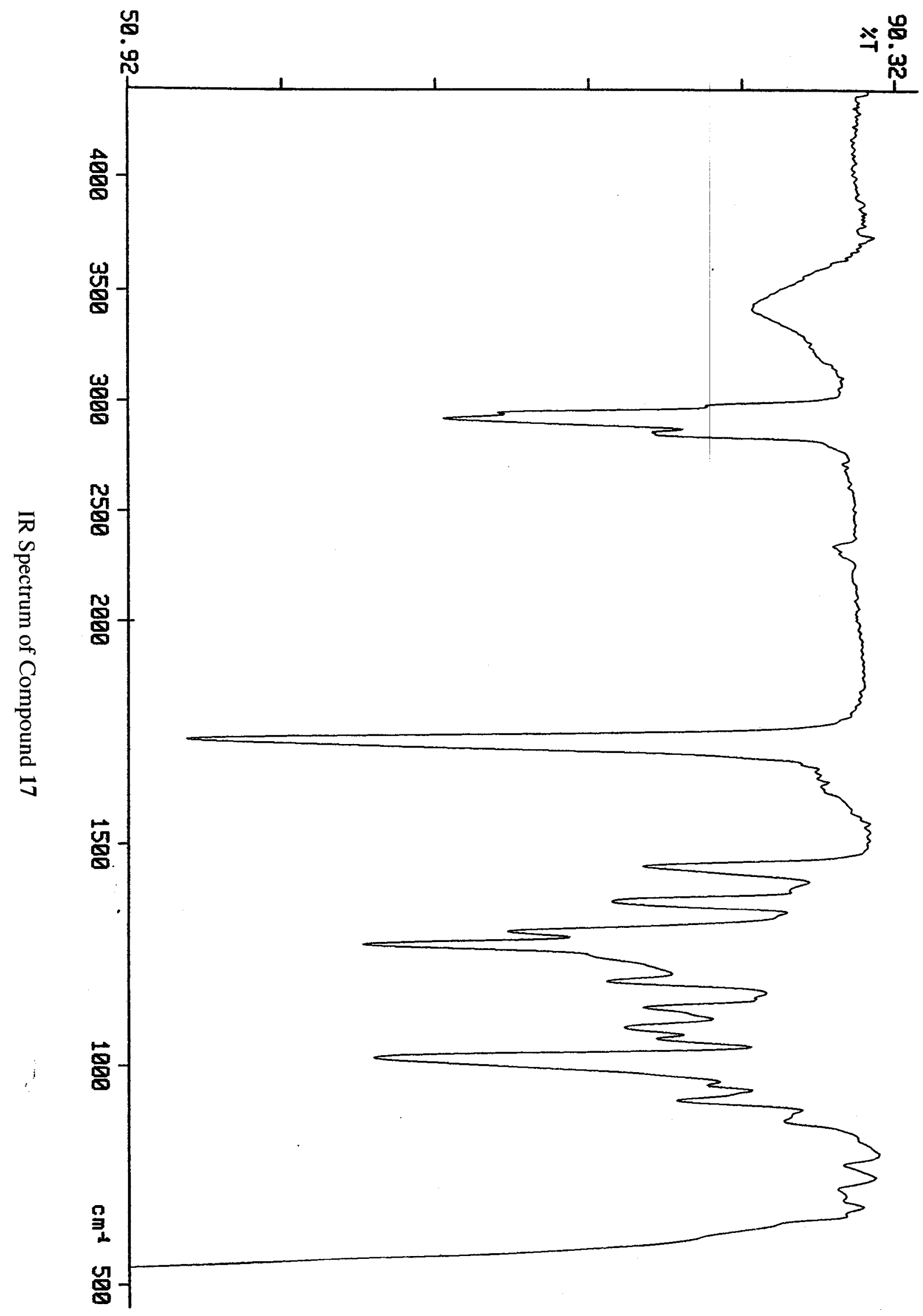

S-34 


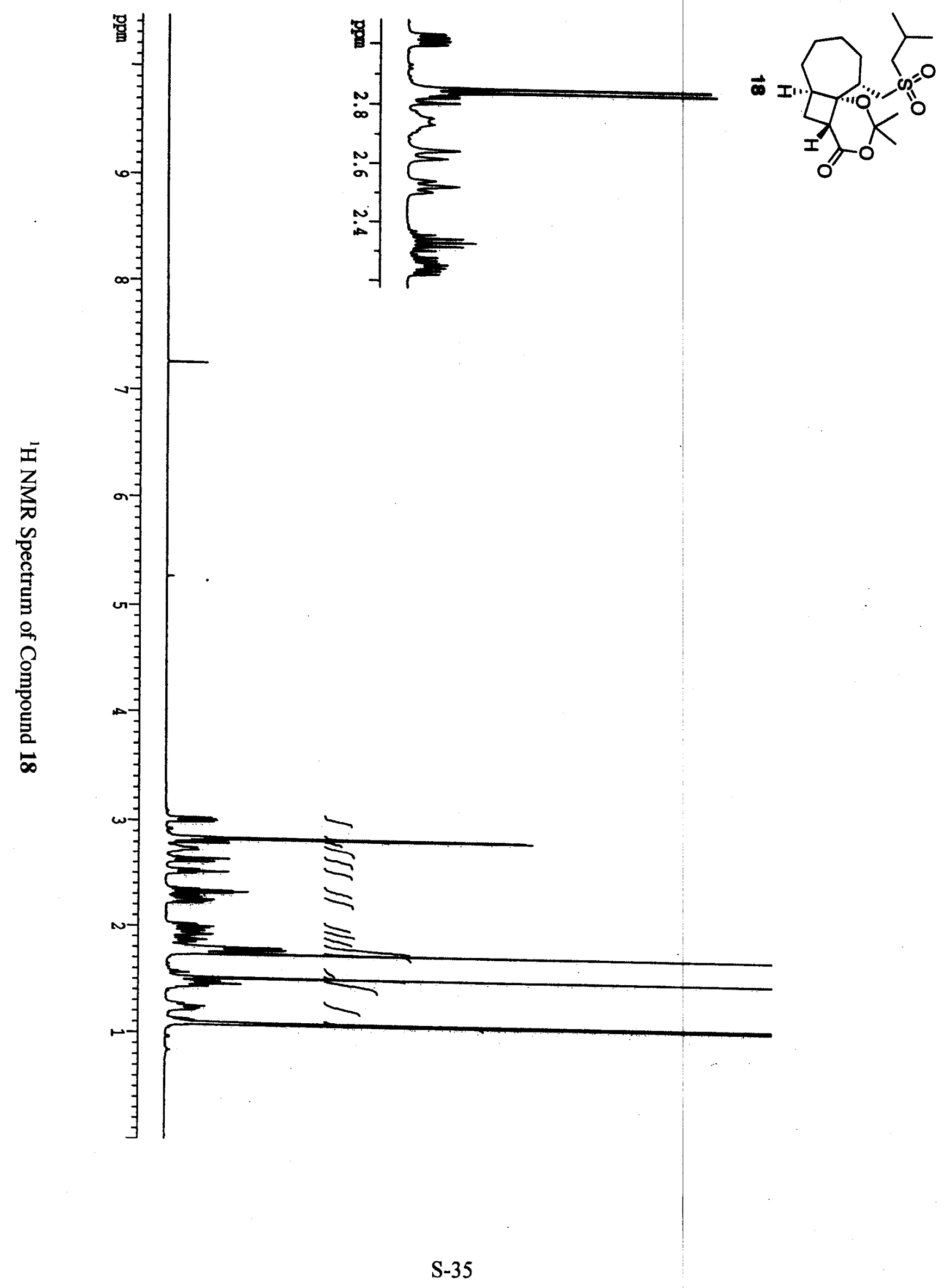



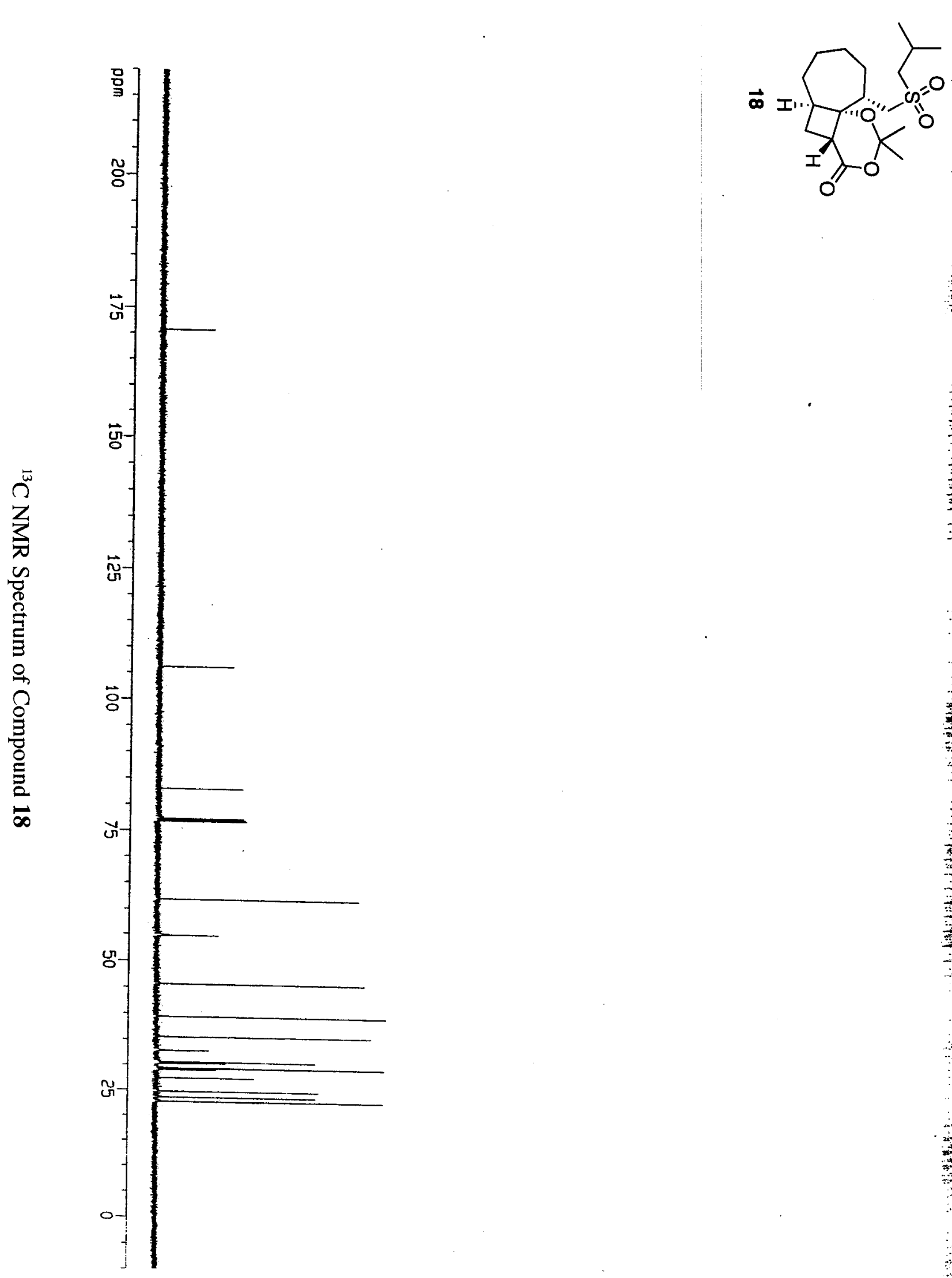


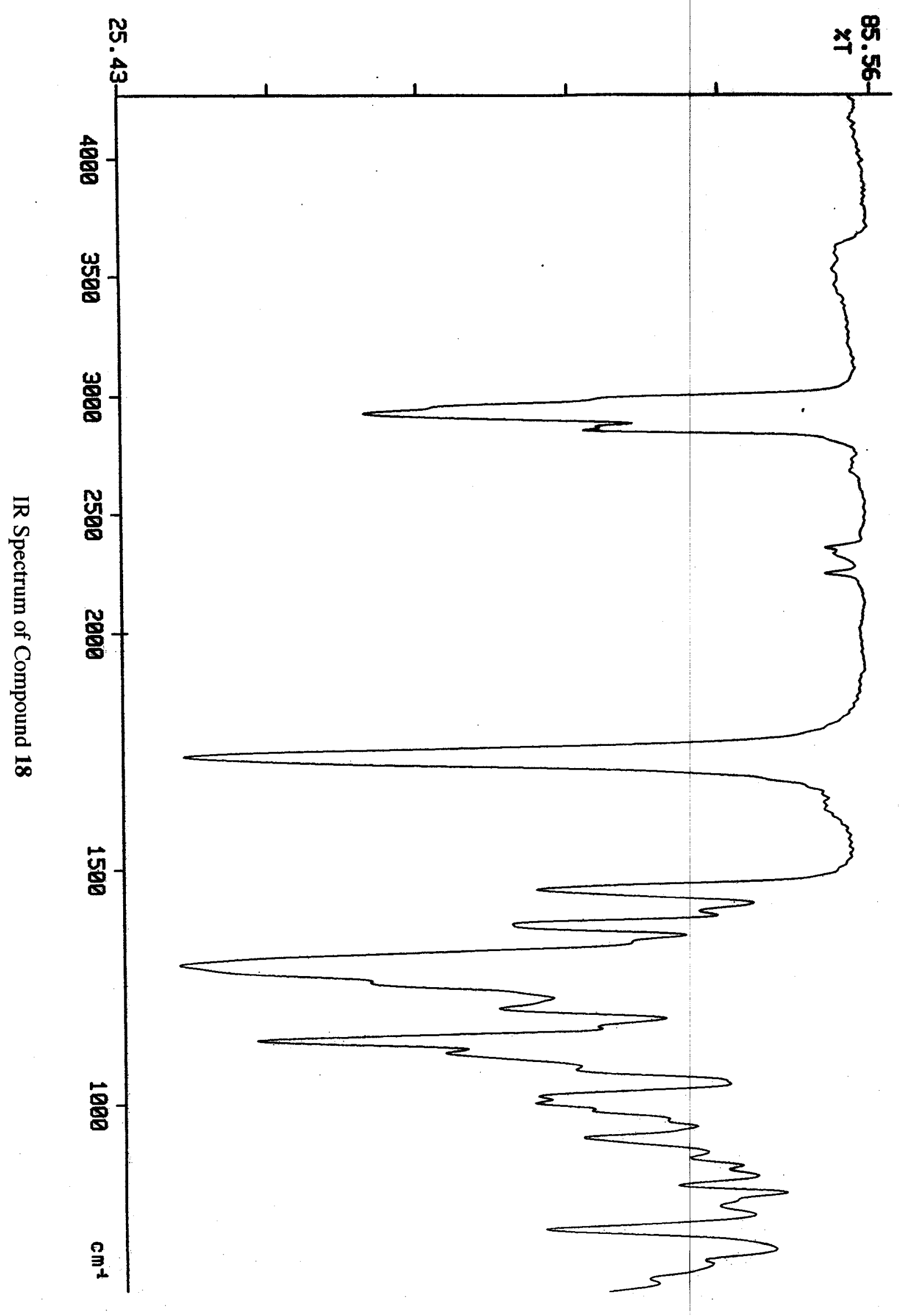

S-37 


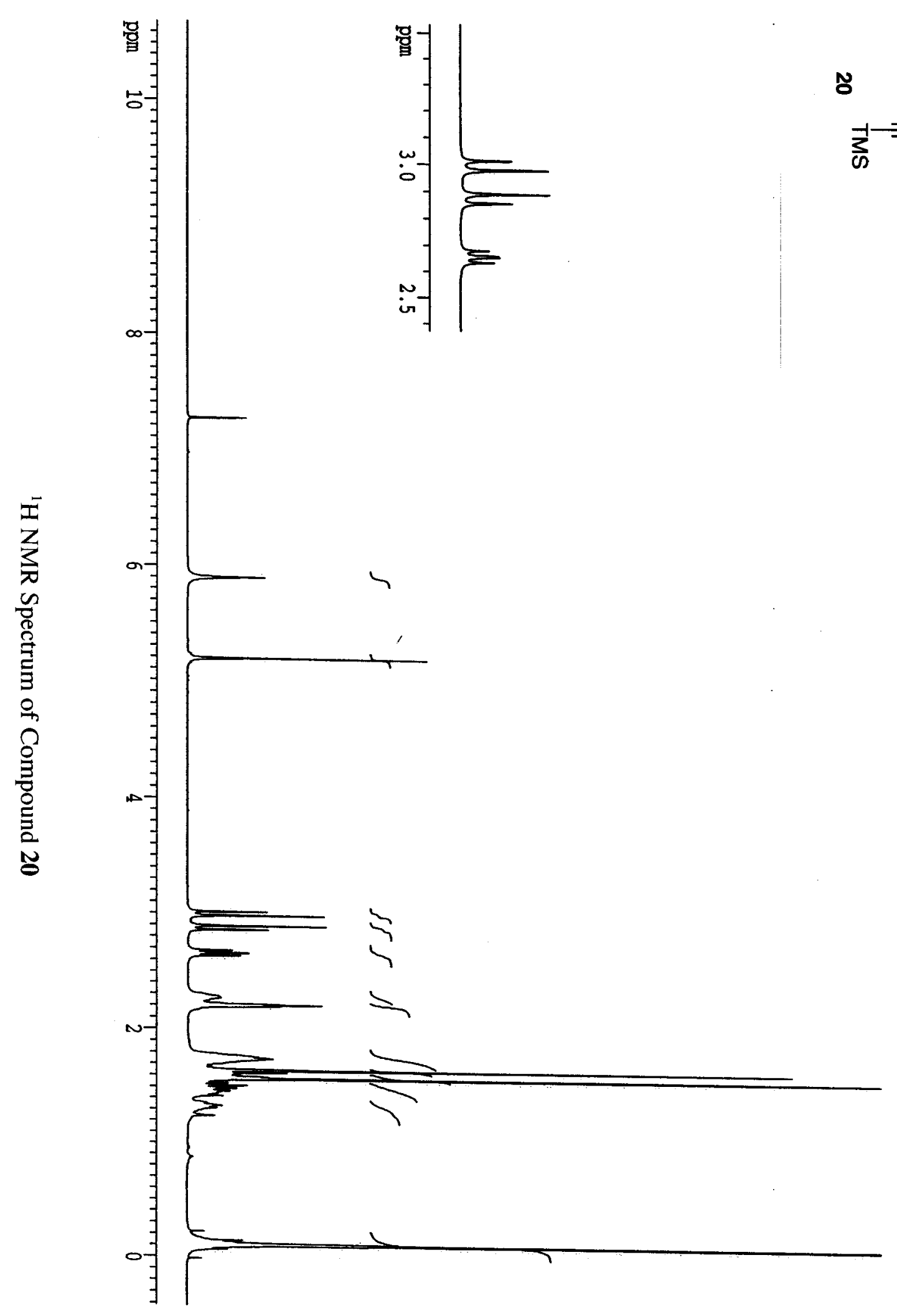




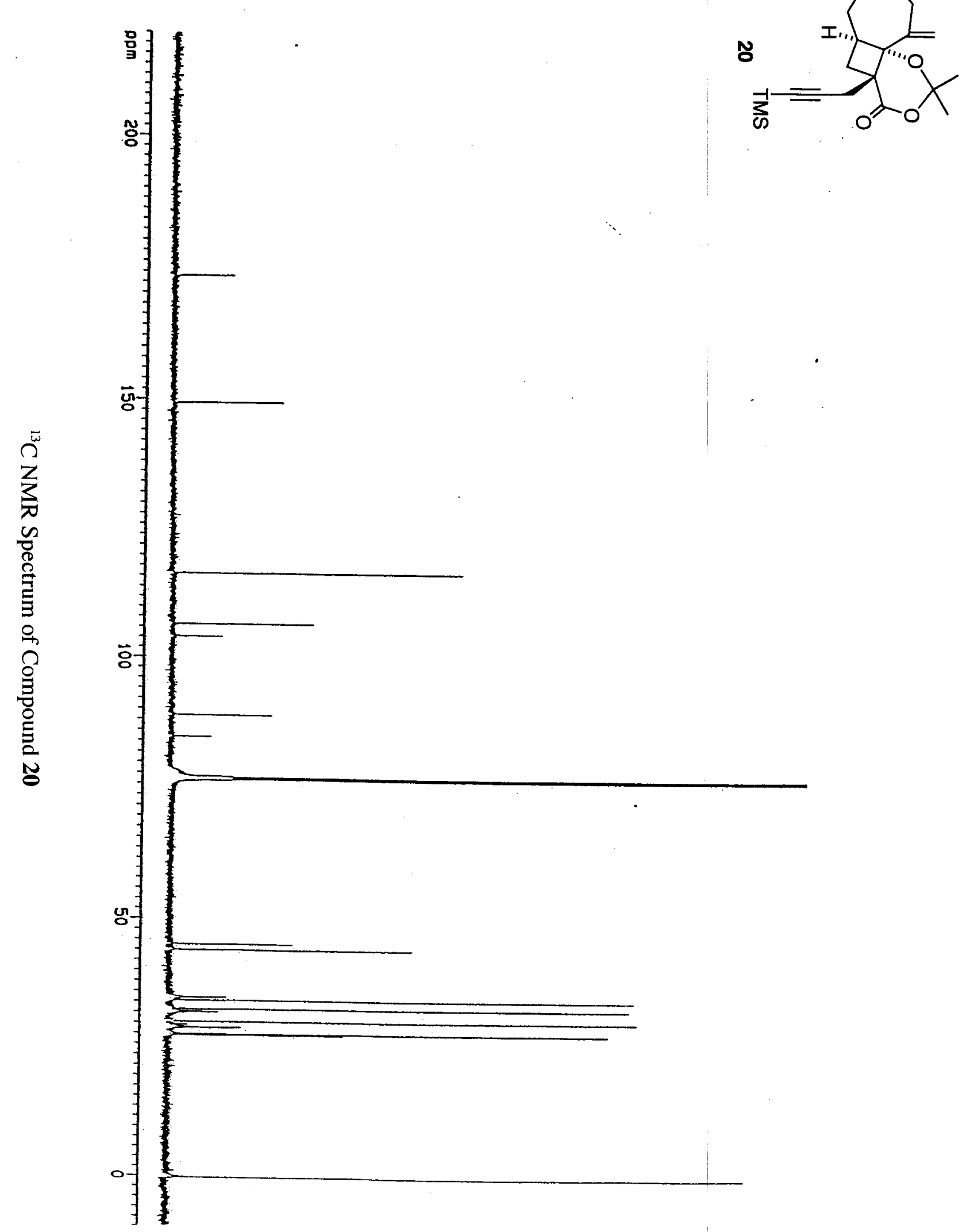

S-39 


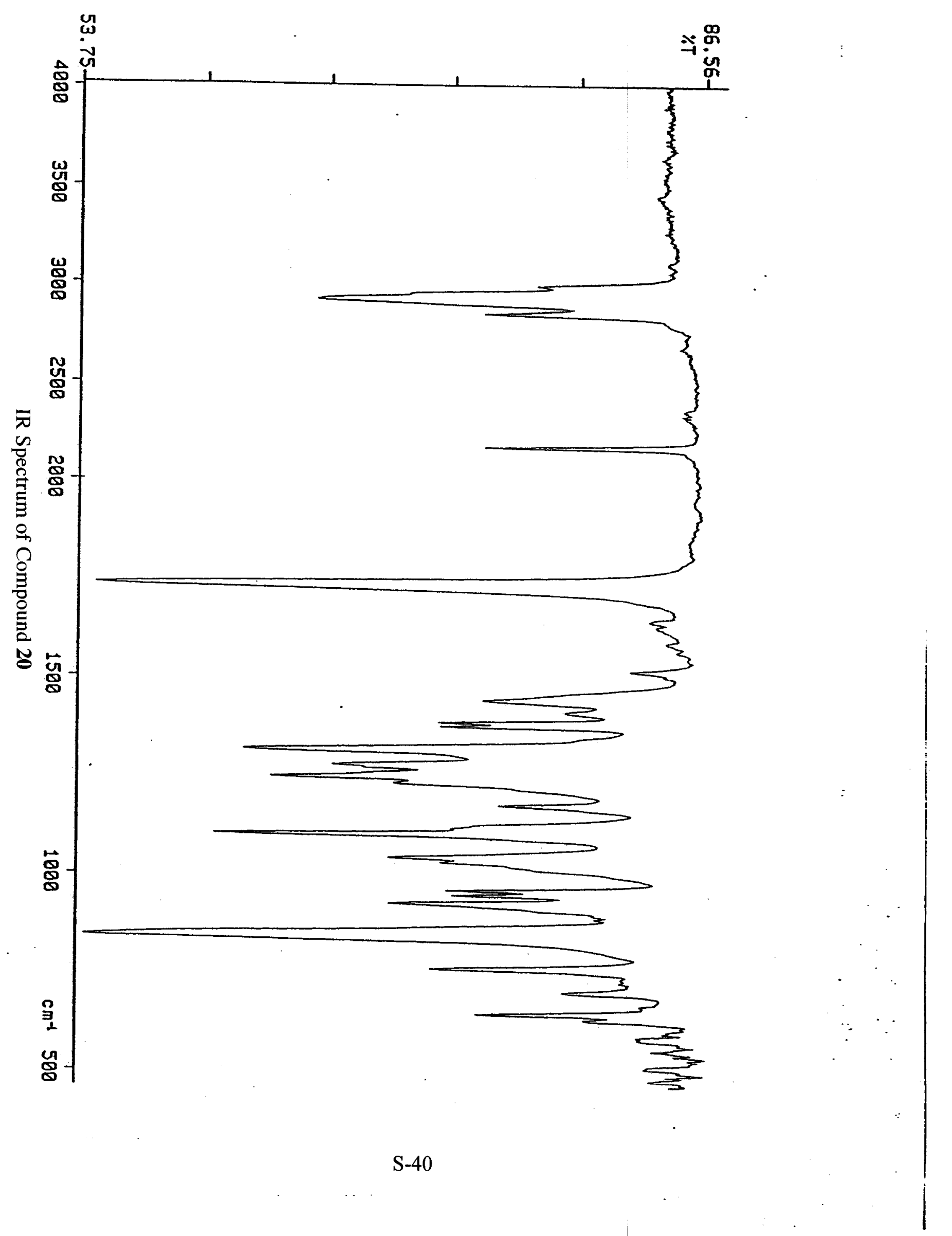




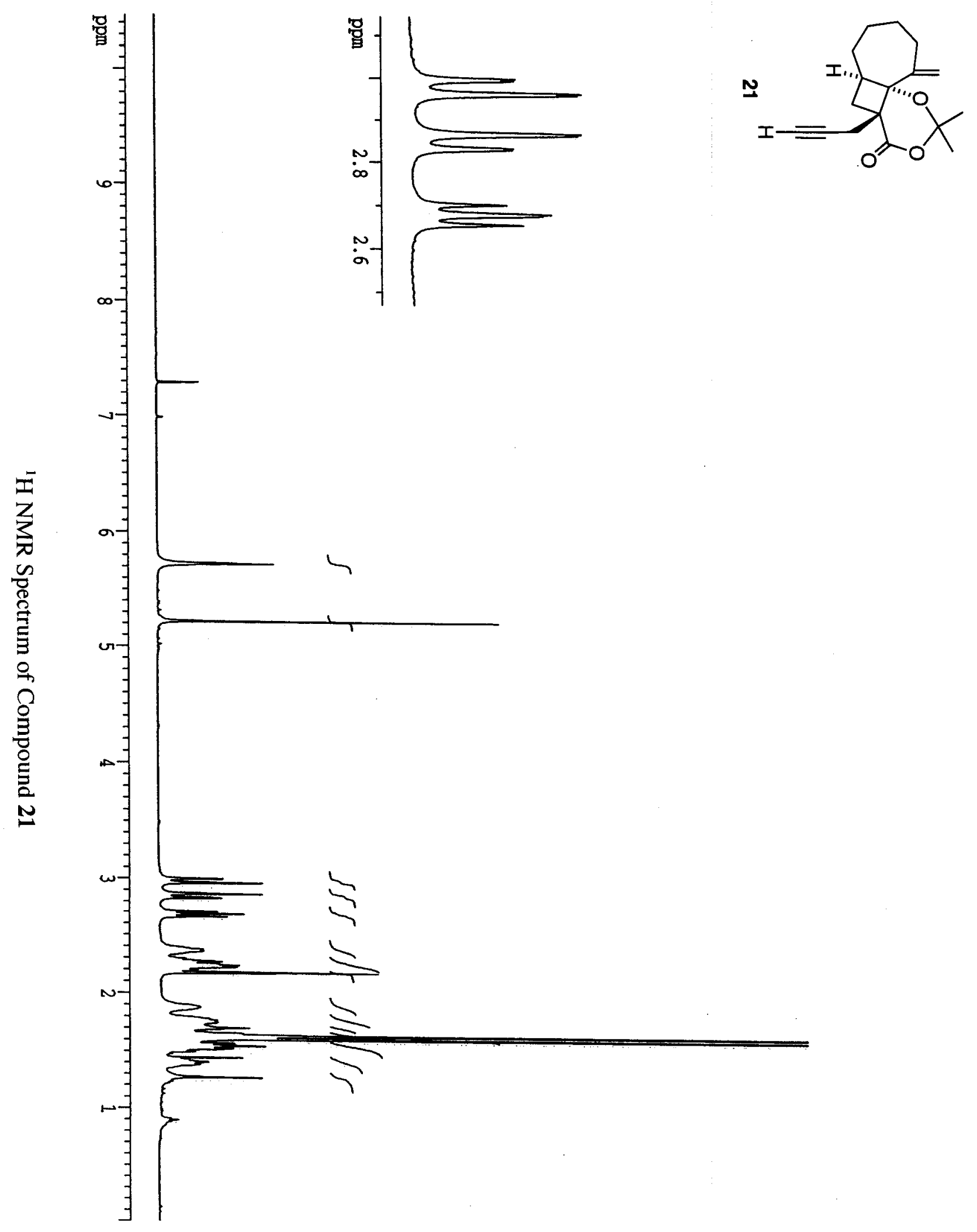




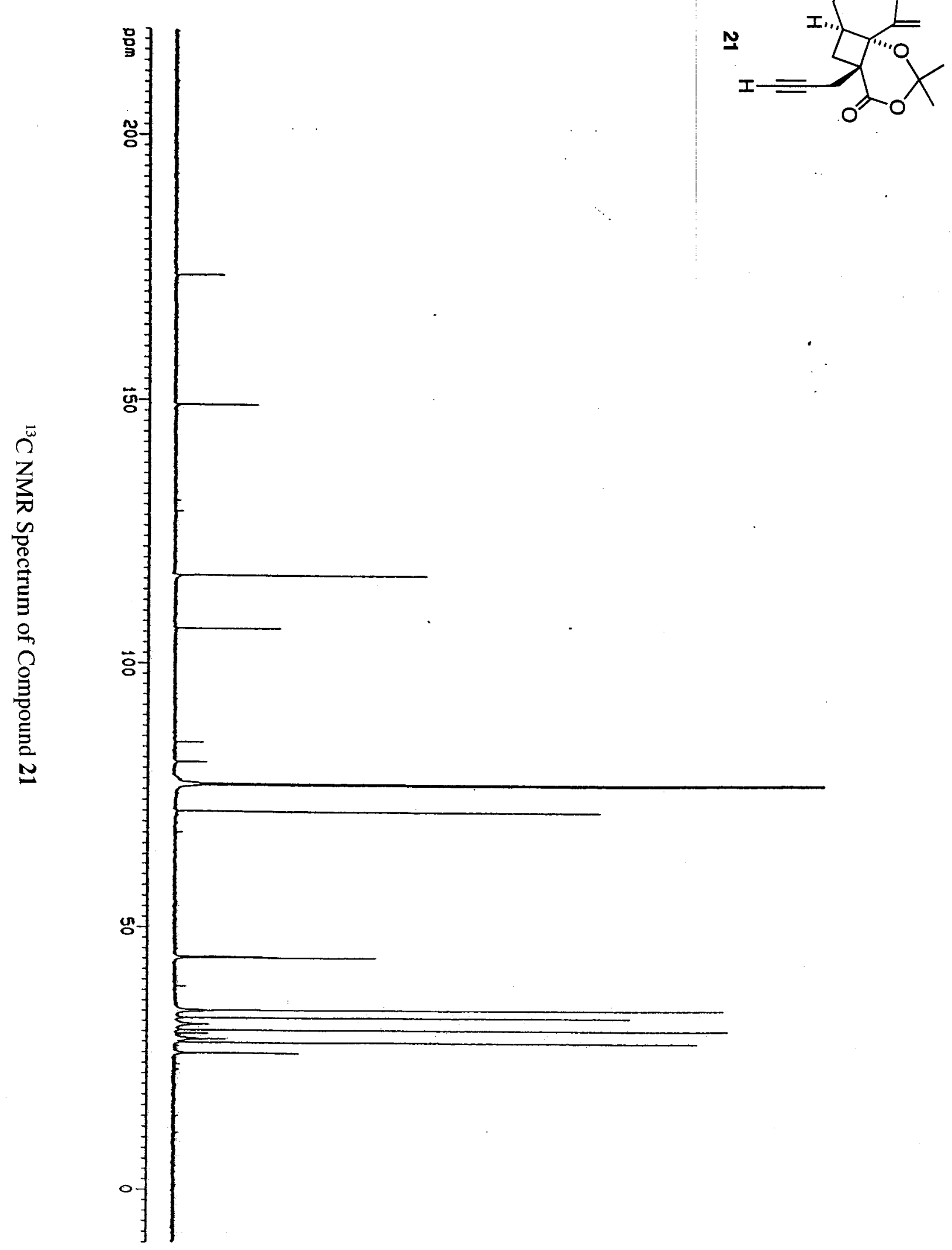




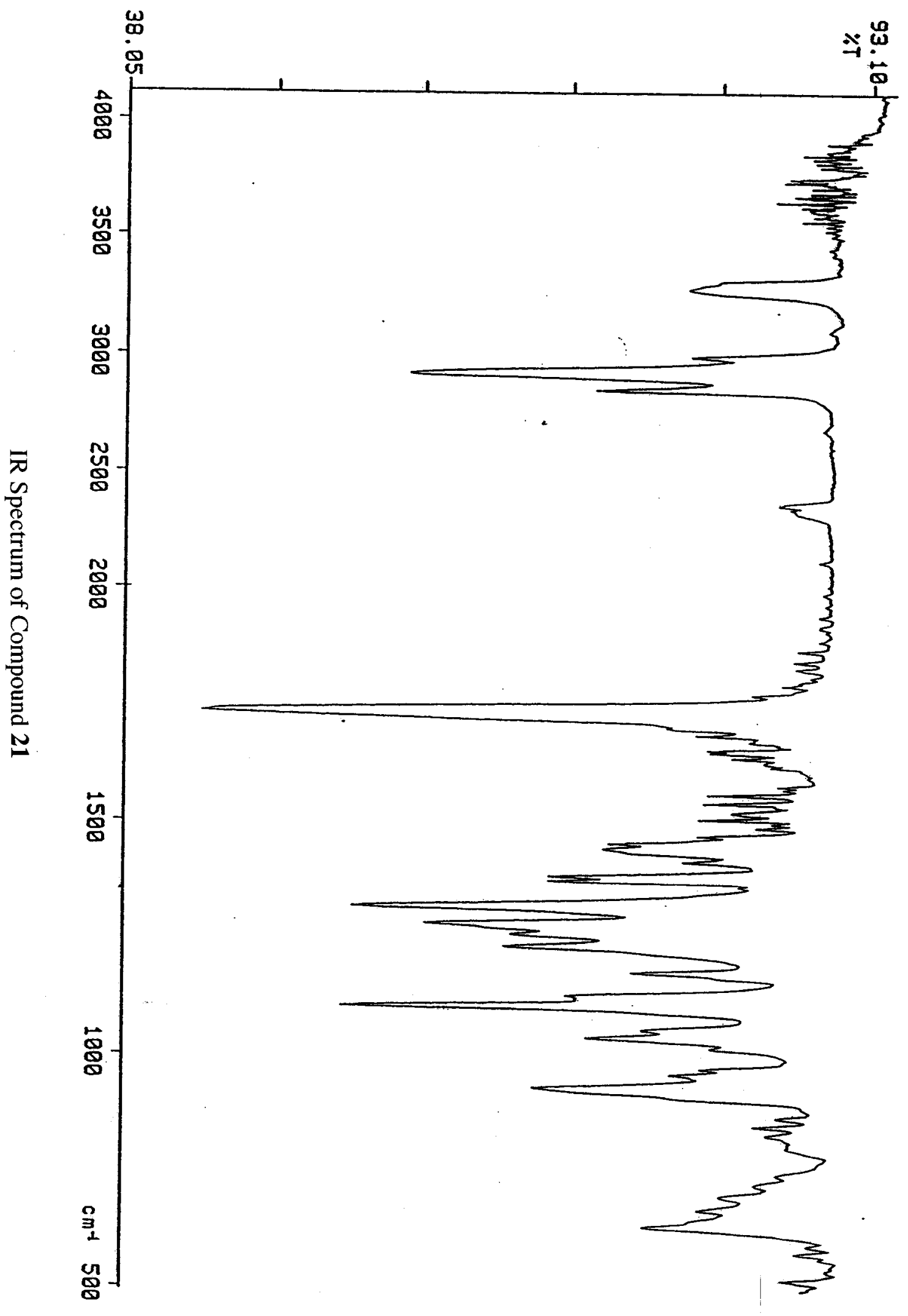




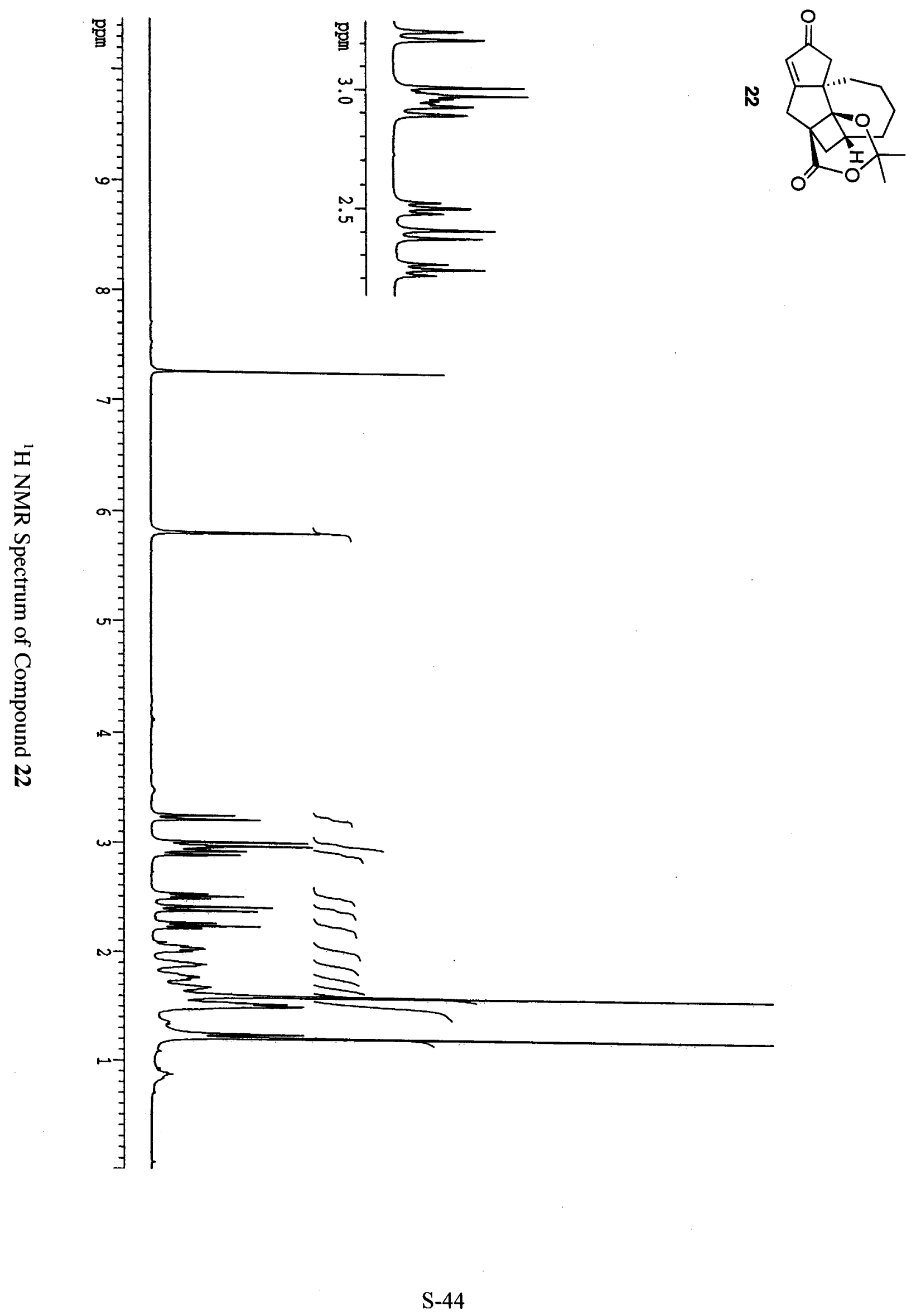




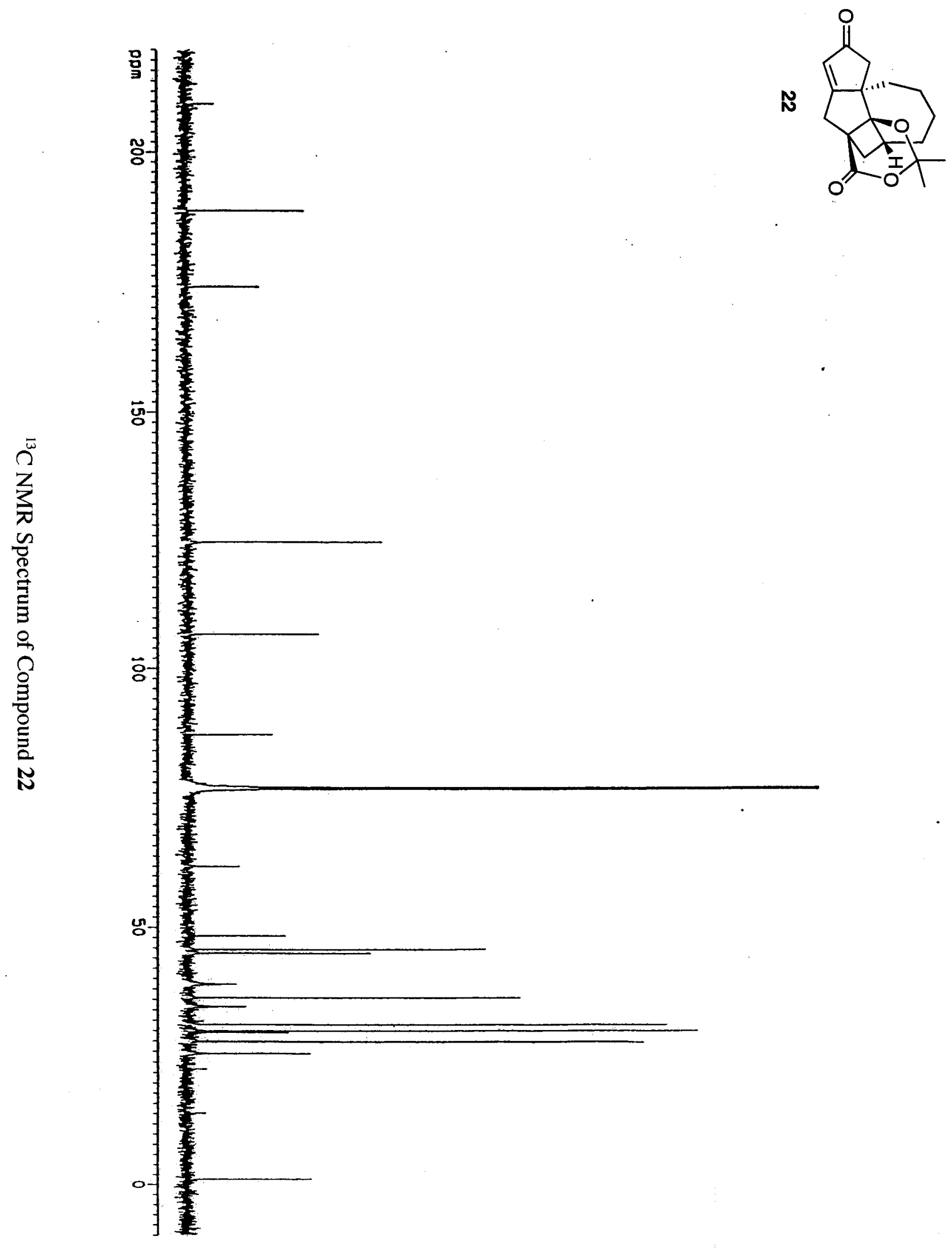




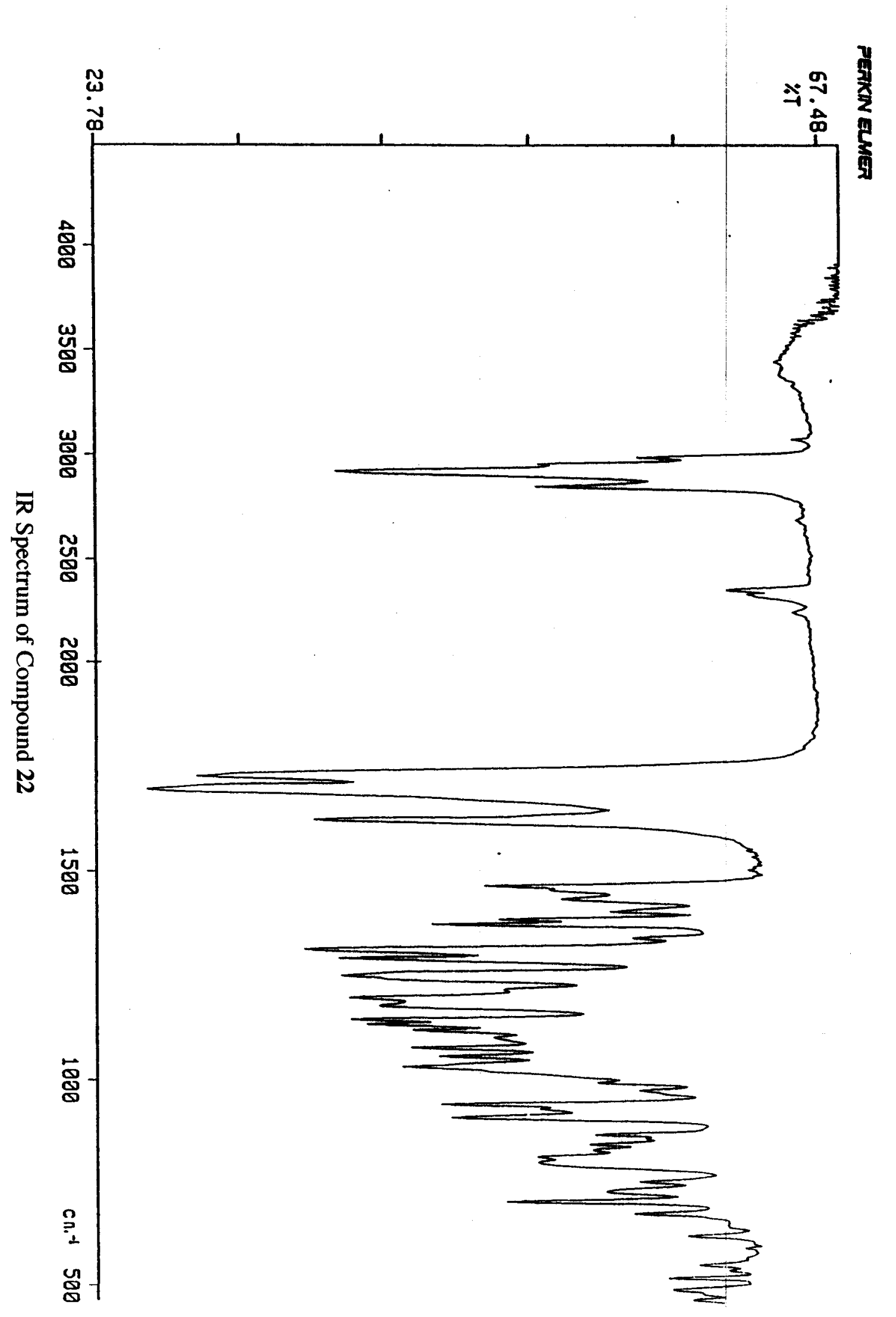




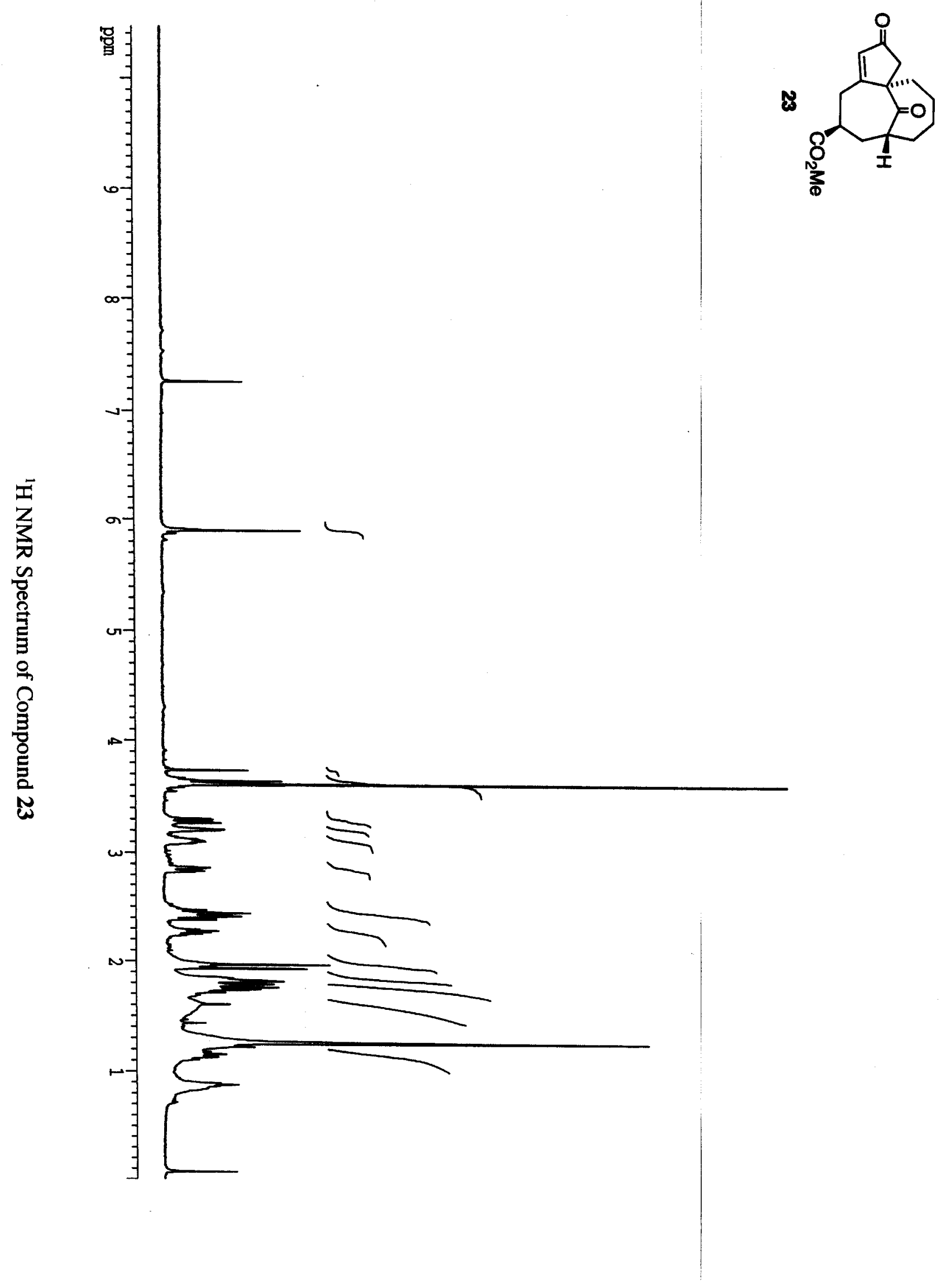



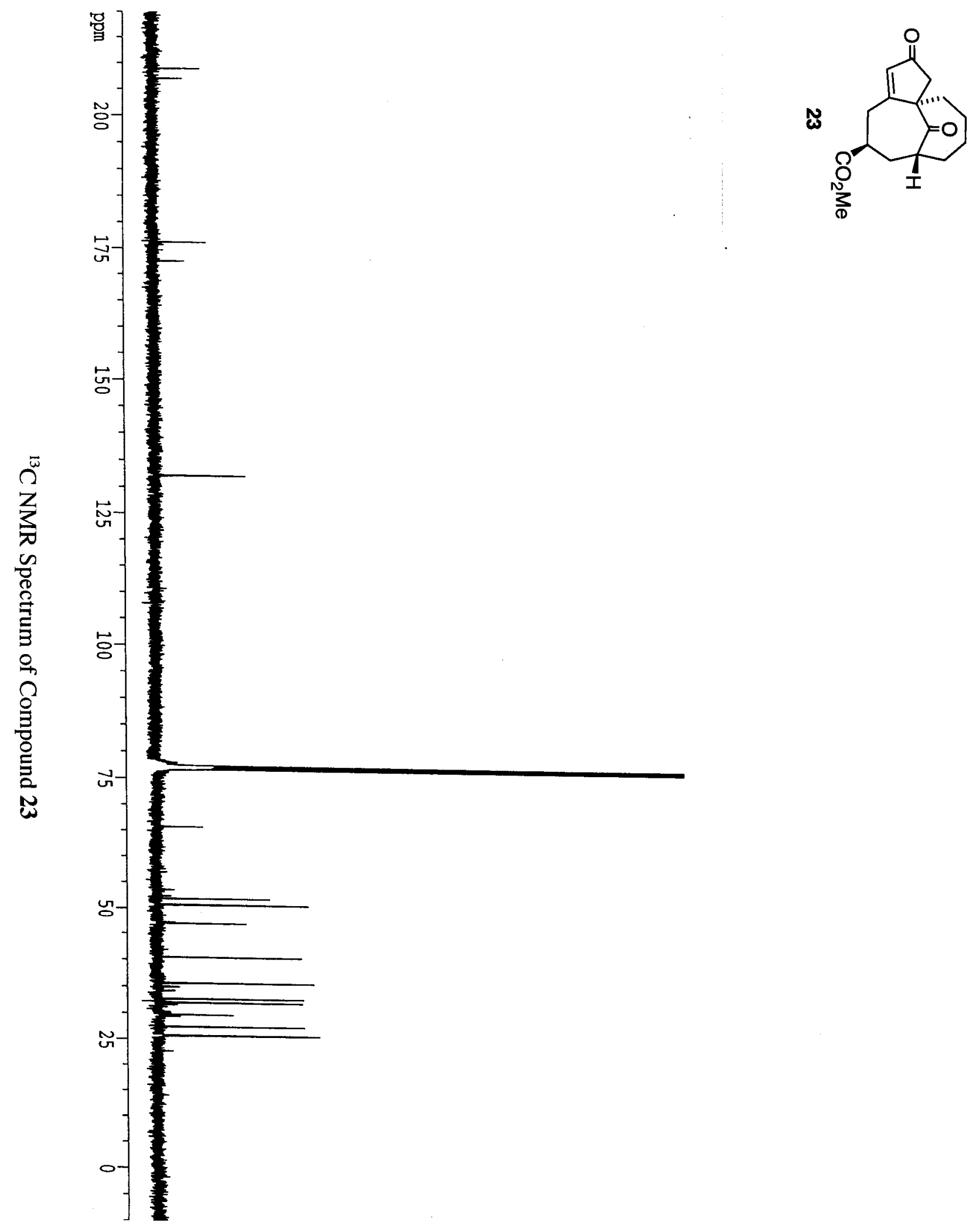


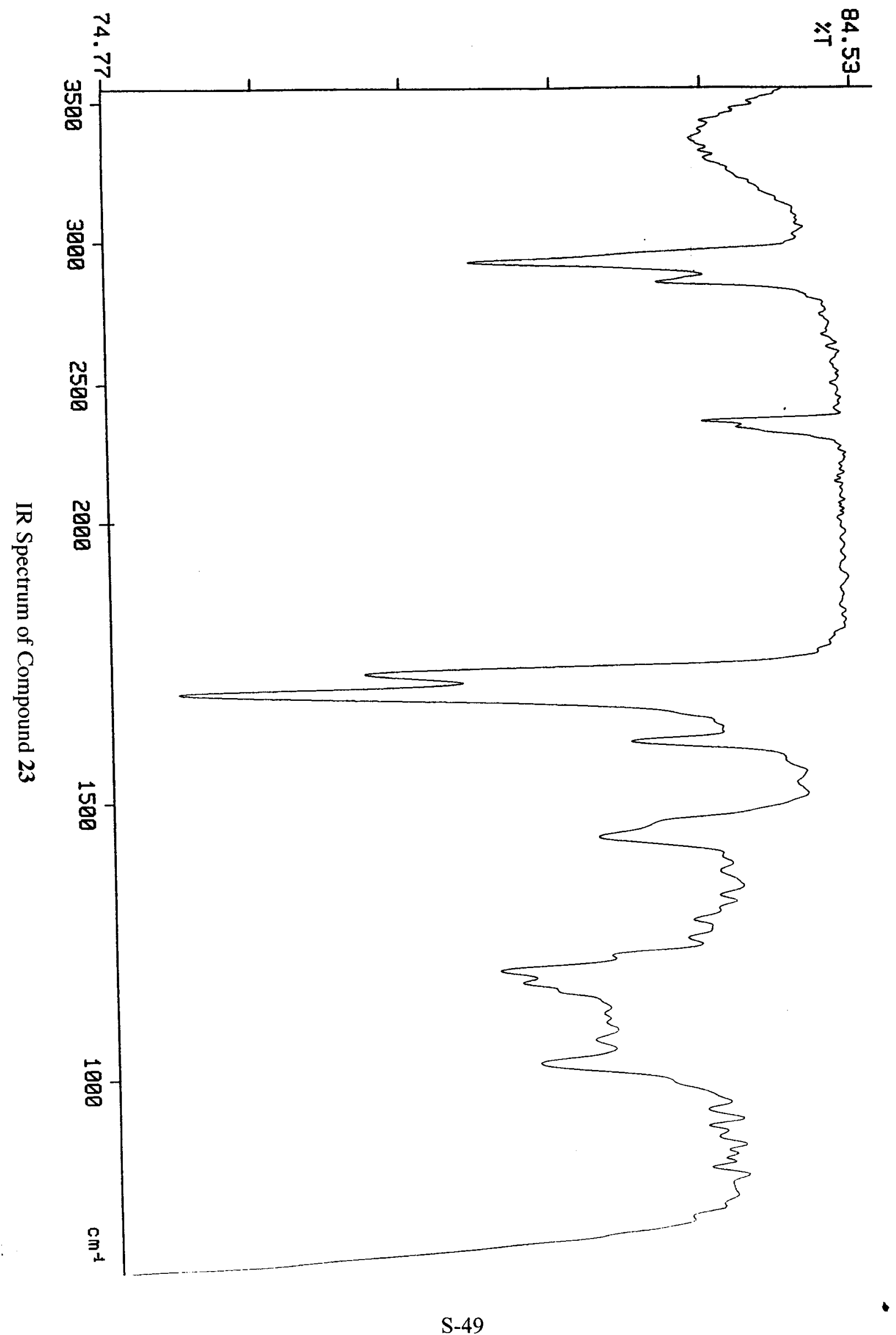




\section{X-ray Structure Determination of Compound 2029}

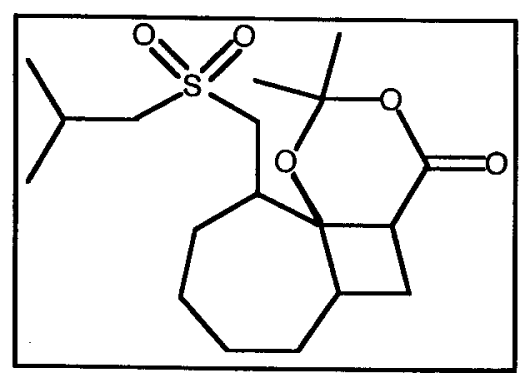

Compound 2029, $\mathrm{C}_{18} \mathrm{H}_{30} \mathrm{SO}_{5}$, crystallizes in the orthorhombic space group Pna2 ${ }_{1}$ (systematic absences hOl: $h=$ odd and Okl: $k+l=0 d d)$ with $a=10.1303(5) \AA, \quad b=25.586(2) \AA, \quad c=7.1022(4) \AA$, $V=1840.9(2) \AA^{3}, Z=4$ and $d_{\text {calc }}=1.293 \mathrm{~g} / \mathrm{cm}^{3}$. X-ray intensity data were collected on a Rigaku Mercury CCD area detector employing graphite-monochromated Mo- $K_{\alpha}$ radiation $(\lambda=0.71069 \AA)$ at a temperature of $143^{\circ} \mathrm{K}$. Preliminary indexing was performed from a series of twelve $0.5^{\circ}$ rotation images with exposures of 30 seconds. A total of 302 rotation images were collected with a crystal to detector distance of $36 \mathrm{~mm}$, a $2 \theta$ swing angle of $-10^{\circ}$, rotation widths of $0.5^{\circ}$ and exposures of 30 seconds: scan no. 1 was a $\phi$-scan from $160^{\circ}$ to $311^{\circ}$ at $\omega=10^{\circ}$ and $\chi=20^{\circ}$. Rotation images were processed using CrystalClear ${ }^{1}$, producing a listing of unaveraged $\mathrm{F}^{2}$ and $\sigma\left(\mathrm{F}^{2}\right)$ values which were then passed to the CrystalStructure ${ }^{2}$ program package for further processing and structure solution on a Dell Pentium III computer. A total of 6916 reflections were measured over the ranges $5.12 \leq 2 \theta \leq 50.7^{\circ},-9 \leq h \leq 12,-30 \leq k \leq 27,-7 \leq 1 \leq 8$ yielding 3096 unique reflections $\left(R_{\text {int }}=0.0137\right)$. The intensity data were corrected for Lorentz and polarization effects and for absorption. using REQAB ${ }^{3}$ (minimum and maximum transmission 0.819, 1.000).

The structure was solved by direct methods (SIR974). Refinement was by full-matrix least squares based on $F^{2}$ using SHELXL-97 ${ }^{5}$. All reflections were used during refinement $\left(F^{2}\right.$ 's that were experimentally negative were replaced by $\left.F^{2}=0\right)$. The weighting scheme used was $w=1 /\left[\sigma^{2}\left(F_{0}^{2}\right)+\right.$ $\left.0.0394 \mathrm{P}^{2}+0.2798 \mathrm{P}\right]$ where $P=\left(F_{0}^{2}+2 F_{c}^{2}\right) / 3$. Non-hydrogen atoms were refined anisotropically and hydrogen atoms were refined using a "riding" model. Refinement converged to $R_{1}=0.0274$ and $w R_{2}=0.0679$ for 3011 reflections for which $F>4 \sigma(F)$ and $R_{1}=0.0284, w R_{2}=0.0687$ and $G O F=1.039$ for all 3096 unique, non-zero reflections and 222 variables $^{6}$. The maximum $\Delta / \sigma$ in the final cycle of least 
squares was 0.000 and the two most prominent peaks in the final difference Fourier were +0.173 and $0.243 \mathrm{e} / \AA^{3}$.

Table 1. lists cell information, data collection parameters, and refinement data. Final positional and equivalent isotropic thermal parameters are given in Table 2. Anisotropic thermal parameters are in Table 3. Tables 4. and 5. list bond distances and bond angles. Figure 1. is an ORTEP ${ }^{7}$ representation of the molecule with $30 \%$ probability thermal ellipsoids displayed.

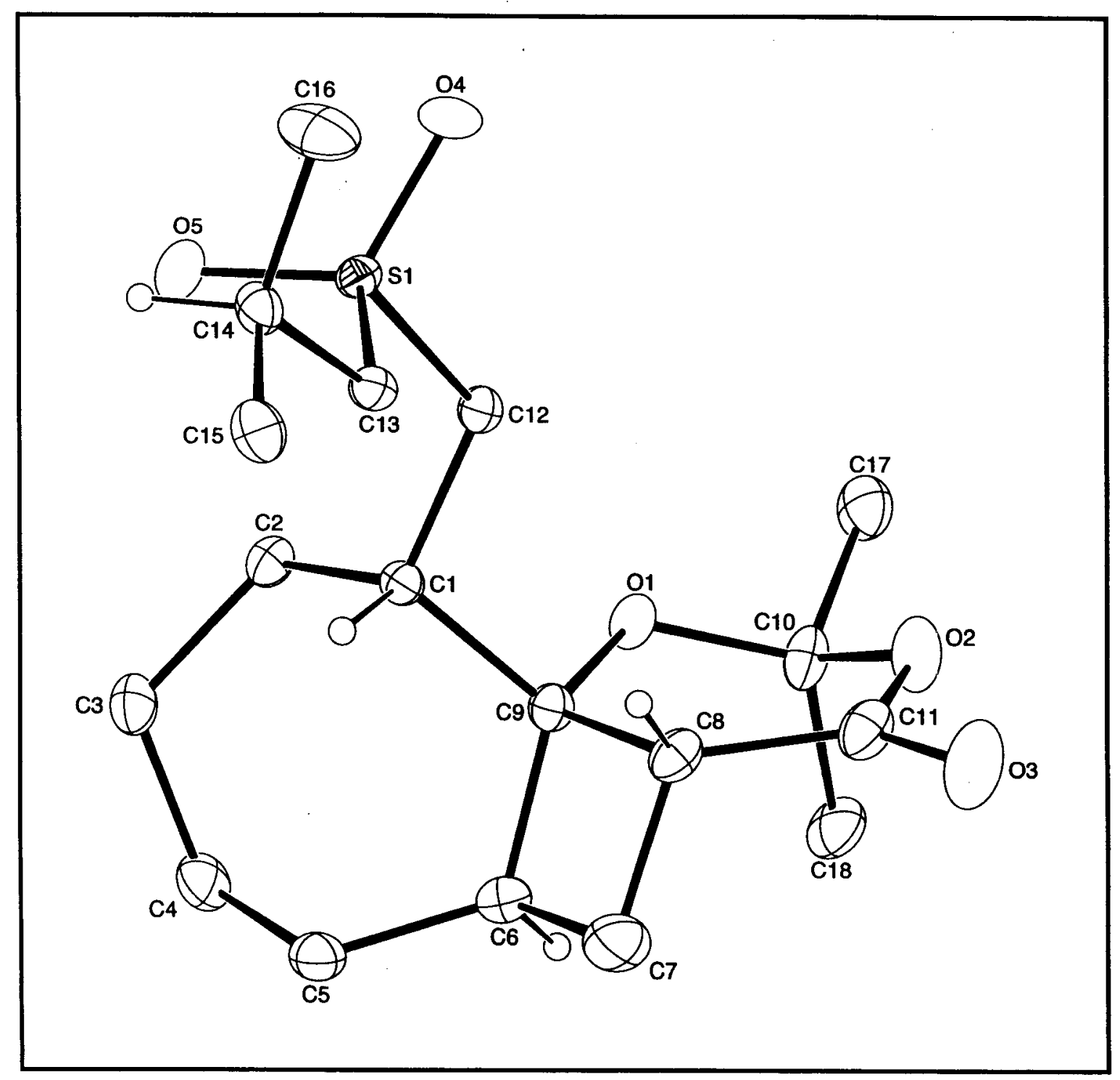

Figure 1. ORTEP drawing of the title compound with $30 \%$ probability thermal ellipsoids. 


\section{References}

1. CrystalClear: Rigaku Corporation, 1999.

2. CrystalStructure: Crystal Structure Analysis Package, Rigaku Corp. Rigaku/MSC (2002).

3. REQAB4: R.A. Jacobsen, (1994). Private Communication.

4. SIIR97: Altomare, A., M. Burla, M. Camalli, G. Cascarano, C. Giacovazzo, A. Guagliardi, A. Moliterni, G. Polidori \& R. Spagna (1999). J. Appl. Cryst., 32, 115-119.

5. SHELXL-97: Program for the Refinement of Crystal Structures, Sheldrick, G.M. (1997), University of Göttingen, Germany.

6. $R_{1}=\Sigma\left\|F_{0}|-| F_{C}\right\| / \Sigma\left|F_{0}\right|$

$$
\begin{aligned}
& w R_{2}=\left\{\sum w\left(F_{o}^{2}-F_{c}^{2}\right)^{2} / \sum w\left(F_{o}^{2}\right)^{2}\right\}^{1 / 2} \\
& G O F=\left\{\sum w\left(F_{o}^{2}-F_{c}^{2}\right)^{2} /(n-p)\right\}^{1 / 2}
\end{aligned}
$$

where $\mathrm{n}=$ the number of reflections and $\mathrm{p}=$ the number of parameters refined.

7. "ORTEP-II: A Fortran Thermal Ellipsoid Plot Program for Crystal Structure Illustrations". C.K. Johnson (1976) ORNL-5138. 
Table 1. Summary of Structure Determination of Compound 2029

Formula:

Formula weight:

Crystal class:

Space group:

Z

Cell constants:

a

b

c

V

$\mu$

crystal size, $\mathrm{mm}$

$D_{\text {calc }}$

$\mathrm{F}(000)$

Radiation:

$2 \theta$ range

hkl collected:

No. reflections measured:

No. unique reflections:

No. observed reflections

No. reflections used in refinement

No. parameters

$R$ indices $(F>4 \sigma)$

$R$ indices (all data)

GOF:

Final Difference Peaks, $\mathrm{e} / \AA^{3}$
$\mathrm{C}_{18} \mathrm{H}_{30} \mathrm{SO}_{5}$

358.48

orthorhombic

$\mathrm{Pna2}_{1}$ (\#33)

4

$10.1303(5) \AA$

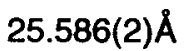

$7.1022(4) \AA$

$1840.9(2) \AA^{3}$

$2.00 \mathrm{~cm}^{-1}$

$0.35 \times 0.27 \times 0.22$

$1.293 \mathrm{~g} / \mathrm{cm}^{3}$

776

Mo-K $K_{\alpha}(\lambda=0.71069 \AA)$

$5.12-50.7^{\circ}$

$-9 \leq h \leq 12 ;-30 \leq k \leq 27 ;-7 \leq 1 \leq 8$

6916

$3096\left(R_{\text {int }}=0.0137\right)$

3011 ( $F>4 \sigma$ )

3096

222

$R_{1}=0.0274$

$w R_{2}=0.0679$

$R_{1}=0.0284$

$w R_{2}=0.0687$

1.039

$+0.173,-0.243$ 
Table 2. Refined Positional Parameters for Compound 2029

\begin{tabular}{|c|c|c|c|c|}
\hline Atom & $x$ & $y$ & z & $U_{e q}, \AA^{2}$ \\
\hline S1 & $0.29685(3)$ & $0.266736(13)$ & $0.74888(7)$ & $0.02196(11)$ \\
\hline $\mathrm{C} 1$ & $0.3693(2)$ & $0.16552(6)$ & $0.8716(2)$ & $0.0204(3)$ \\
\hline $\mathrm{H} 1$ & 0.4506 & 0.1755 & 0.8060 & 0.027 \\
\hline $\mathrm{C} 2$ & $0.3905(2)$ & $0.17253(6)$ & $1.0849(2)$ & $0.0260(4)$ \\
\hline $\mathrm{H} 2 \mathrm{a}$ & 0.3731 & 0.2088 & 1.1165 & 0.035 \\
\hline $\mathrm{H} 2 \mathrm{~b}$ & 0.3256 & 0.1514 & 1.1506 & 0.035 \\
\hline C3 & $0.5267(2)$ & $0.15825(7)$ & $1.1592(3)$ & $0.0316(4)$ \\
\hline H3a & 0.5916 & 0.1796 & 1.0948 & 0.042 \\
\hline H3b & 0.5304 & 0.1671 & 1.2919 & 0.042 \\
\hline $\mathrm{C} 4$ & $0.5659(2)$ & $0.10104(7)$ & $1.1369(3)$ & $0.0364(4)$ \\
\hline $\mathrm{H} 4 \mathrm{a}$ & 0.6496 & 0.0953 & 1.1999 & 0.048 \\
\hline $\mathrm{H} 4 \mathrm{~b}$ & 0.5003 & 0.0793 & 1.1985 & 0.048 \\
\hline C5 & $0.5784(2)$ & $0.08403(6)$ & $0.9324(3)$ & $0.0315(4)$ \\
\hline H5a & 0.6156 & 0.1126 & 0.8600 & 0.042 \\
\hline $\mathrm{H} 5 \mathrm{~b}$ & 0.6396 & 0.0549 & 0.9253 & 0.042 \\
\hline C6 & $0.4491(2)$ & $0.06788(7)$ & $0.8438(3)$ & $0.0309(4)$ \\
\hline $\mathrm{H} 6$ & 0.4150 & 0.0365 & 0.9065 & 0.041 \\
\hline $\mathrm{C} 7$ & $0.4502(2)$ & $0.05960(9)$ & $0.6305(3)$ & $0.0517(6)$ \\
\hline $\mathrm{H} 7 \mathrm{a}$ & 0.4321 & 0.0238 & 0.5935 & 0.069 \\
\hline $\mathrm{H} 7 \mathrm{~b}$ & 0.5300 & 0.0723 & 0.5702 & 0.069 \\
\hline C8 & $0.3306(2)$ & $0.09656(7)$ & $0.6057(2)$ & $0.0291(4)$ \\
\hline H8 & 0.3543 & 0.1273 & 0.5307 & 0.039 \\
\hline C9 & $0.3351(2)$ & $0.10895(6)$ & $0.8192(2)$ & $0.0227(3)$ \\
\hline C10 & $0.1260(2)$ & $0.06017(6)$ & $0.8522(3)$ & $0.0299(4)$ \\
\hline C11 & $0.2082(2)$ & $0.07076(7)$ & $0.5305(3)$ & $0.0330(4)$ \\
\hline $\mathrm{C} 12$ & $0.2563(2)$ & $0.20061(6)$ & $0.8015(2)$ & $0.0238(4)$ \\
\hline $\mathrm{H} 12 \mathrm{a}$ & 0.1875 & 0.2007 & 0.8965 & 0.032 \\
\hline $\mathrm{H} 12 \mathrm{~b}$ & 0.2195 & 0.1849 & 0.6889 & 0.032 \\
\hline C13 & $0.3952(2)$ & $0.26282(6)$ & $0.5417(3)$ & $0.0240(4)$ \\
\hline H13a & 0.4658 & 0.2379 & 0.5627 & 0.032 \\
\hline $\mathrm{H} 13 \mathrm{~b}$ & 0.3411 & 0.2497 & 0.4394 & 0.032 \\
\hline C14 & $0.4556(2)$ & $0.31521(6)$ & $0.4826(3)$ & $0.0270(4)$ \\
\hline $\mathrm{H} 14$ & 0.5023 & 0.3301 & 0.5909 & 0.036 \\
\hline C15 & $0.5554(2)$ & $0.30514(7)$ & $0.3262(3)$ & $0.0342(4)$ \\
\hline H15a & 0.6187 & 0.2797 & 0.3679 & 0.051 \\
\hline H15b & 0.5999 & 0.3371 & 0.2955 & 0.051 \\
\hline $\mathrm{H} 15 \mathrm{c}$ & 0.5104 & 0.2922 & 0.2168 & 0.051 \\
\hline C16 & $0.3532(2)$ & $0.35443(8)$ & $0.4156(4)$ & $0.0504(6)$ \\
\hline $\mathrm{H} 16 \mathrm{a}$ & 0.3026 & 0.3394 & 0.3149 & 0.076 \\
\hline $\mathrm{H} 16 \mathrm{~b}$ & 0.3968 & 0.3854 & 0.3714 & 0.076 \\
\hline $\mathrm{H} 16 \mathrm{c}$ & 0.2956 & 0.3633 & 0.5180 & 0.076 \\
\hline C17 & $-0.0055(2)$ & $0.07332(7)$ & $0.9376(3)$ & $0.0383(5)$ \\
\hline $\mathrm{H} 17 \mathrm{a}$ & 0.0010 & 0.0718 & 1.0724 & 0.057 \\
\hline $\mathrm{H} 17 \mathrm{~b}$ & -0.0704 & 0.0487 & 0.8951 & 0.057 \\
\hline $\mathrm{H} 17 \mathrm{c}$ & -0.0310 & 0.1079 & 0.9000 & 0.057 \\
\hline C18 & $0.1688(2)$ & $0.00489(7)$ & $0.9002(3)$ & $0.0405(5)$ \\
\hline $\mathrm{H} 18 \mathrm{a}$ & 0.2400 & -0.0053 & 0.8189 & 0.061 \\
\hline $\mathrm{H} 18 \mathrm{~b}$ & 0.0958 & -0.0185 & 0.8831 & 0.061 \\
\hline
\end{tabular}




\begin{tabular}{|lllll|}
\hline $\mathrm{H} 18 \mathrm{c}$ & 0.1977 & 0.0036 & 1.0288 & 0.061 \\
$\mathrm{O} 1$ & $0.21301(10)$ & $0.09944(4)$ & $0.9148(2)$ & $0.0248(3)$ \\
$\mathrm{O} 2$ & $0.10652(14)$ & $0.06315(5)$ & $0.6477(2)$ & $0.0390(3)$ \\
$\mathrm{O} 3$ & $0.1961(2)$ & $0.05914(7)$ & $0.3682(2)$ & $0.0561(4)$ \\
$\mathrm{O} 4$ & $0.17399(11)$ & $0.29230(4)$ & $0.7034(2)$ & $0.0334(3)$ \\
$\mathrm{O} 5$ & $0.37575(12)$ & $0.28864(4)$ & $0.8974(2)$ & $0.0338(3)$ \\
\hline \multicolumn{2}{|r}{$U_{\mathrm{eq}}={ }^{1 / 3}\left[\mathrm{U}_{11}\left(\mathrm{aa}^{*}\right)^{2}+\mathrm{U}_{22}\left(\mathrm{bb}^{*}\right)^{2}+\mathrm{U}_{33}\left(\mathrm{cc}^{*}\right)^{2}+2 \mathrm{U}_{12} \mathrm{aa}^{*} \mathrm{bb}^{*} \cos \gamma+2 \mathrm{U}_{13} \mathrm{aa}^{*} \mathrm{cc}^{*} \cos \beta+2 \mathrm{U}_{23} \mathrm{bb}^{*} \mathrm{cc} c^{*} \cos \alpha\right]$} \\
\hline
\end{tabular}


Table 3. Refined Thermal Parameters (U's) for Compound 2029

\begin{tabular}{|c|c|c|c|c|c|c|}
\hline Atom & $U_{11}$ & $\mathrm{U}_{22}$ & $\mathrm{U}_{33}$ & $U_{23}$ & $U_{13}$ & $U_{12}$ \\
\hline S1 & $0.0220(2)$ & $0.0175(2)$ & $0.0264(2)$ & $-0.0020(2)$ & $0.0010(2)$ & $0.00050(13)$ \\
\hline C1 & $0.0194(7)$ & $0.0196(7)$ & $0.0222(9)$ & $0.0017(6)$ & $0.0001(6)$ & $-0.0004(6)$ \\
\hline $\mathrm{C} 2$ & $0.0303(8)$ & $0.0245(8)$ & $0.0233(9)$ & $-0.0038(7)$ & $-0.0005(7)$ & $-0.0010(6)$ \\
\hline C3 & $0.0339(9)$ & $0.0334(9)$ & $0.0274(10)$ & $-0.0008(7)$ & $-0.0077(7)$ & $-0.0050(7)$ \\
\hline C4 & $0.0360(10)$ & $0.0341(9)$ & $0.0390(11)$ & $0.0048(8)$ & $-0.0145(8)$ & $0.0025(8)$ \\
\hline C5 & $0.0277(8)$ & $0.0245(8)$ & $0.0424(12)$ & $0.0019(8)$ & $0.0004(8)$ & $0.0028(7)$ \\
\hline C6 & $0.0326(10)$ & $0.0241(8)$ & $0.0359(11)$ & $-0.0024(7)$ & $-0.0020(8)$ & $0.0049(7)$ \\
\hline $\mathrm{C7}$ & $0.0505(13)$ & $0.0616(14)$ & $0.0429(14)$ & $-0.0243(11)$ & $-0.0063(10)$ & $0.0217(11)$ \\
\hline C8 & $0.0399(10)$ & $0.0260(8)$ & $0.0214(9)$ & $-0.0018(7)$ & $0.0015(8)$ & $-0.0011(7)$ \\
\hline C9 & $0.0235(8)$ & $0.0234(8)$ & $0.0212(8)$ & $-0.0003(7)$ & $0.0002(7)$ & $-0.0006(6)$ \\
\hline C10 & $0.0361(10)$ & $0.0268(9)$ & $0.0267(10)$ & $-0.0005(7)$ & $-0.0041(8)$ & $-0.0109(7)$ \\
\hline C11 & $0.0478(11)$ & $0.0266(9)$ & $0.0247(11)$ & $0.0006(8)$ & $-0.0050(8)$ & $-0.0031(7)$ \\
\hline C12 & $0.0219(7)$ & $0.0209(7)$ & $0.0285(10)$ & $0.0000(6)$ & $-0.0007(7)$ & $-0.0024(6)$ \\
\hline C13 & $0.0214(8)$ & $0.0235(8)$ & $0.0272(10)$ & $-0.0009(7)$ & $0.0010(7)$ & $0.0017(6)$ \\
\hline C14 & $0.0234(8)$ & $0.0244(8)$ & $0.0331(10)$ & $0.0042(7)$ & $-0.0036(7)$ & $-0.0036(6)$ \\
\hline C15 & $0.0294(9)$ & $0.0412(10)$ & $0.0320(10)$ & $0.0050(8)$ & $0.0004(7)$ & $-0.0065(7)$ \\
\hline C16 & $0.0410(11)$ & $0.0366(10)$ & $0.074(2)$ & $0.0256(11)$ & $0.0067(12)$ & $0.0067(9)$ \\
\hline C17 & $0.0343(10)$ & $0.0350(9)$ & $0.0456(13)$ & $0.0007(8)$ & $0.0011(9)$ & $-0.0109(8)$ \\
\hline $\mathrm{C} 18$ & $0.0482(11)$ & $0.0231(8)$ & $0.0502(13)$ & $0.0006(9)$ & $-0.0012(10)$ & $-0.0094(8)$ \\
\hline 01 & $0.0293(6)$ & $0.0217(5)$ & $0.0233(7)$ & $-0.0008(5)$ & $0.0026(5)$ & $-0.0069(4)$ \\
\hline 02 & $0.0439(8)$ & $0.0460(8$ & $0.0270(7)$ & $-0.0008(6)$ & $-0.0079(6)$ & $-0.0121(6)$ \\
\hline 03 & $0.0767(12)$ & $0.0673(10)$ & $0.0243(9)$ & $-0.0094(7)$ & $-0.0074(7)$ & $-0.0197(8)$ \\
\hline O4 & $0.0259(6)$ & $0.0278(6)$ & $0.0464(9)$ & $0.0033(5)$ & $0.0047(6)$ & $0.0088(5)$ \\
\hline O5 & $0.0423(7)$ & $0.0270(6)$ & $0.0320(7)$ & $-0.0048(5)$ & $-0.0044(6)$ & $-0.0065(5)$ \\
\hline
\end{tabular}


Table 4. Bond Distances in Compound 2029, $\AA$

\begin{tabular}{|llllll|}
\hline $\mathrm{S} 1-\mathrm{O} 5$ & $1.4373(13)$ & $\mathrm{S} 1-\mathrm{O} 4$ & $1.4428(12)$ & $\mathrm{S} 1-\mathrm{C} 13$ & $1.780(2)$ \\
$\mathrm{S} 1-\mathrm{C} 12$ & $1.781(2)$ & $\mathrm{C} 1-\mathrm{C} 9$ & $1.534(2)$ & $\mathrm{C} 1-\mathrm{C} 12$ & $1.537(2)$ \\
$\mathrm{C} 1-\mathrm{C} 2$ & $1.541(2)$ & $\mathrm{C} 2-\mathrm{C} 3$ & $1.522(2)$ & $\mathrm{C} 3-\mathrm{C} 4$ & $1.525(2)$ \\
$\mathrm{C} 4-\mathrm{C} 5$ & $1.522(3)$ & $\mathrm{C} 5-\mathrm{C} 6$ & $1.511(2)$ & $\mathrm{C} 6-\mathrm{C} 7$ & $1.530(3)$ \\
$\mathrm{C} 6-\mathrm{C} 9$ & $1.571(2)$ & $\mathrm{C} 7-\mathrm{C} 8$ & $1.547(3)$ & $\mathrm{C} 8 \mathrm{C} 11$ & $1.503(2)$ \\
$\mathrm{C} 8-\mathrm{C} 9$ & $1.550(2)$ & $\mathrm{C} 9-\mathrm{O} 1$ & $1.432(2)$ & $\mathrm{C} 10-\mathrm{O} 1$ & $1.409(2)$ \\
$\mathrm{C} 10-\mathrm{O} 2$ & $1.468(2)$ & $\mathrm{C} 10-\mathrm{C} 17$ & $1.502(3)$ & $\mathrm{C} 10-\mathrm{C} 18$ & $1.518(2)$ \\
$\mathrm{C} 11-\mathrm{O} 3$ & $1.197(2)$ & $\mathrm{C} 11-\mathrm{O} 2$ & $1.339(2)$ & $\mathrm{C} 13-\mathrm{C} 14$ & $1.532(2)$ \\
$\mathrm{C} 14-\mathrm{C} 15$ & $1.524(2)$ & $\mathrm{C} 14-\mathrm{C} 16$ & $1.520(2)$ & & \\
\hline
\end{tabular}

Table 5. Bond Angles in Compound 2029,

\begin{tabular}{|llllll|}
\hline O5-S1-O4 & $117.86(7)$ & O5-S1-C13 & $108.51(8)$ & O4-S1-C13 & $108.85(8)$ \\
O5-S1-C12 & $110.16(8)$ & O4-S1-C12 & $106.20(7)$ & C13-S1-C12 & $104.42(7)$ \\
C9-C1-C12 & $107.75(13)$ & C9-C1-C2 & $112.30(13)$ & C12-C1-C2 & $110.74(13)$ \\
C3-C2-C1 & $116.05(14)$ & C4-C3-C2 & $115.50(14)$ & C5-C4-C3 & $113.29(14)$ \\
C6-C5-C4 & $113.8(2)$ & C5-C6-C7 & $116.3(2)$ & C5-C6-C9 & $120.04(14)$ \\
C7-C6-C9 & $89.33(14)$ & C6-C7-C8 & $91.2(2)$ & C11-C8-C7 & $114.7(2)$ \\
C11-C8-C9 & $117.5(2)$ & C7-C8-C9 & $89.49(14)$ & O1-C9-C1 & $103.91(12)$ \\
O1-C9-C8 & $113.83(14)$ & C1-C9-C8 & $115.90(13)$ & O1-C9-C6 & $117.93(14)$ \\
C1-C9-C6 & $116.01(14)$ & C8-C9-C6 & $89.62(13)$ & O1-C10-O2 & $111.07(14)$ \\
O1-C10-C17 & $105.51(14)$ & O2-C10-C17 & $105.6(2)$ & O1-C10-C18 & $114.5(2)$ \\
O2-C10-C18 & $108.0(2)$ & C17-C10-C18 & $111.8(2)$ & O3-C11-O2 & $119.0(2)$ \\
O3-C11-C8 & $122.4(2)$ & O2-C11-C8 & $118.5(2)$ & C1-C12-S1 & $116.83(11)$ \\
C14-C13-S1 & $113.64(12)$ & C15-C14-C16 & $109.7(2)$ & C15-C114-C13 & $108.47(14)$ \\
C16-C14-C13 & $113.01(14)$ & C10-O1-C9 & $120.80(13)$ & C11-O2-C10 & $121.3(2)$ \\
\hline
\end{tabular}




\section{X-ray Structure Determination of Compound 365}
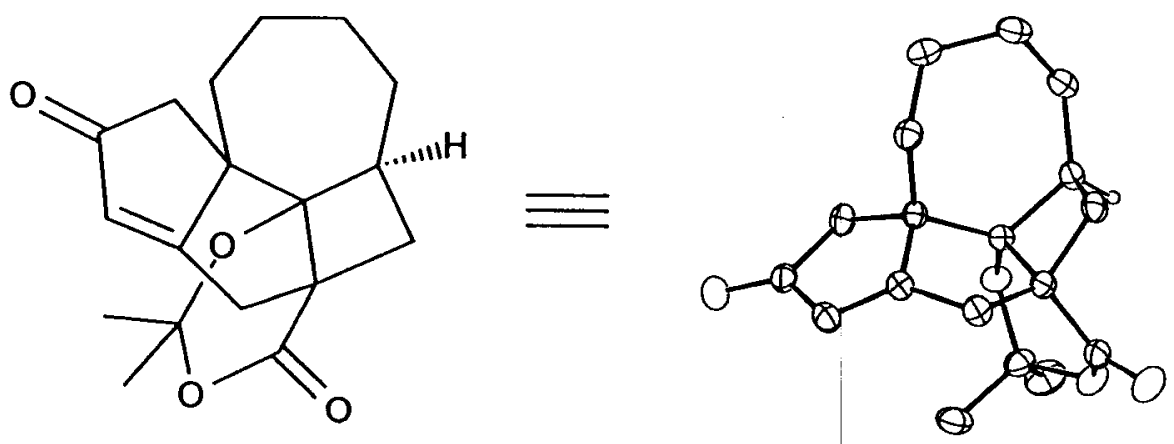

Compound 365, $\mathrm{C}_{18} \mathrm{H}_{22} \mathrm{O}_{4}$, crystallizes in the monoclinic space group $\mathrm{P2} / \mathrm{c}$ (systematic absences OkO: $k=$ odd and hOl: $l=0 d d$ ) with $a=9.876(2) \AA, b=20.661(4) \AA, c=7.631(2) \AA$, $\beta=93.957(5)^{\circ}, V=1553.3(6) \AA^{3}, Z=4$ and $d_{c a l c}=1.293 \mathrm{~g} / \mathrm{cm}^{3}$. X-ray intensity data were collected on a Rigaku Mercury CCD area detector employing graphite-monochromated Mo- $K_{\alpha}$ radiation $(\lambda=0.71069 \AA)$ at a temperature of $295^{\circ} \mathrm{K}$. Indexing was performed from a series of four $0.5^{\circ}$ oscillation images with exposures of 20 seconds. A hemisphere of data was collected using $0.5^{\circ}$ oscillation angles with exposures of 40 seconds per image and a crystal-to-detector distance of $35 \mathrm{~mm}$. Oscillation images were processed using CrystalClear', producing a listing of unaveraged $F^{2}$ and $\sigma\left(F^{2}\right)$ values which were then passed to the teXsan ${ }^{2}$ program package for further processing and structure solution on a Silicon Graphics 02 computer. A total of 9381 reflections were measured over the ranges $5.7 \leq 2 \theta \leq 50.68^{\circ},-11 \leq h \leq 10,-24 \leq k \leq 22,-9 \leq 1$ $\leq 7$ yielding 2824 unique reflections $\left(R_{\mathrm{ind}}=0.0279\right)$. The intensity data were corrected for Lorentz and polarization effects but not for absorption.

The structure was solved by direct methods (SIR92 ${ }^{3}$ ). Refinement was by full-matrix least squares based on $\mathrm{F}^{2}$ using SHELXL-934. All reflections were used during refinement $\left(\mathrm{F}^{2}\right.$ 's that were experimentally negative were replaced by $F^{2}=0$ ). The weighting scheme used was $w=1 /\left[\sigma^{2}\left(F_{0}^{2}\right)+0.0513 P^{2}+0.7167 P\right]$ where $P=\left(F_{0}^{2}+2 F_{c}^{2}\right) / 3$. Non-hydrogen atoms were refined anisotropically and hydrogen atoms were refined using a "riding" model. Refinement converged to $R_{1}=0.0494$ and $w R_{2}=0.1155$ for 2484 reflections for which $F>4 \sigma(F)$ and $R_{1}=0.0569$, 
$w R_{2}=0.1224$ and $G O F=1.081$ for all 2822 unique, non-zero reflections and 201 variables ${ }^{5}$. The maximum $\Delta \sigma$ in the final cycle of least squares was 0.000 and the two most prominent peaks in the final difference Fourier were +0.235 and $-0.238 e / \AA^{3}$.

Table 1. lists cell information, data collection parameters, and refinement data. Final positional and equivalent isotropic thermal parameters are given in Table 2. Anisotropic thermal parameters are in Table 3. Tables 4. and 5. list bond distances and bond angles. Figure 1. is an ORTEP $^{6}$ representation of the molecule with $30 \%$ probability thermal ellipsoids displayed.

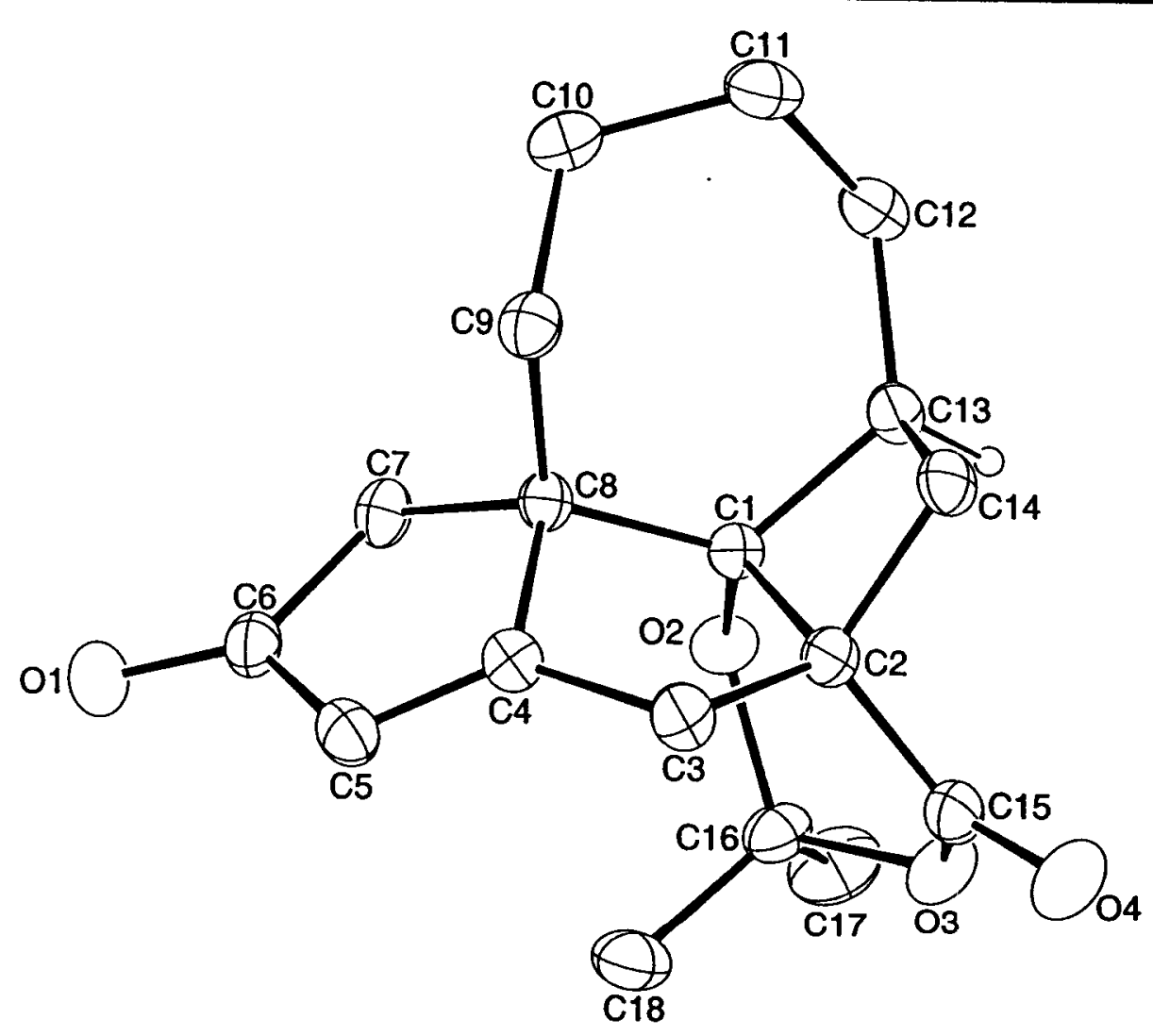

Figure 1. ORTEP drawing of the title compound with $30 \%$ probability thermal ellipsoids. 
Table 1. Summary of Structure Determination of Compound 365

Formula:

Formula weight:

Crystal class:

Space group:

Z

Cell constants:

a

b

c

$\beta$

V

$\mu$

crystal size, $\mathrm{mm}$

$D_{\text {calc }}$

$F(000)$

Radiation:

$2 \theta$ range

hkl collected:

$\leq 7$

No. reflections measured:

No. unique reflections:

No. observed reflections

No. reflections used in refinement

No. parameters

$R$ indices $(F>4 \sigma)$

$R$ indices (all data)

GOF:

Final Difference Peaks, $\mathrm{e} / \mathrm{A}^{3}$
$\mathrm{C}_{18} \mathrm{H}_{22} \mathrm{O}_{4}$

302.36

monoclinic

$\mathrm{P} 2$ /c (\#14)

4

$9.876(2) \AA$

20.661(4)A

$7.631(2) \AA$

$93.957(5)^{\circ}$

1553.3(6) $\AA^{3}$

$0.90 \mathrm{~cm}^{-1}$

$0.46 \times 0.14 \times 0.05$

$1.293 \mathrm{~g} / \mathrm{cm}^{3}$

648

Mo-K $K_{\alpha}(\lambda=0.71069 \AA)$

$5.7-50.68^{\circ}$

$-11 \leq h \leq 10 ;-24 \leq k \leq 22 ;-9 \leq 1$

9381

$2824\left(R_{\text {int }}=0.0279\right)$

2484 ( $F>4 \sigma)$

2822

201

$R_{1}=0.0494$

$w R_{2}=0.1155$

$R_{1}=0.0569$

$w R_{2}=0.1224$

1.081

$+0.235,-0.238$ 
Table 2. Refined Positional Parameters for Compound 365

\begin{tabular}{|c|c|c|c|c|}
\hline Atom & $x$ & $y$ & $\mathbf{z}$ & $U_{e q}, A^{2}$ \\
\hline $\mathrm{Cl}$ & $0.2560(2)$ & $0.35942(8)$ & $0.6338(2)$ & $0.0279(4)$ \\
\hline $\mathrm{C} 2$ & $0.3221(2)$ & $0.39393(8)$ & $0.4793(2)$ & $0.0319(4)$ \\
\hline C3 & $0.2920(2)$ & $0.46766(9)$ & $0.4871(2)$ & $0.0375(4)$ \\
\hline H3a & $0.3750(2)$ & $0.49290(9)$ & $0.4908(2)$ & 0.050 \\
\hline $\mathrm{H} 3 \mathrm{~b}$ & $0.2326(2)$ & $0.48142(9)$ & $0.3875(2)$ & 0.050 \\
\hline $\mathrm{C} 4$ & $0.2232(2)$ & $0.47325(8)$ & $0.6558(2)$ & $0.0333(4)$ \\
\hline C5 & $0.2243(2)$ & $0.51662(9)$ & $0.7848(2)$ & $0.0408(4)$ \\
\hline H5 & $0.2551(2)$ & $0.55903(9)$ & $0.7784(2)$ & 0.054 \\
\hline C6 & $0.1690(2)$ & $0.48672(10)$ & $0.9385(2)$ & $0.0389(4)$ \\
\hline $\mathrm{C7}$ & $0.1384(2)$ & $0.41603(9)$ & $0.8967(2)$ & $0.0365(4)$ \\
\hline $\mathrm{H} 7 \mathrm{a}$ & $0.0471(2)$ & $0.40488(9)$ & $0.9252(2)$ & 0.049 \\
\hline $\mathrm{H} 7 \mathrm{~b}$ & $0.2022(2)$ & $0.38786(9)$ & $0.9620(2)$ & 0.049 \\
\hline C8 & $0.1533(2)$ & $0.41015(8)$ & $0.6982(2)$ & $0.0301(4)$ \\
\hline $\mathrm{C} 9$ & $0.0114(2)$ & $0.41249(9)$ & $0.5981(2)$ & $0.0377(4)$ \\
\hline $\mathrm{H} 9 \mathrm{a}$ & $-0.0365(2)$ & $0.44970(9)$ & $0.6405(2)$ & 0.050 \\
\hline $\mathrm{H} 9 \mathrm{~b}$ & $0.0245(2)$ & $0.42013(9)$ & $0.4750(2)$ & 0.050 \\
\hline C10 & $-0.0806(2)$ & $0.35368(10)$ & $0.6097(3)$ & $0.0466(5)$ \\
\hline $\mathrm{H} 10 \mathrm{a}$ & $-0.0698(2)$ & $0.33641(10)$ & $0.7280(3)$ & 0.062 \\
\hline H10b & $-0.1742(2)$ & $0.36764(10)$ & $0.5893(3)$ & 0.062 \\
\hline C11 & $-0.0540(2)$ & $0.29970(11)$ & $0.4808(3)$ & $0.0488(5)$ \\
\hline H11a & $-0.1268(2)$ & $0.26821(11)$ & $0.4827(3)$ & 0.065 \\
\hline $\mathrm{H} 11 \mathrm{~b}$ & $-0.0562(2)$ & $0.31779(11)$ & $0.3633(3)$ & 0.065 \\
\hline $\mathrm{C} 12$ & $0.0804(2)$ & $0.26520(9)$ & $0.5188(2)$ & $0.0407(4)$ \\
\hline
\end{tabular}




\begin{tabular}{|c|c|c|c|c|}
\hline $\mathrm{H} 12 \mathrm{a}$ & $0.0832(2)$ & $0.22884(9)$ & $0.4387(2)$ & 0.054 \\
\hline $\mathrm{H} 12 \mathrm{~b}$ & $0.0813(2)$ & $0.24757(9)$ & $0.6367(2)$ & 0.054 \\
\hline C13 & $0.2102(2)$ & $0.30413(8)$ & $0.5055(2)$ & $0.0334(4)$ \\
\hline $\mathrm{H} 13$ & $0.2845(2)$ & $0.27265(8)$ & $0.5105(2)$ & 0.044 \\
\hline C14 & $0.2347(2)$ & $0.35001(9)$ & $0.3496(2)$ & $0.0385(4)$ \\
\hline $\mathrm{H} 14 \mathrm{a}$ & $0.1529(2)$ & $0.37075(9)$ & $0.2991(2)$ & 0.051 \\
\hline $\mathrm{H} 14 \mathrm{~b}$ & $0.2855(2)$ & $0.33033(9)$ & $0.2592(2)$ & 0.051 \\
\hline C15 & $0.4698(2)$ & $0.37858(9)$ & $0.4662(2)$ & $0.0392(4)$ \\
\hline C16 & $0.4853(2)$ & $0.35287(10)$ & $0.7795(2)$ & $0.0392(4)$ \\
\hline C17 & $0.5599(2)$ & $0.30024(12)$ & $0.8831(3)$ & $0.0609(6)$ \\
\hline $\mathrm{H} 17 \mathrm{a}$ & $0.6558(2)$ & $0.3081(5)$ & $0.885(2)$ & 0.091 \\
\hline $\mathrm{H17b}$ & $0.5396(14)$ & $0.2591(2)$ & $0.8290(13)$ & 0.091 \\
\hline H17c & $0.5318(13)$ & $0.3000(6)$ & $1.0011(7)$ & 0.091 \\
\hline C18 & $0.5167(2)$ & $0.41936(11)$ & $0.8534(3)$ & $0.0515(5)$ \\
\hline $\mathrm{H} 18 \mathrm{a}$ & $0.6128(3)$ & $0.4267(3)$ & $0.857(2)$ & 0.077 \\
\hline$H 18 b$ & $0.4864(14)$ & $0.4222(3)$ & $0.9701(8)$ & 0.077 \\
\hline $\mathrm{H} 18 \mathrm{c}$ & $0.4709(13)$ & $0.45141(12)$ & $0.7801(11)$ & 0.077 \\
\hline 01 & $0.15673(14)$ & $0.51212(8)$ & $1.0809(2)$ & $0.0532(4)$ \\
\hline $\mathrm{O} 2$ & $0.34585(11)$ & $0.33654(6)$ & $0.7756(2)$ & $0.0342(3)$ \\
\hline 03 & $0.53609(13)$ & $0.34940(7)$ & $0.6041(2)$ & $0.0486(4)$ \\
\hline 04 & $0.5288(2)$ & $0.38793(8)$ & $0.3359(2)$ & $0.0594(4)$ \\
\hline
\end{tabular}


Table 3. Refined Thermal Parameters (U's) for Compound 365

\begin{tabular}{|c|c|c|c|c|c|c|}
\hline & $U_{11}$ & $U_{22}$ & $\mathrm{U}_{33}$ & $\mathrm{U}_{23}$ & $U_{13}$ & $U_{12}$ \\
\hline C1 & $0.0295(8)$ & $0.0297(9)$ & $0.0243(8)$ & $0.0027(6)$ & $0.0009(6)$ & $-0.0005(6)$ \\
\hline $\mathrm{C} 2$ & $0.0357(9)$ & $0.0349(10)$ & $0.0256(8)$ & $0.0015(7)$ & $0.0054(7)$ & $-0.0032(7)$ \\
\hline C3 & $0.0458(10)$ & $0.0344(10)$ & $0.0327(9)$ & $0.0045(8)$ & $0.0055(7)$ & $-0.0030(8)$ \\
\hline $\mathrm{C} 4$ & $0.0362(9)$ & $0.0312(9)$ & $0.0320(9)$ & $0.0028(7)$ & $-0.0004(7)$ & $0.0020(7)$ \\
\hline C5 & $0.0450(11)$ & $0.0348(10)$ & $0.0425(10)$ & $-0.0051(8)$ & $0.0018(8)$ & $-0.0007(8)$ \\
\hline C6 & $0.0317(9)$ & $0.0517(12)$ & $0.0329(9)$ & $-0.0093(8)$ & $0.0005(7)$ & $0.0055(8)$ \\
\hline C7 & $0.0407(10)$ & $0.0413(10)$ & $0.0283(9)$ & $0.0005(7)$ & $0.0073(7)$ & $0.0055(8)$ \\
\hline $\mathrm{C} 8$ & $0.0327(9)$ & $0.0311(9)$ & $0.0266(8)$ & $0.0008(7)$ & $0.0027(7)$ & $0.0025(7)$ \\
\hline C9 & $0.0352(10)$ & $0.0403(10)$ & $0.0374(10)$ & $0.0019(8)$ & $0.0005(7)$ & $0.0061(8)$ \\
\hline C10 & $0.0306(10)$ & $0.0547(13)$ & $0.0541(12)$ & $0.0013(10)$ & $0.0001(8)$ & $-0.0008(8)$ \\
\hline C11 & $0.0409(11)$ & $0.0529(13)$ & $0.0514(12)$ & $-0.0042(10)$ & $-0.0051(9)$ & $-0.0113(9)$ \\
\hline C12 & $0.0465(11)$ & $0.0381(10)$ & $0.0370(10)$ & $-0.0021(8)$ & $-0.0007(8)$ & $-0.0096(8)$ \\
\hline C13 & $0.0380(9)$ & $0.0309(9)$ & $0.0315(9)$ & $-0.0029(7)$ & $0.0035(7)$ & $-0.0006(7)$ \\
\hline C14 & $0.0450(10)$ & $0.0447(11)$ & $0.0261(9)$ & $-0.0043(8)$ & $0.0052(7)$ & $-0.0041(8)$ \\
\hline C15 & $0.0424(10)$ & $0.0385(10)$ & $0.0377(10)$ & $-0.0046(8)$ & $0.0103(8)$ & $-0.0054(8)$ \\
\hline C16 & $0.0281(9)$ & $0.0528(12)$ & $0.0364(10)$ & $0.0030(8)$ & $0.0002(7)$ & $0.0002(8)$ \\
\hline C17 & $0.0414(12)$ & $0.073(2)$ & $0.067(2)$ & $0.0180(12)$ & $-0.0068(10)$ & $0.0115(11)$ \\
\hline C18 & $0.0439(12)$ & $0.0596(14)$ & $0.0497(12)$ & $-0.0042(10)$ & $-0.0056(9)$ & $-0.0133(10)$ \\
\hline 01 & $0.0475(8)$ & $0.0717(10)$ & $0.0406(8)$ & $-0.0225(7)$ & $0.0042(6)$ & $0.0003(7)$ \\
\hline $\mathrm{O} 2$ & $0.0297(6)$ & $0.0412(7)$ & $0.0311(6)$ & $0.0067(5)$ & $-0.0020(5)$ & $-0.0009(5)$ \\
\hline O3 & $0.0328(7)$ & $0.0685(10)$ & $0.0452(8)$ & $0.0013(7)$ & $0.0077(6)$ & $0.0058(6)$ \\
\hline 04 & $0.0544(9)$ & $0.0754(11)$ & $0.0518(9)$ & $0.0043(8)$ & $0.0275(7)$ & $-0.0045(8)$ \\
\hline
\end{tabular}

The form of the anisotropic displacement parameter is:

$\exp \left[-2 \pi^{2}\left(a^{\star 2} U_{11} h^{2}+b^{\star 2} U_{22} k^{2}+c^{\star 2} U_{33}{ }^{2}+2 b^{*} c^{*} U_{23} k l+2 a^{*} c^{\star} U_{13} h l+2 a^{*} b^{*} U_{12} h k\right)\right]$. 
Table 4. Bond Distances in Compound 365, A

\begin{tabular}{|llllll|}
\hline $\mathrm{C} 1-\mathrm{O} 2$ & $1.432(2)$ & $\mathrm{C} 1-\mathrm{C} 13$ & $1.551(2)$ & $\mathrm{C} 1-\mathrm{C} 8$ & $1.561(2)$ \\
$\mathrm{C} 1-\mathrm{C} 2$ & $1.560(2)$ & $\mathrm{C} 2-\mathrm{C} 15$ & $1.502(3)$ & $\mathrm{C} 2-\mathrm{C} 3$ & $1.554(3)$ \\
$\mathrm{C} 2-\mathrm{C} 14$ & $1.559(2)$ & $\mathrm{C} 3-\mathrm{C} 4$ & $1.501(2)$ & $\mathrm{C} 4-\mathrm{C} 5$ & $1.331(3)$ \\
$\mathrm{C} 4-\mathrm{C} 8$ & $1.521(2)$ & $\mathrm{C} 5-\mathrm{C} 6$ & $1.464(3)$ & $\mathrm{C} 6-\mathrm{O} 1$ & $1.221(2)$ \\
$\mathrm{C} 6-\mathrm{C} 7$ & $1.521(3)$ & $\mathrm{C} 7-\mathrm{C} 8$ & $1.537(2)$ & $\mathrm{C} 8-\mathrm{C} 9$ & $1.549(2)$ \\
$\mathrm{C} 9-\mathrm{C} 10$ & $1.524(3)$ & $\mathrm{C} 10-\mathrm{C} 11$ & $1.522(3)$ & $\mathrm{C} 11-\mathrm{C} 12$ & $1.517(3)$ \\
$\mathrm{C} 12-\mathrm{C} 13$ & $1.523(2)$ & $\mathrm{C} 13-\mathrm{C} 14$ & $1.553(2)$ & $\mathrm{C} 15-\mathrm{O} 4$ & $1.203(2)$ \\
$\mathrm{C} 15-\mathrm{O} 3$ & $1.343(2)$ & $\mathrm{C} 16-\mathrm{O} 2$ & $1.416(2)$ & $\mathrm{C} 16-\mathrm{O} 3$ & $1.463(2)$ \\
$\mathrm{C} 16-\mathrm{C} 17$ & $1.507(3)$ & $\mathrm{C} 16-\mathrm{C} 18$ & $1.509(3)$ & & \\
\hline
\end{tabular}


Table 5. Bond Angles in Compound 365 , $^{\circ}$

\begin{tabular}{|c|c|c|c|c|c|}
\hline $\mathrm{O} 2-\mathrm{C} 1-\mathrm{C} 13$ & $111.86(13)$ & $\mathrm{O} 2-\mathrm{C} 1-\mathrm{C} 8$ & $111.49(13)$ & C13-C1-C8 & $121.71(13)$ \\
\hline $\mathrm{O} 2-\mathrm{C} 1-\mathrm{C} 2$ & $116.87(13)$ & C13-C1-C2 & $88.78(12)$ & $\mathrm{C} 8-\mathrm{C} 1-\mathrm{C} 2$ & $104.25(13)$ \\
\hline $\mathrm{C} 15-\mathrm{C} 2-\mathrm{C} 3$ & $113.5(2)$ & C15-C2-C14 & $109.4(2)$ & C3-C2-C14 & $119.7(2)$ \\
\hline C15-C2-C1 & $114.39(14)$ & C3-C2-C1 & $109.14(13)$ & $\mathrm{C} 14-\mathrm{C} 2-\mathrm{C} 1$ & $88.33(13)$ \\
\hline $\mathrm{C} 4-\mathrm{C} 3-\mathrm{C} 2$ & $101.97(13)$ & $\mathrm{C} 5-\mathrm{C} 4-\mathrm{C} 3$ & $134.9(2)$ & C5-C4-C8 & $113.6(2)$ \\
\hline C3-C4-C8 & $110.82(14)$ & C4-C5-C6 & $108.9(2)$ & $\mathrm{O} 1-\mathrm{C} 6-\mathrm{C} 5$ & $126.7(2)$ \\
\hline $\mathrm{O} 1-\mathrm{C} 6-\mathrm{C} 7$ & $124.7(2)$ & $\mathrm{C} 5-\mathrm{C} 6-\mathrm{C} 7$ & $108.5(2)$ & $\mathrm{C} 6-\mathrm{C} 7-\mathrm{C} 8$ & $104.56(14)$ \\
\hline $\mathrm{C} 4-\mathrm{C} 8-\mathrm{C} 7$ & $102.57(14)$ & C4-C8-C9 & $106.08(14)$ & C7-C8-C9 & $109.77(14)$ \\
\hline $\mathrm{C} 4-\mathrm{C} 8-\mathrm{C} 1$ & $101.22(13)$ & $\mathrm{C} 7-\mathrm{C} 8-\mathrm{C} 1$ & $118.15(13)$ & $\mathrm{C9}-\mathrm{C} 8-\mathrm{C} 1$ & $116.80(14)$ \\
\hline C10-C9-C8 & $118.0(2)$ & C11-C10-C9 & $114.6(2)$ & C12-C11-C10 & $114.1(2)$ \\
\hline $\mathrm{C} 11-\mathrm{C} 12-\mathrm{C} 13$ & $117.9(2)$ & $\mathrm{C} 12-\mathrm{C} 13-\mathrm{C} 1$ & $123.8(2)$ & $\mathrm{C}_{12}-\mathrm{C}_{13}-\mathrm{C}_{14}$ & $123.4(2)$ \\
\hline $\mathrm{C} 1-\mathrm{C} 13-\mathrm{C} 14$ & $88.84(13)$ & C13-C14-C2 & $88.75(12)$ & O4-C15-O3 & $118.8(2)$ \\
\hline O4-C15-C2 & $123.3(2)$ & $\mathrm{O} 3-\mathrm{C} 15-\mathrm{C} 2$ & $117.8(2)$ & $\mathrm{O} 2-\mathrm{C} 16-\mathrm{O} 3$ & $111.30(14)$ \\
\hline $\mathrm{O} 2-\mathrm{C} 16-\mathrm{C} 17$ & $106.2(2)$ & O3-C16-C17 & $105.0(2)$ & $\mathrm{O} 2-\mathrm{C} 16-\mathrm{C} 18$ & $113.5(2)$ \\
\hline O3-C16-C18 & $108.2(2)$ & C17-C16-C18 & $112.3(2)$ & $\mathrm{C} 16-\mathrm{O} 2-\mathrm{C} 1$ & $119.25(13)$ \\
\hline C15-O3-C16 & $121.00(14)$ & & & & \\
\hline
\end{tabular}




\section{References}

1. CrystalClear: Rigaku Corporation, 1999.

2. teXsan: Crystal Structure Analysis Package, Molecular Structure Corporation (1985 \& 1992).

3. SIR92: Altomare, A., Burla, M.C., Camalli, M., Cascarano, M., Giacovazzo, C., Guagliardi, A., Polidoro, G. (1994). J. Appl. Cryst., 27, 435.

4. SHELXL-93: Program for the Refinement of Crystal Structures, Sheldrick, G.M. (1993), University of Göttingen, Germany.

5. $R_{1}=\sum|| F_{0}|-| F_{c}\left|/ \sum\right| F_{0} \mid$

$w R_{2}=\left\{\sum w\left(F_{0}^{2}-F_{c}^{2}\right)^{2} / \sum w\left(F_{0}^{2}\right)^{2}\right\}^{1 / 2}$

GOF $=\left\{\sum w\left(F_{0}^{2}-F_{c}^{2}\right)^{2} /(n-p)\right\}^{1 / 2}$

where $n=$ the number of reflections and $p=$ the number of parameters refined.

6. "ORTEP-II: A Fortran Thermal Ellipsoid Plot Program for Crystal Structure Illustrations". C.K. Johnson (1976) ORNL-5138. 


\section{X-ray Structure Determination of Compound 2033}

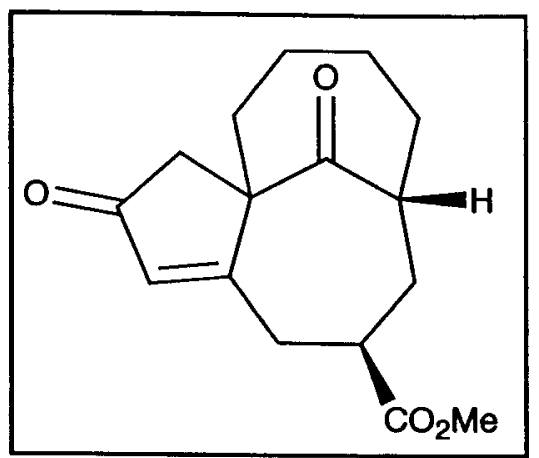

Compound 2033, $\mathrm{C}_{16} \mathrm{H}_{20} \mathrm{O}_{4}$, crystallizes in the monoclinic space group $\mathrm{C} 2 / \mathrm{c}$ (systematic absences $h k l: h+k=0 d d$ and hol: l=odd) with $a=29.841(3) \AA \AA, b=8.4289(5) \AA, c=23.601(2) \AA, \quad \beta=107.4780(10)^{\circ}$, $V=5662.1(8) \AA^{3}, Z=16$ and $d_{\text {calc }}=1.297 \mathrm{~g} / \mathrm{cm}^{3}$. X-ray intensity data were collected on a Rigaku Mercury CCD area detector employing graphite-monochromated $\mathrm{Mo-} \mathrm{K}_{\alpha}$ radiation $(\lambda=0.71069 \AA)$ at a temperature of $143 \mathrm{~K}$. Preliminary indexing was performed from a series of twelve $0.5^{\circ}$ rotation images with exposures of 30 seconds. A total of 348 rotation images were collected with a crystal to detector distance of $36 \mathrm{~mm}$, a $2 \theta$ swing angle of $-10^{\circ}$, rotation widths of $0.5^{\circ}$ and exposures of 60 seconds: scan no. 1 was a $\phi$-scan from $150^{\circ}$ to $324^{\circ}$ at $\omega=0^{\circ}$ and $\chi=-30^{\circ}$. Rotation images were processed using CrystalClear ${ }^{1}$, producing a listing of unaveraged $F^{2}$ and $\sigma\left(F^{2}\right)$ values which were then passed to the Crystalstructure ${ }^{2}$ program package for further processing and structure solution on a Dell Pentium III computer. A total of 12343 reflections were measured over the ranges $5.04 \leq 2 \theta \leq 50.7^{\circ},-35 \leq h \leq 35,-9 \leq k \leq 10,-25 \leq 1$ $\leq 28$ yielding 5092 unique reflections $\left(R_{\text {int }}=0.0161\right)$. The intensity data were corrected for Lorentz and polarization effects and for absorption. using REQAB ${ }^{3}$ (minimum and maximum transmission 0.859, 1.000)

The structure was solved by direct methods $\left(\operatorname{SIR} 97^{4}\right)$. Refinement was by full-matrix least squares based on $F^{2}$ using SHELXL-97 $7^{5}$. All reflections were used during refinement $\left(F^{2}\right.$ 's that were experimentally negative were replaced by $\left.F^{2}=0\right)$. The weighting scheme used was $w=1 /\left[\sigma^{2}\left(F_{0}^{2}\right)+\right.$ $\left.0.0574 \mathrm{P}^{2}+5.4789 \mathrm{P}\right]$ where $\mathrm{P}=\left(\mathrm{F}_{\mathrm{o}}^{2}+2 \mathrm{~F}_{\mathrm{c}}^{2}\right) / 3$. Non-hydrogen atoms were refined anisotropically and hydrogen atoms were refined using a "riding" model. Refinement converged to $R_{1}=0.0461$ and $w R_{2}=0.1145$ for 4503 reflections for which $F>4 \sigma(F)$ and $R_{1}=0.0524, w R_{2}=0.1200$ and $G O F=1.040$ for all 
5092 unique, non-zero reflections and 364 variables $^{6}$. The maximum $\Delta / \sigma$ in the final cycle of least squares was 0.001 and the two most prominent peaks in the final difference Fourier were +0.582 and $0.327 \mathrm{e} / \AA^{3}$.

Table 1. lists cell information, data collection parameters, and refinement data. Final positional and equivalent isotropic thermal parameters are given in Table 2. Anisotropic thermal parameters are in Table 3. Tables 4. and 5. list bond distances and bond angles. Figures 1. and 2. are ORTEP ${ }^{7}$ representation of the molecule with $30 \%$ probability thermal ellipsoids displayed.

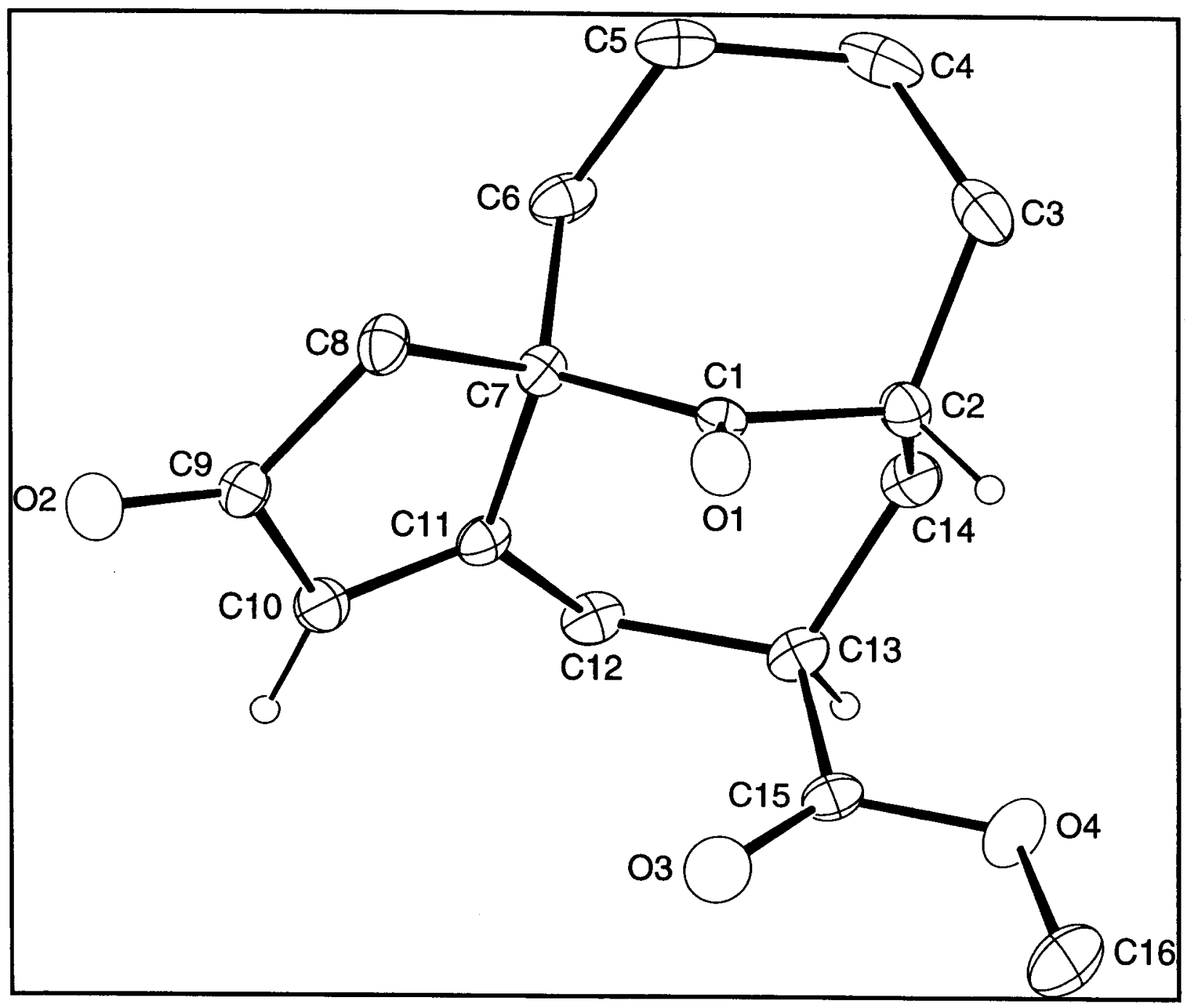

Figure 1. ORTEP drawing of molecule no. 1 of the asymmetric unit with $30 \%$ probability thermal ellipsoids. 


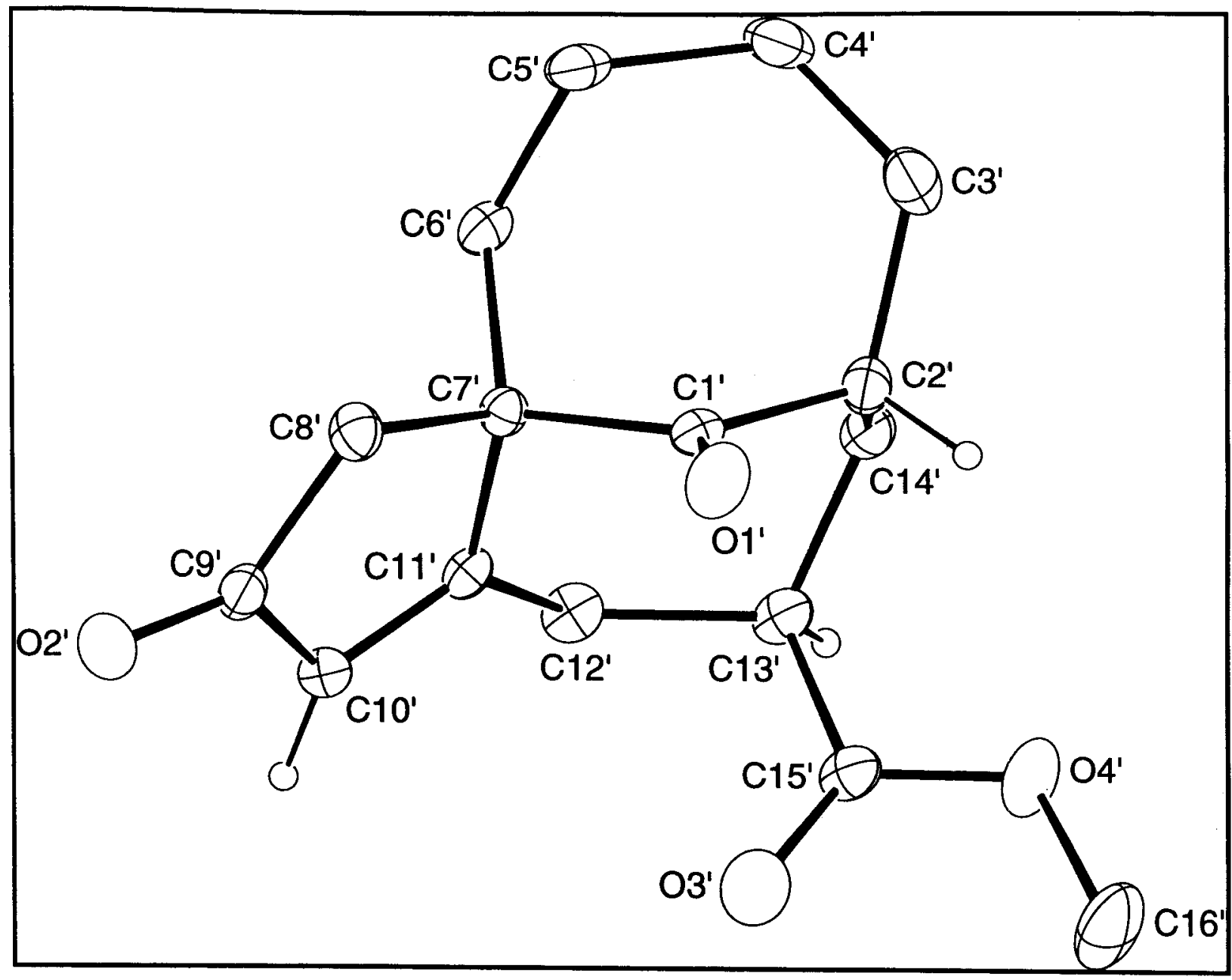

Figure 2. ORTEP drawing of molecule no. 2 of the asymmetric unit with $30 \%$ probability thermal ellipsoids.

\section{References}

1. CrystalClear: Rigaku Corporation, 1999.

2. CrystalStructure: Crystal Structure Analysis Package, Rigaku Corp. Rigaku/MSC (2002).

3. REQAB4: R.A. Jacobsen, (1994). Private Communication.

4. SIR97: Altomare, A., M. Burla, M. Camalli, G. Cascarano, C. Giacovazzo, A. Guagliardi, A. Moliterni, G. Polidori \& R. Spagna (1999). J. Appl. Cryst., 32, 115-119.

5. SHELXL-97: Program for the Refinement of Crystal Structures, Sheldrick, G.M. (1997), University of Göttingen, Germany. 
6. $R_{1}=\sum\left\|F_{0}|-| F_{c}\right\| / \sum I F_{0} \mid$

$w R_{2}=\left\{\sum w\left(F_{0}^{2}-F_{c}^{2}\right)^{2} / \sum w\left(F_{0}^{2}\right)^{2}\right\}^{1 / 2}$

GOF $=\left\{\sum w\left(F_{0}^{2}-F_{c}^{2}\right)^{2} /(n-p)\right\}^{1 / 2}$

where $n=$ the number of reflections and $p=$ the number of parameters refined.

7. "ORTEP-II: A Fortran Thermal Ellipsoid Plot Program for Crystal Structure Illustrations". C.K. Johnson (1976) ORNL-5138. 
Table 1. Summary of Structure Determination of Compound 2033

Formula:

Formula weight:

Crystal class:

Space group:

Z

Cell constants:

a

b

C

$\beta$

V

$\mu$

crystal size, mm

$D_{\text {calc }}$

$\mathrm{F}(000)$

Radiation:

$2 \theta$ range

hkl collected:

No. reflections measured:

No. unique reflections:

No. observed reflections

No. reflections used in refinement

No. parameters

$R$ indices $(F>4 \sigma)$

$R$ indices (all data)

GOF:

Final Difference Peaks, e/ $\AA^{3}$
$\mathrm{C}_{16} \mathrm{H}_{20} \mathrm{O}_{4}$

276.32

monoclinic

$\mathrm{C} 2 / \mathrm{c}(\# 15)$

16

$29.841(3) \AA$

$8.4289(5) \AA$

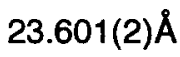

$107.4780(10)^{\circ}$

$5662.1(8) \AA^{3}$

$0.92 \mathrm{~cm}^{-1}$

$0.30 \times 0.30 \times 0.28$

$1.297 \mathrm{~g} / \mathrm{cm}^{3}$

2368

Mo-K $K_{\alpha}(\lambda=0.71069 \AA)$

$5.04-50.7^{\circ}$

$-35 \leq h \leq 35 ;-9 \leq k \leq 10 ;-25 \leq 1 \leq 28$

12343

$5092\left(\mathrm{R}_{\mathrm{int}}=0.0161\right)$

4503 (F>4o)

5092

364

$R_{1}=0.0461$

$w R_{2}=0.1145$

$R_{1}=0.0524$

$w^{2} R_{2}=0.1200$

1.040

$+0.582,-0.327$ 
Table 2. Refined Positional Parameters for Compound 2033

\begin{tabular}{|c|c|c|c|c|}
\hline Atom & $x$ & $y$ & $z$ & $U_{\text {eq }}, \AA^{2}$ \\
\hline C1 & $0.36121(5)$ & $0.5144(2)$ & $0.24502(6)$ & $0.0200(3)$ \\
\hline $\mathrm{C} 2$ & $0.32404(5)$ & $0.5175(2)$ & $0.18474(7)$ & $0.0264(3)$ \\
\hline $\mathrm{H} 2$ & 0.2956 & 0.5594 & 0.1920 & 0.035 \\
\hline C3 & $0.31226(6)$ & $0.3443(2)$ & $0.16390(8)$ & $0.0392(4)$ \\
\hline $\mathrm{H} 3 \mathrm{a}$ & 0.3049 & 0.2864 & 0.1955 & 0.052 \\
\hline $\mathrm{H} 3 \mathrm{~b}$ & 0.2841 & 0.3453 & 0.1300 & 0.052 \\
\hline C4 & $0.35024(7)$ & $0.2537(2)$ & $0.14660(8)$ & $0.0427(5)$ \\
\hline $\mathrm{H} 4 \mathrm{a}$ & 0.3543 & 0.3022 & 0.1112 & 0.057 \\
\hline $\mathrm{H} 4 \mathrm{~b}$ & 0.3393 & 0.1460 & 0.1364 & 0.057 \\
\hline C5 & $0.39803(7)$ & $0.2468(2)$ & $0.19383(8)$ & $0.0380(4)$ \\
\hline $\mathrm{H} 5 \mathrm{a}$ & 0.4171 & 0.1670 & 0.1824 & 0.051 \\
\hline $\mathrm{H} 5 \mathrm{~b}$ & 0.3936 & 0.2136 & 0.2311 & 0.051 \\
\hline C6 & $0.42471(6)$ & $0.4049(2)$ & $0.20349(8)$ & $0.0311(4)$ \\
\hline H6a & 0.4171 & 0.4613 & 0.1659 & 0.041 \\
\hline $\mathrm{H} 6 \mathrm{~b}$ & 0.4582 & 0.3830 & 0.2156 & 0.041 \\
\hline $\mathrm{C7}$ & $0.41386(5)$ & $0.5139(2)$ & $0.25032(7)$ & $0.0224(3)$ \\
\hline C8 & $0.44355(5)$ & $0.4698(2)$ & $0.31393(7)$ & $0.0288(4)$ \\
\hline $\mathrm{H} 8 \mathrm{a}$ & 0.4234 & 0.4470 & 0.3384 & 0.038 \\
\hline $\mathrm{H} 8 \mathrm{~b}$ & 0.4627 & 0.3772 & 0.3134 & 0.038 \\
\hline $\mathrm{C} 9$ & $0.47412(5)$ & $0.6119(2)$ & $0.33758(7)$ & $0.0276(4)$ \\
\hline C10 & $0.46081(5)$ & $0.7371(2)$ & $0.29324(7)$ & $0.0279(4)$ \\
\hline $\mathrm{H} 10$ & 0.4734 & 0.8389 & 0.2982 & 0.037 \\
\hline $\mathrm{C} 11$ & $0.42816(5)$ & $0.6867(2)$ & $0.24443(7)$ & $0.0235(3)$ \\
\hline $\mathrm{C} 12$ & $0.40945(5)$ & $0.7810(2)$ & $0.18863(7)$ & $0.0272(4)$ \\
\hline $\mathrm{H} 12 \mathrm{a}$ & 0.4233 & 0.7392 & 0.1595 & 0.036 \\
\hline $\mathrm{H} 12 \mathrm{~b}$ & 0.4205 & 0.8892 & 0.1970 & 0.036 \\
\hline $\mathrm{C} 13$ & $0.35588(5)$ & $0.7865(2)$ & $0.15977(7)$ & $0.0263(3)$ \\
\hline $\mathrm{H} 13$ & 0.3504 & 0.8548 & 0.1247 & 0.035 \\
\hline $\mathrm{C} 14$ & $0.33340(6)$ & $0.6254(2)$ & $0.13715(7)$ & $0.0299(4)$ \\
\hline $\mathrm{H} 14 \mathrm{a}$ & 0.3038 & 0.6444 & 0.1066 & 0.040 \\
\hline $\mathrm{H} 14 \mathrm{~b}$ & 0.3538 & 0.5696 & 0.1187 & 0.040 \\
\hline C15 & $0.33128(5)$ & $0.8654(2)$ & $0.20004(7)$ & $0.0262(3)$ \\
\hline$C_{16}$ & $0.25715(7)$ & $0.9413(3)$ & $0.20793(9)$ & $0.0464(5)$ \\
\hline $\mathrm{H} 16 \mathrm{a}$ & 0.2690 & 1.0439 & 0.2227 & 0.070 \\
\hline $\mathrm{H} 16 \mathrm{~b}$ & 0.2588 & 0.8717 & 0.2407 & 0.070 \\
\hline $\mathrm{H} 16 \mathrm{c}$ & 0.2251 & 0.9510 & 0.1837 & 0.070 \\
\hline 01 & $0.34832(4)$ & $0.51041(14)$ & $0.28910(5)$ & $0.0300(3)$ \\
\hline $\mathrm{O} 2$ & $0.50428(4)$ & $0.6182(2)$ & $0.38585(5)$ & $0.0403(3)$ \\
\hline $\mathrm{O} 3$ & $0.34975(4)$ & $0.9116(2)$ & $0.24954(5)$ & $0.0418(3)$ \\
\hline O4 & $0.28503(4)$ & $0.8770(2)$ & $0.17277(5)$ & $0.0402(3)$ \\
\hline$C 1^{\prime}$ & $0.63772(5)$ & $0.0401(2)$ & $0.51155(7)$ & $0.0244(3)$ \\
\hline $\mathrm{C} 2^{\prime}$ & $0.67005(5)$ & $0.0743(2)$ & $0.57379(7)$ & $0.0311(4)$ \\
\hline $\mathrm{H} 2^{\prime}$ & 0.7000 & 0.1078 & 0.5686 & 0.041 \\
\hline $\mathrm{C}^{\prime}$ & $0.68026(6)$ & $-0.0842(2)$ & $0.60803(9)$ & $0.0437(5)$ \\
\hline H3'1 & 0.6918 & -0.1591 & 0.5845 & 0.058 \\
\hline H3'2 & 0.7054 & -0.0665 & 0.6446 & 0.058 \\
\hline C4' & $0.63919(7)$ & $-0.1611(2)$ & $0.62372(8)$ & $0.0428(5)$ \\
\hline $\mathrm{H} 44^{\prime} 1$ & 0.6310 & -0.0947 & 0.6527 & 0.057 \\
\hline
\end{tabular}




\begin{tabular}{|c|c|c|c|c|}
\hline $\mathrm{H}^{\prime \prime 2}$ & 0.6495 & -0.2626 & 0.6425 & 0.057 \\
\hline C5' & $0.59492(6)$ & $-0.1882(2)$ & $0.57138(8)$ & $0.0363(4)$ \\
\hline H5'1 & 0.6037 & -0.2403 & 0.5396 & 0.048 \\
\hline $\mathrm{H} 5{ }^{\prime} 2$ & 0.5741 & -0.2589 & 0.5839 & 0.048 \\
\hline C6' & $0.56835(5)$ & $-0.0355(2)$ & $0.54719(7)$ & $0.0290(4)$ \\
\hline H6'1 & 0.5722 & 0.0383 & 0.5799 & 0.039 \\
\hline H6'2 & 0.5351 & -0.0600 & 0.5317 & 0.039 \\
\hline $\mathrm{C} 7^{\prime}$ & $0.58399(5)$ & $0.0478(2)$ & $0.49776(6)$ & $0.0228(3)$ \\
\hline $\mathrm{C}^{\prime}$ & $0.55940(6)$ & $-0.0220(2)$ & $0.43593(7)$ & $0.0297(4)$ \\
\hline H8'1 & 0.5824 & -0.0593 & 0.4174 & 0.039 \\
\hline H8'2 & 0.5395 & -0.1104 & 0.4391 & 0.039 \\
\hline $\mathrm{CP}^{\prime}$ & $0.53049(5)$ & $0.1098(2)$ & $0.39999(7)$ & $0.0282(4)$ \\
\hline C10' & $0.53990(5)$ & $0.2529(2)$ & $0.43641(7)$ & $0.0282(4)$ \\
\hline $\mathrm{H}^{\prime} \mathrm{O}^{\prime}$ & 0.5274 & 0.3523 & 0.4236 & 0.037 \\
\hline C11' & $0.56878(5)$ & $0.2225(2)$ & $0.49062(7)$ & $0.0230(3)$ \\
\hline C12' & $0.58228(6)$ & $0.3425(2)$ & $0.53989(7)$ & $0.0298(4)$ \\
\hline $\mathrm{H}_{12 \mathrm{a}^{\prime}}$ & 0.5653 & 0.3176 & 0.5679 & 0.040 \\
\hline $\mathrm{H} 12 \mathrm{~b}^{\prime}$ & 0.5715 & 0.4457 & 0.5231 & 0.040 \\
\hline $\mathrm{C}^{\prime \prime}{ }^{\prime}$ & $0.63455(6)$ & $0.3571(2)$ & $0.57477(7)$ & $0.0283(4)$ \\
\hline $\mathrm{H}^{\prime} 3^{\prime}$ & 0.6364 & 0.4391 & 0.6048 & 0.038 \\
\hline C14' & $0.65595(6)$ & $0.2070(2)$ & $0.60956(7)$ & $0.0308(4)$ \\
\hline $\mathrm{H} 14 \mathrm{a}^{\prime}$ & 0.6836 & 0.2379 & 0.6414 & 0.041 \\
\hline$H 14 b^{\prime}$ & 0.6334 & 0.1639 & 0.6278 & 0.041 \\
\hline C15' & $0.66269(6)$ & $0.4197(2)$ & $0.53603(7)$ & $0.0309(4)$ \\
\hline $\mathrm{C} 16^{\prime}$ & $0.73781(8)$ & $0.4917(3)$ & $0.53202(10)$ & $0.0648(7)$ \\
\hline H16a' & 0.7291 & 0.5989 & 0.5199 & 0.097 \\
\hline$H 16 b^{\prime}$ & 0.7344 & 0.4268 & 0.4976 & 0.097 \\
\hline H16 $c^{\prime}$ & 0.7699 & 0.4893 & 0.5566 & 0.097 \\
\hline O1' & $0.65528(4)$ & $0.0015(2)$ & $0.47351(5)$ & $0.0403(3)$ \\
\hline O2' & $0.50427(4)$ & $0.0978(2)$ & $0.34930(5)$ & $0.0429(3)$ \\
\hline $\mathrm{O}^{\prime}$ & $0.64645(5)$ & $0.4609(2)$ & $0.48563(6)$ & $0.0614(5)$ \\
\hline O4' & $0.70777(5)$ & $0.4317(2)$ & $0.56523(6)$ & $0.0584(5)$ \\
\hline \multicolumn{5}{|c|}{$U_{e q}=1 / 3\left[U_{11}\left(a a^{*}\right)^{2}+U_{22}\left(b b^{*}\right)^{2}+U_{33}\left(c c^{*}\right)^{2}+2 U_{12} a a^{*} b b^{*} \cos \gamma+2 U_{13} a a^{*} c c^{*} \cos \beta+2 U_{23} b^{*} c c^{*} \cos \alpha\right]$} \\
\hline
\end{tabular}


Table 3. Refined Thermal Parameters (U's) for Compound 2033

\begin{tabular}{|c|c|c|c|c|c|c|}
\hline Atom & $U_{11}$ & $\mathrm{U}_{22}$ & $\mathrm{U}_{33}$ & $\mathrm{U}_{23}$ & $U_{13}$ & $\mathrm{U}_{12}$ \\
\hline $\mathrm{C} 1$ & $0.0239(7)$ & $0.0166(7)$ & $0.0214(7)$ & $-0.0011(6)$ & $0.0097(6)$ & $-0.0019(6)$ \\
\hline $\mathrm{C} 2$ & $0.0251(8)$ & $0.0300(9)$ & $0.0235(8)$ & $-0.0026(7)$ & $0.0061(6)$ & $-0.0018(6)$ \\
\hline C3 & $0.0424(10)$ & $0.0362(10)$ & $0.0338(9)$ & $-0.0059(8)$ & $0.0035(8)$ & $-0.0125(8)$ \\
\hline C4 & $0.0697(13)$ & $0.0264(9)$ & $0.0341(9)$ & $-0.0089(8)$ & $0.0186(9)$ & $-0.0091(9)$ \\
\hline C5 & $0.0543(11)$ & $0.0241(9)$ & $0.0442(10)$ & $-0.0022(8)$ & $0.0279(9)$ & $0.0050(8)$ \\
\hline C6 & $0.0332(9)$ & $0.0270(9)$ & $0.0405(9)$ & $0.0047(7)$ & $0.0222(7)$ & $0.0067(7)$ \\
\hline $\mathrm{C} 7$ & $0.0209(7)$ & $0.0230(8)$ & $0.0249(8)$ & $0.0048(6)$ & $0.0093(6)$ & $0.0003(6)$ \\
\hline C8 & $0.0239(8)$ & $0.0289(9)$ & $0.0318(8)$ & $0.0089(7)$ & $0.0055(7)$ & $0.0000(6)$ \\
\hline $\mathrm{C9}$ & $0.0221(7)$ & $0.0310(9)$ & $0.0305(8)$ & $0.0004(7)$ & $0.0093(6)$ & $0.0018(7)$ \\
\hline $\mathrm{C}_{10}$ & $0.0247(8)$ & $0.0243(8)$ & $0.0365(9)$ & $0.0015(7)$ & $0.0119(7)$ & $-0.0020(6)$ \\
\hline C11 & $0.0209(7)$ & $0.0225(8)$ & $0.0312(8)$ & $0.0041(6)$ & $0.0142(6)$ & $0.0023(6)$ \\
\hline $\mathrm{C} 12$ & $0.0315(8)$ & $0.0233(8)$ & $0.0322(8)$ & $0.0065(7)$ & $0.0176(7)$ & $0.0023(6)$ \\
\hline $\mathrm{C}_{13}$ & $0.0329(8)$ & $0.0261(8)$ & $0.0227(8)$ & $0.0072(6)$ & $0.0126(6)$ & $0.0067(7)$ \\
\hline $\mathrm{C} 14$ & $0.0357(9)$ & $0.0326(9)$ & $0.0201(7)$ & $0.0006(7)$ & $0.0067(6)$ & $0.0063(7)$ \\
\hline C15 & $0.0322(8)$ & $0.0212(8)$ & $0.0278(8)$ & $0.0060(7)$ & $0.0132(7)$ & $0.0048(6)$ \\
\hline C16 & $0.0397(10)$ & $0.0532(12)$ & $0.0559(12)$ & $0.0051(10)$ & $0.0291(9)$ & $0.0141(9)$ \\
\hline 01 & $0.0300(6)$ & $0.0398(7)$ & $0.0247(6)$ & $0.0004(5)$ & $0.0150(5)$ & $-0.0032(5)$ \\
\hline $\mathrm{O} 2$ & $0.0373(7)$ & $0.0407(7)$ & $0.0348(7)$ & $0.0007(6)$ & $-0.0011(5)$ & $-0.0004(6)$ \\
\hline $\mathrm{O} 3$ & $0.0418(7)$ & $0.0513(8)$ & $0.0339(7)$ & $-0.0111(6)$ & $0.0138(6)$ & $0.0071(6)$ \\
\hline O4 & $0.0301(6)$ & $0.0544(8)$ & $0.0384(7)$ & $0.0002(6)$ & $0.0137(5)$ & $0.0142(6)$ \\
\hline$C 1^{\prime}$ & $0.0251(8)$ & $0.0228(8)$ & $0.0273(8)$ & $0.0010(6)$ & $0.0107(6)$ & $-0.0017(6)$ \\
\hline $\mathrm{C} 2^{\prime}$ & $0.0237(8)$ & $0.0380(10)$ & $0.0299(8)$ & $0.0016(7)$ & $0.0054(6)$ & $-0.0042(7)$ \\
\hline C3' & $0.0374(10)$ & $0.0416(11)$ & $0.0424(10)$ & $0.0059(9)$ & $-0.0027(8)$ & $0.0028(8)$ \\
\hline $\mathrm{C} 4^{\prime}$ & $0.0535(12)$ & $0.0335(10)$ & $0.0351(10)$ & $0.0122(8)$ & $0.0037(8)$ & $0.0001(9)$ \\
\hline $\mathrm{C}^{\prime}$ & $0.0426(10)$ & $0.0277(9)$ & $0.0389(10)$ & $0.0069(8)$ & $0.0128(8)$ & $-0.0068(8)$ \\
\hline$C 6^{\prime}$ & $0.0260(8)$ & $0.0301(9)$ & $0.0326(8)$ & $0.0020(7)$ & $0.0116(7)$ & $-0.0056(7)$ \\
\hline C7' & $0.0224(8)$ & $0.0243(8)$ & $0.0218(7)$ & $-0.0022(6)$ & $0.0067(6)$ & $-0.0017(6)$ \\
\hline $\mathrm{C}^{\prime}$ & $0.0300(8)$ & $0.0289(9)$ & $0.0273(8)$ & $-0.0059(7)$ & $0.0042(7)$ & $-0.0021(7)$ \\
\hline $\mathrm{Cg}{ }^{\prime}$ & $0.0242(8)$ & $0.0335(9)$ & $0.0260(8)$ & $0.0000(7)$ & $0.0061(6)$ & $-0.0080(7)$ \\
\hline C10' & $0.0269(8)$ & $0.0258(8)$ & $0.0311(8)$ & $0.0037(7)$ & $0.0076(7)$ & $-0.0014(7)$ \\
\hline $\mathrm{C}_{11}$ & $0.0212(7)$ & $0.0239(8)$ & $0.0264(8)$ & $-0.0023(6)$ & $0.0110(6)$ & $-0.0039(6)$ \\
\hline $\mathrm{C} 12^{\prime}$ & $0.0347(9)$ & $0.0276(9)$ & $0.0304(8)$ & $-0.0057(7)$ & $0.0145(7)$ & $-0.0020(7)$ \\
\hline $\mathrm{C} 13^{\prime}$ & $0.0372(9)$ & $0.0282(9)$ & $0.0212(7)$ & $-0.0064(7)$ & $0.0113(7)$ & $-0.0097(7)$ \\
\hline C14' & $0.0346(9)$ & $0.0367(9)$ & $0.0195(7)$ & $-0.0015(7)$ & $0.0057(6)$ & $-0.0115(7)$ \\
\hline $\mathrm{C} 15^{\prime}$ & $0.0405(9)$ & $0.0295(9)$ & $0.0232(8)$ & $-0.0063(7)$ & $0.0104(7)$ & $-0.0108(7)$ \\
\hline C16' & $0.0473(12)$ & $0.105(2)$ & $0.0461(12)$ & $0.0032(13)$ & $0.0210(10)$ & $-0.0335(13)$ \\
\hline O1' & $0.0335(7)$ & $0.0548(8)$ & $0.0393(7)$ & $-0.0127(6)$ & $0.0210(6)$ & $-0.0044(6)$ \\
\hline $\mathrm{O} 2^{\prime}$ & $0.0452(7)$ & $0.0450(8)$ & $0.0280(6)$ & $-0.0001(6)$ & $-0.0050(5)$ & $-0.0095(6)$ \\
\hline O3' & $0.0530(9)$ & $0.0972(13)$ & $0.0319(7)$ & $0.0174(8)$ & $0.0097(6)$ & $-0.0231(8)$ \\
\hline O4' & $0.0371(7)$ & $0.1035(13)$ & $0.0324(7)$ & $0.0087(8)$ & $0.0073(6)$ & $-0.0314(8)$ \\
\hline
\end{tabular}


Table 4. Bond Distances in Compound 2033, $\AA$

\begin{tabular}{|c|c|c|c|c|c|}
\hline $\mathrm{C} 1-01$ & $1.213(2)$ & C1-C2 & $1.519(2)$ & C1-C7 & $1.538(2)$ \\
\hline $\mathrm{C} 2-\mathrm{C}_{14}$ & $1.535(2)$ & $\mathrm{C} 2-\mathrm{C} 3$ & $1.546(2)$ & C3-C4 & $1.521(3)$ \\
\hline C4-C5 & $1.525(3)$ & C5-C6 & $1.534(2)$ & C6-C7. & $1.544(2)$ \\
\hline C7-C11 & $1.536(2)$ & $\mathrm{C} 7-\mathrm{C} 8$ & $1.542(2)$ & $\mathrm{C8}-\mathrm{C9}$ & $1.508(2)$ \\
\hline $\mathrm{C} 9-\mathrm{O} 2$ & $1.223(2)$ & C9-C10 & $1.455(2)$ & $\mathrm{C}_{10}-\mathrm{C}_{11}$ & $1.335(2)$ \\
\hline $\mathrm{C}_{11-\mathrm{C}_{12}}$ & $1.495(2)$ & $\mathrm{C} 12-\mathrm{C} 13$ & $1.539(2)$ & $\mathrm{C}_{13}-\mathrm{C}_{15}$ & $1.518(2)$ \\
\hline C13-C14 & $1.537(2)$ & C15-O3 & $1.197(2)$ & C15-O4 & $1.340(2)$ \\
\hline C16-O4 & $1.445(2)$ & C1'-O1' & $1.212(2)$ & $C 1^{\prime}-C_{2}^{\prime}$ & $1.523(2)$ \\
\hline$C 1^{\prime}-\mathrm{C} 7^{\prime}$ & $1.539(2)$ & C2'-C14' & $1.534(2)$ & $\mathrm{C}^{\prime}-\mathrm{C3}^{\prime}$ & $1.544(3)$ \\
\hline C3'-C4' & $1.527(3)$ & $\mathrm{C} 4^{\prime}-\mathrm{C} 5^{\prime}$ & $1.531(2)$ & $\mathrm{C} 5^{\prime}-\mathrm{C} 6^{\prime}$ & $1.530(2)$ \\
\hline $\mathrm{C} 6^{\prime}-\mathrm{C} 7^{\prime}$ & $1.549(2)$ & C7'-C11! & $1.535(2)$ & C7'-C8' & $1.540(2)$ \\
\hline C8'-C9' & $1.503(2)$ & $\mathrm{C} 9^{\prime}-\mathrm{O} 2^{\prime}$ & $1.221(2)$ & C9'-C10' & $1.458(2)$ \\
\hline C10'-C11' & $1.335(2)$ & C11'-C12' & $1.503(2)$ & $\mathrm{C}_{12}^{\prime}-\mathrm{C}_{13}{ }^{\prime}$ & $1.534(2)$ \\
\hline C13'-C15' & $1.510(2)$ & $\mathrm{C}_{13}^{\prime}-\mathrm{C} 14^{\prime}$ & $1.539(2)$ & $\mathrm{C} 15^{\prime}-\mathrm{O} 3^{\prime}$ & $1.193(2)$ \\
\hline $\mathrm{C} 15^{\prime}-\mathrm{O} 4^{\prime}$ & $1.318(2)$ & $\mathrm{C} 16^{\prime}-\mathrm{O} 4^{\prime}$ & $1.448(2)$ & & \\
\hline
\end{tabular}

Table 5. Bond Angles in Compound 2033,

\begin{tabular}{|c|c|c|c|c|c|}
\hline $\mathrm{O} 1-\mathrm{C}_{1}-\mathrm{C}_{2}$ & $118.25(13)$ & O1-C1-C7 & $120.62(13)$ & $\mathrm{C} 2-\mathrm{C} 1-\mathrm{C} 7$ & $121.12(12)$ \\
\hline $\mathrm{C}_{1}-\mathrm{C}_{2}-\mathrm{C}_{14}$ & $116.96(13)$ & $\mathrm{C}_{1}-\mathrm{C} 2-\mathrm{C} 3$ & $108.30(13)$ & $\mathrm{C}_{14-\mathrm{C} 2-\mathrm{C}_{3}}$ & $113.62(13)$ \\
\hline $\mathrm{C} 4-\mathrm{C} 3-\mathrm{C} 2$ & $116.3(2)$ & C3-C4-C5 & $115.60(14)$ & C4-C5-C6 & $113.9(2)$ \\
\hline $\mathrm{C} 5-\mathrm{C} 6-\mathrm{C} 7$ & $114.32(13)$ & $\mathrm{C} 11-\mathrm{C} 7-\mathrm{C} 1$ & $107.16(12)$ & $\mathrm{C} 11-\mathrm{C} 7-\mathrm{C} 8$ & $102.86(12)$ \\
\hline $\mathrm{C} 1-\mathrm{C} 7-\mathrm{C} 8$ & $110.73(12)$ & C11-C7-C6 & $112.05(12)$ & C1-C7-C6 & $111.85(12)$ \\
\hline $\mathrm{C} 8-\mathrm{C} 7-\mathrm{C} 6$ & $111.76(13)$ & C9-C8-C7 & $106.01(13)$ & $\mathrm{O} 2-\mathrm{C} 9-\mathrm{C}_{10}$ & $127.2(2)$ \\
\hline $\mathrm{O} 2-\mathrm{C} 9-\mathrm{C} 8$ & $125.0(2)$ & C10-C9-C8 & $107.82(13)$ & $C_{11-C}-C_{0}$ & $111.12(14)$ \\
\hline $\mathrm{C}_{10-\mathrm{C}_{11}-\mathrm{C}_{12}}$ & $124.98(14)$ & C10-C11-C7 & $112.00(13)$ & $\mathrm{C} 12-\mathrm{C}_{11-C 7}$ & $122.91(14)$ \\
\hline $\mathrm{C}_{11}-\mathrm{C}_{12}-\mathrm{C}_{13}$ & $118.01(12)$ & $\mathrm{C}_{15}-\mathrm{C}_{13}-\mathrm{C}_{14}$ & $111.21(13)$ & $\mathrm{C}_{15}-\mathrm{C}_{13}-\mathrm{C}_{12}$ & $111.69(13)$ \\
\hline $\mathrm{C}_{14}-\mathrm{C}_{13}-\mathrm{C}_{12}$ & $114.45(13)$ & $\mathrm{C}_{2}-\mathrm{C}_{14-\mathrm{C}_{13}}$ & $115.21(12)$ & $\mathrm{O} 3-\mathrm{C} 15-\mathrm{O} 4$ & $123.41(14)$ \\
\hline $\mathrm{O} 3-\mathrm{C}_{15}-\mathrm{C}_{13}$ & $125.9(2)$ & $\mathrm{O} 4-\mathrm{C} 15-\mathrm{C} 13$ & $110.71(13)$ & $\mathrm{C}_{15}-\mathrm{O} 4-\mathrm{C} 16$ & $116.06(14)$ \\
\hline $\mathrm{O} 1^{\prime}-\mathrm{C} 1^{\prime}-\mathrm{C} 2^{\prime}$ & $118.41(14)$ & $O 1^{\prime}-\mathrm{C} 1^{\prime}-\mathrm{C} 7^{\prime}$ & $120.62(14)$ & $\mathrm{C} 2{ }^{\prime}-\mathrm{C} 1^{\prime}-\mathrm{C} 7^{\prime}$ & $120.93(12)$ \\
\hline $\mathrm{C}_{1}^{\prime}-\mathrm{C}^{\prime}-\mathrm{C} 14^{\prime}$ & $118.01(13)$ & $C 1^{\prime}-C_{2}^{\prime}-{ }^{\prime} 3^{\prime}$ & $108.01(14)$ & $\mathrm{C}^{\prime} 4^{\prime}-\mathrm{C}^{\prime}-\mathrm{C}^{\prime}$ & $112.73(14)$ \\
\hline C4'-C3'-C2' & $116.6(2)$ & $\mathrm{C} 3^{\prime}-\mathrm{C} 4^{\prime}-\mathrm{C5}^{\prime}$ & $115.4(2)$ & $\mathrm{C} 6^{\prime}-\mathrm{C} 5^{\prime}-\mathrm{C} 4^{\prime}$ & $113.6(2)$ \\
\hline$C 5^{\prime}-C 6^{\prime}-C 7^{\prime}$ & $115.02(13)$ & $C 11^{\prime}-C 7^{\prime}-C_{1}^{\prime}$ & $108.60(12)$ & $C 11^{\prime}-C 7^{\prime}-C 8^{\prime}$ & $102.81(12)$ \\
\hline$C 1^{\prime}-C 7^{\prime}-C 8^{\prime}$ & $110.73(12)$ & $C 11^{\prime}-\mathrm{C} 7^{\prime}-\mathrm{C} 6^{\prime}$ & $111.80(12)$ & $C 1^{\prime}-C 7^{\prime}-C 6^{\prime}$ & $110.61(12)$ \\
\hline C8'-C7'-C6' & $112.01(13)$ & C9'-C8'-C7' & $106.34(13)$ & O2'-C9'-C10' & $126.8(2)$ \\
\hline O2'-C9'-C8' & $125.6(2)$ & C10'-C9'-C8' & $107.61(13)$ & $C 11^{\prime}-C_{10}^{\prime}-C 9^{\prime}$ & $111.13(14)$ \\
\hline$C_{10}^{\prime}-C_{11}^{\prime}-C_{12}^{\prime}$ & $123.9(2)$ & $\mathrm{C} 10^{\prime}-\mathrm{C} 11^{\prime}-\mathrm{C} 7^{\prime}$ & $111.96(13)$ & $C_{12}^{\prime}-C_{11}^{\prime}-C 7^{\prime}$ & $124.02(13)$ \\
\hline$C^{\prime} 1^{\prime}-C 12^{\prime}-C 13^{\prime}$ & $117.51(13)$ & $\mathrm{C}_{15}{ }^{\prime}-\mathrm{C} 13^{\prime}-\mathrm{C} 12^{\prime}$ & $111.32(13)$ & C15'-C13'-C14' & $113.15(14)$ \\
\hline C12'-C13'-C14' & $114.39(13)$ & $C 2^{\prime}-\mathrm{C} 14^{\prime}-\mathrm{C} 13^{\prime}$ & $116.37(13)$ & $\mathrm{O} 3^{\prime}-\mathrm{C} 15^{\prime}-\mathrm{O} 4^{\prime}$ & $122.9(2)$ \\
\hline $\mathrm{O} 3^{\prime}-\mathrm{C} 15^{\prime}-\mathrm{C} 13^{\prime}$ & $124.9(2)$ & $\mathrm{O}^{\prime}-\mathrm{C} 15^{\prime}-\mathrm{C} 13^{\prime}$ & $112.08(14)$ & $\mathrm{C}^{\prime} 5^{\prime}-\mathrm{O} 4^{\prime}-\mathrm{C} 16^{\prime}$ & $116.4(2)$ \\
\hline
\end{tabular}

